Wright State University

CORE Scholar

Physics Faculty Publications

Physics

$10-1-2001$

\title{
Solar Activity Variations of the Venus Thermosphere/lonosphere
}

Jane L. Fox

Wright State University - Main Campus, jane.fox@wright.edu

K. Y. Sung

Follow this and additional works at: https://corescholar.libraries.wright.edu/physics

Part of the Physics Commons

\section{Repository Citation}

Fox, J. L., \& Sung, K. Y. (2001). Solar Activity Variations of the Venus Thermosphere/lonosphere. Journal of Geophysical Research-Space Physics, 106 (A10), 21305-21335.

https://corescholar.libraries.wright.edu/physics/17

This Article is brought to you for free and open access by the Physics at CORE Scholar. It has been accepted for inclusion in Physics Faculty Publications by an authorized administrator of CORE Scholar. For more information, please contact library-corescholar@wright.edu. 


\title{
Solar activity variations of the Venus thermosphere/ionosphere
}

\author{
J. L. Fox ${ }^{1}$ and K. Y. Sung \\ Institute for Terrestrial and Planetary Atmospheres, State University of New York at Stony \\ Brook, New York, USA
}

\begin{abstract}
We present models of the low and high solar activity thermospheres and ionospheres of Venus for a background atmosphere based largely on the VTS3 model of Hedin et al. [1983]. Our background model consists of 12 neutral species, and we compute the density profiles of 13 ions and 7 minor neutrals. We find that the peak production rates of some ions, such as $\mathrm{CO}_{2}^{+}$and $\mathrm{N}_{2}^{+}$, vary approximately as the solar flux and that some, whose parent neutrals are photochemically produced, such as $\mathrm{O}^{+}, \mathrm{N}^{+}$, and $\mathrm{C}^{+}$, show variations that are amplified over that of the solar flux. The solar cycle variation of the $\mathrm{O}_{2}^{+}$density at its peak is about a factor of 1.6 , in good agreement with the radio occultation measurements and previous models. The peak density of $\mathrm{N}_{2}^{+}$varies by a factor of $\sim 2$, but that of $\mathrm{CO}^{+}$varies by a larger factor because the mixing ratio of $\mathrm{CO}$ is also correlated with solar activity. The atomic ions $\mathrm{O}^{+}, \mathrm{N}^{+}$, and $\mathrm{C}^{+}$, which peak at high altitudes, exhibit larger density enhancements at high solar activity of factors of 5-18. Thus there is a solar cycle variation in the overall composition of the ionosphere, with a relatively larger proportion of atomic ions at high solar activity. In both measurements and models the solar activity variation of the electron density profile is altitude-dependent, with variations of only $\sim 60 \%$ near the peak but up to an order of magnitude near $300 \mathrm{~km}$. The high solar activity electron density profile exhibits an $F_{2}$ "shoulder," which is rarely seen in the radio occultation data and may indicate that the VTS3 atomic $\mathrm{O}$ mixing ratios are too large at high solar activity. We also discuss the solar activity variations of $\mathrm{N}, \mathrm{NO}, \mathrm{C}, \mathrm{O}\left({ }^{1} D\right)$, and $\mathrm{O}\left({ }^{1} S\right)$.
\end{abstract}

\section{Introduction}

The atmosphere of Venus is composed mainly of $\mathrm{CO}_{2}$ and $\mathrm{N}_{2}$; the thermosphere also exhibits significant densities of the photolysis products $\mathrm{O}, \mathrm{CO}, \mathrm{C}$, and $\mathrm{N}$, the photochemically produced species $\mathrm{O}_{2}$ and $\mathrm{NO}$, and the light species $\mathrm{H}_{2}, \mathrm{H}$, and $\mathrm{He}$. In the lower thermosphere, $\mathrm{CO}_{2}$ is the major constituent, but $\mathrm{O}$ becomes more important above altitudes of $150-160 \mathrm{~km}$. The major difference between the thermospheres of Mars and Venus is the greater abundance of $\mathrm{O}$ and $\mathrm{CO}$ on Venus, presumably owing to its closer proximity to the Sun. In addition, Ar is much less abundant on Venus than on Earth or Mars.

The electron density profile in the Venus ionosphere was first measured by the radio occultation experiment on Mariner 5 [Kliore et al., 1967] and by many subsequent spacecraft over the period 1974-1992 (see, for

${ }^{1}$ Also at Department of Physics, Wright State University, Dayton, Ohio, USA.

Copyright 2001 by the American Geophysical Union.

Paper number 2001JA000069.

0148-0227/01/2001JA000069\$09.00 example, the introduction in the paper by Kliore and Luhmann [1991]). The ionosphere that results from solar photoionization and photoelectron impact ionization has been modeled by many investigators over the last 23 years [e.g., Chen and Nagy, 1978; Nagy et al., 1980; Cravens et al., 1981; Fox and Dalgarno, 1981; Fox and Victor, 1981; Fox, 1982a, 1982b; Kim et al., 1989; Fox and Kliore, 1997]. The in situ and remote-sensing measurements made by instruments on the Pioneer Venus (PV) spacecraft, which orbited the planet from 1978 to 1992, have provided the most detailed description of the Venus thermosphere/ionosphere at high solar activity. Comparable in situ measurements at low solar activity are lacking, since periapsis of the PV spacecraft was outside the ionosphere during solar minimum periods. Measurements were carried out, however, by the PV Orbiter Radio Occultation (ORO) experiment [e.g, Kliore et al., 1979] at all phases of the solar cycle during the nearly 14-year life of the spacecraft. For a brief period of moderately low solar activity $\left(F_{10} \approx \approx 120\right)$ in 1992, the PV spacecraft carried out in situ measurements, which were mostly in the predawn sector, as it reentered the atmosphere.

A summary of models of the Venus ionosphere before 1983 has been presented by Nagy et al. [1983]. Nagy 
et al. [1980] modeled the density profiles of seven ions including both chemistry and transport and of three additional ions that were assumed to be in photochemical equilibrium. They compared their profiles to the in situ measurements of the PV Orbiter Ion Mass Spectrometer (OIMS) [e.g., Taylor et al., 1980]. The dayside peak densities of $\mathrm{O}_{2}^{+}$measured by the OIMS were near $170 \mathrm{~km}, \sim 30 \mathrm{~km}$ higher than the radio occultation measurements indicated, and are probably anomalous. Nevertheless, the other ion density profiles modeled by Nagy et al. [1980] showed good agreement, except for the mass-28 ions $\left(\mathrm{CO}^{+}\right.$and $\left.\mathrm{N}_{2}^{+}\right), \mathrm{N}^{+}$, and $\mathrm{C}^{+}$. These discrepancies were mostly resolved by $F_{0 x}$ [1982a], who showed that the chemistry of metastable species $\mathrm{O}^{+}\left({ }^{2} D\right), \mathrm{O}^{+}\left({ }^{2} P\right), \mathrm{N}\left({ }^{2} D\right)$, and $\mathrm{N}\left({ }^{2} P\right)$ is important in the production of $\mathrm{N}^{+}, \mathrm{CO}^{+}$, and $\mathrm{N}_{2}^{+}$. Fox [1982b] also showed that the $\mathrm{C}^{+}$profile could be reproduced when photoionization of ambient $\mathrm{C}$ was taken into account.

Cravens et al. [1981] modeled the ionosphere below $170 \mathrm{~km}$ as a function of solar zenith angle and for various assumptions about the electron temperature and the underlying neutral atmosphere. They compared their model electron density profiles with those measured by the PV ORO profiles during the first three seasons, in which the average values of $F_{10} 7$ were in the range 175-215. The peak densities from both the high solar activity PV measurements and the Venera 9 and 10 radio occultation profiles [e.g., Aleksandrov et al., 1976], which were obtained in 1975 at low solar activity, were also compared to those computed from Chapman theory [Chapman, 1931]. They showed that the solar zenith angle dependence of the peak electron density was well reproduced by Chapman theory and by their models. In contrast to Chapman theory, however, which predicts that the electron density peak rises as the solar zenith angle (SZA) increases, the altitude of the measured peak was shown to remain near $140 \mathrm{~km}$ from $0^{\circ}$ to $70^{\circ}$ SZA at high solar activity. This was explained as being due to the collapse of the neutral atmosphere, which compensates for the increase in path length as the solar zenith angle becomes larger. Cravens et al. also noted the occurrence of a ledge in the electron density profile near $125 \mathrm{~km}$, which they attributed to the absorption of soft $\mathrm{X}$ rays.

Kim et al. [1989] computed density profiles of six ions below $180 \mathrm{~km}$, including $\mathrm{O}_{2}^{+}, \mathrm{O}^{+}, \mathrm{CO}_{2}^{+}, \mathrm{NO}^{+}, \mathrm{H}^{+}$, and $\mathrm{CO}^{+}$, for low and high solar activity photochemical equilibrium models, and they compared their results to averages of $16 \mathrm{PV}$ ORO profiles during solar maximum and 11 ORO profiles during solar minimum. Knudsen [1992] reviewed the structure, energetics, and solar cycle variability of the dayside and nightside ionospheres based on measurements from the first 12 years of the $\mathrm{Pi}$ oneer Venus spacecraft. Dalgarno and Fox [1994] have presented a comprehensive review of ion chemistry in planetary atmospheres (including that of Venus) and astrophysical environments.
Many rate coefficients for relevant ion-molecule, neutral-neutral, and dissociative recombination reactions have been revised in the 10-15 years since most of the models of the ionosphere of Venus were constructed. Using updated values for the chemical rate coefficients and cross sections for photon and electron interactions with the background atmosphere, we model here the density profiles of 13 ions and 7 minor neutral species, with an emphasis on their variations with solar activity. A preliminary version of this model was presented briefly by Fox and Kliore [1997]. We compare the results to previous models and to the available in situ and radio occultation data.

\section{The Model}

Density profiles for $\mathrm{CO}_{2}, \mathrm{~N}_{2}, \mathrm{O}, \mathrm{CO}, \mathrm{N}$, and $\mathrm{He}$ and the neutral temperature profiles were taken from the VTS3 model of Hedin et al. [1983], which is based on measurements of the PV Orbiter Neutral Mass Spectrometer (ONMS) [e.g., Niemann et al., 1980], normalized to the Orbiter Atmospheric Drag (OAD) data [e.g., Keating et al., 1980]. An $\mathrm{O}_{2}$ mixing ratio of $3 \times 10^{-3}$ was adopted, as inferred by Paxton [1985] from the atomic carbon density profiles derived from the intensities of the C I 1657- and 1561- $\AA$ airglow features measured by the PV Orbiter Ultraviolet Spectrometer (OUVS) [e.g., Stewart et al., 1979]. Eddy diffusion coefficients of $9 \times 10^{12} / n^{0.5} \mathrm{~cm}^{2} \mathrm{~s}^{-1}$ at high solar activity and $7 \times 10^{12} / \mathrm{n}^{05} \mathrm{~cm}^{2} \mathrm{~s}^{-1}$ at low solar activity, where $n$ is the total number density, were determined by one-dimensional fits to the VTS $3 \mathrm{~N}_{2}$ profiles and used to construct the density profiles of $\mathrm{O}_{2}, \mathrm{H}, \mathrm{H}_{2}$, $\mathrm{Ar}, \mathrm{NO}, \mathrm{N}$, and $\mathrm{C}$. The eddy diffusion coefficient in a one-dimensional model represents a combination of mixing due to large-scale winds and small-scale turbulence. One might expect the eddy diffusion coefficient to be larger at high solar activity than at low because the larger solar EUV fluxes at high solar activity lead to stronger thermospheric winds. Our derived values are, however, empirical and not based on any assumptions about the cause of the increase. The eddy diffusion coefficient derived from the PV Bus Neutral Mass Spectrometer measurements was within a factor of 2 of $1.4 \times 10^{13} / n^{1 / 2}$ [von Zahn et al., 1980] and is therefore in acceptable agreement with our high solar activity value.

There is a large range in the measured values for the mixing ratios for the isotopes of $\mathrm{Ar}$ in the atmosphere of Venus. The total mixing ratio of ${ }^{36} \mathrm{Ar}+{ }^{38} \mathrm{Ar}+$ ${ }^{40} \mathrm{Ar}$ was measured by several instruments on American and Soviet spacecraft. Values from 40 to $200 \mathrm{ppm}$ were recorded, and the Venus International Reference Atmosphere recommended a mixing ratio of $70 \pm 25 \mathrm{ppm}$ [von Zahn and Moroz, 1985]. Von Zahn et al. [1983] have argued that the gas chromatographic measurements are more reliable than the mass spectrometer measurements, since they are less subject to pumping problems and impurity leaks. We have chosen a value 
of $60 \mathrm{ppm}$ for total Ar, and we assumed that $\sim 55 \%$ is in the form of ${ }^{40} \mathrm{Ar}$ [von Zahn et al., 1983]. Thus the ${ }^{40} \mathrm{Ar}$ mixing ratio at the bottom boundary was assumed to be $33 \mathrm{ppm}$. Since the ${ }^{40} \mathrm{Ar}$ mixing ratio is so small, the effects of the error associated with this assumption and of the neglect of the other isotopes of Ar are insignificant for our model.

The solar maximum $\mathrm{H}$ density profiles were constructed by varying the bottom boundary condition so that the densities in the $160-$ to $180-\mathrm{km}$ region reproduced those derived by Taylor et al. [1984] [see also Brinton et al., 1980] from simultaneous measurements of $\mathrm{H}^{+}, \mathrm{O}^{+}$, and $\mathrm{O}$. Because the escape rates for $\mathrm{H}$ are larger at high solar activity, the $\mathrm{H}$ densities are anticorrelated with solar flux [e.g., Hartle et al., 1996; Donahue et al., 1997; Grebowsky et al., 1995]. Using the same method in the predawn bulge region during the reentry period of the PV spacecraft, Grebowsky et al. [1995] showed that the $\mathrm{H}$ densities were larger by a factor of $\sim 6$ at moderately low solar activity. We have assumed that the $\mathrm{H}$ densities are $\sim 6 \times 10^{5} \mathrm{~cm}^{-3}$ near $160 \mathrm{~km}$ in the low solar activity model and $\sim 1 \times 10^{5} \mathrm{~cm}^{-3}$ in the high solar activity model. The $\mathrm{H}_{2}$ mixing ratio of $1 \times 10^{-7}$ at the bottom boundary was adopted from the model of Yung and DeMore [1982].

The lower boundary of both models was assumed to be at $90 \mathrm{~km}$, and the upper boundary of the high solar activity model was assumed to be at $400 \mathrm{~km}$. Because the ionopause at solar minimum is observed to be lower than that of the high solar activity model, usually between 200 and $300 \mathrm{~km}$ [e.g., Kliore and Luhmann, 1991], the upper boundary of the low solar activity model was taken to be $320 \mathrm{~km}$.

For the VTS3 model, $F_{10.7}$ was assumed to be 80 at low solar activity and 200 at high solar activity, at equatorial latitudes, and 1600 hours local time; the solar zenith angle was therefore $60^{\circ}$. Figures $1 \mathrm{a}$ and $1 \mathrm{~b}$ show the background model atmospheres for 10 of the 12 species for low and high solar activities, respectively. The density profiles of NO and $\mathrm{C}$ were computed selfconsistently in the model and will be presented in section 5.2. Although $\mathrm{N}$ density profiles are given by the VTS3 model of Hedin et al. [1983], we also compute those densities and compare them to the VTS3 values.

The solar fluxes longward of $18 \AA$ were taken from the SC\#21REFW and F79050N spectra of H. Hinteregger (private communication, 1979) [see also Torr et al., 1979], for low and high solar activities, respectively. In these spectra the continuum fluxes are given at $1-\AA$ resolution, and the fluxes in the strong solar lines are given as integrated fluxes at the individual wavelengths. For wavelengths in the $0.5-18 \AA$ range the high solar activity fluxes in 1- $\AA$ bins were taken from the model of Ayres [1997, and private communication, 1997]. Solar activity variations increase with decreasing wavelength below $\sim 2000 \AA$, where they are in the $10-20 \%$ range, to factors of $2-4$ in the $500-1500 \AA$ region. At shorter wavelengths the variation in the solar fluxes exhibits considerable structure, and the variability in the shortwavelength solar lines can reach factors of $\sim 100$. In the harder X-ray region the variations are larger, reaching factors of $10^{3}-10^{5}$ for solar flares. Here we have somewhat arbitrarily assumed that the fluxes shortward of $18 \AA$ are a factor of 10 smaller at low solar activity than at high solar activity. This assumption may be an underestimate, and indeed, the actual variability may be due to the presence or absence of solar flares, but these short $X$ rays only affect the ion densities below $100 \mathrm{~km}$, where they are small, and the odd nitrogen densities in that altitude range. We have included photoionization, photodissociation (for molecules), and electron impact excitation, ionization, and dissociation (for molecules) of $\mathrm{CO}_{2}, \mathrm{Ar}, \mathrm{N}_{2}, \mathrm{O}, \mathrm{CO}, \mathrm{O}_{2}, \mathrm{NO}, \mathrm{N}, \mathrm{C}, \mathrm{H}, \mathrm{H}_{2}$, and He.

The ion and electron temperatures were adopted from PV measurements at high solar activity [e.g., Knudsen et al., 1979; Cravens et al., 1980], and the electron temperature in the low solar activity model was assumed to be the same as that at high solar activity. The electron temperatures are not expected to vary much with solar activity, because both the production mechanism for energetic electrons, photoionization, and the major energy loss process, collisions with ambient ions, increase with solar activity [e.g., Nagy and Cravens, 1997]. Brace et al. [1987] showed that in the 300-400 $\mathrm{km}$ region in the terrestrial ionosphere, $T_{e}$ exhibited a weak variation with $F_{10} 7$, increasing by $\sim 3 \mathrm{~K} / F_{10} 7$ unit for the $40^{\circ}-50^{\circ}$ latitude range, although at latitudes below $10^{\circ}$ the variation was $\sim 7 \mathrm{~K} / F_{10} 7$ unit. Electron temperature profiles on the Earth are not strictly comparable to those on Venus. On the unmagnetized planets, Venus and Mars, a heat source at the upper boundary of the models is required to reproduce the measured $T_{i}$ and $T_{e}$ profiles [e.g., Johnson, 1978; Cravens et al., 1980; Nagy et al., 1983; Nagy and Cravens, 1997; Brace and Kliore, 1991].

A study by Elphic et al. [1984] indicated that electron temperatures below $200 \mathrm{~km}$ in the daytime Venus ionosphere were not correlated with the 27-day variation in the solar fluxes. Thers and Brace [1993] showed that the nighttime electron temperatures above $\mathbf{1 7 0}$ km obtained by the PV Orbiter Electron Temperature Probe (OETP) [e.g., Brace et al., 1980] during the reentry period of the spacecraft, when the solar activity was moderately low $\left(F_{10} \approx 120\right)$, were slightly higher than those at high solar activity. The increase is consistent with the inverse correlation between electron densities and temperatures that has been reported by Knudsen et al. [1979], Dobe et al. [1993], and Mahajan et al. [1994]. Brace and Thers [1995] presented data from high-altitude measurements of the PV OETP during the reentry period of the PVO spacecraft showing that $T_{e}$ was either unchanged or slightly higher at moderately low solar activity compared to solar maximum. This result contradicted the suggestion of Kliore and Mullen [1989] that a $25 \%$ increase in $T_{e}$ was required to reproduce the solar cycle variation of the electron den- 


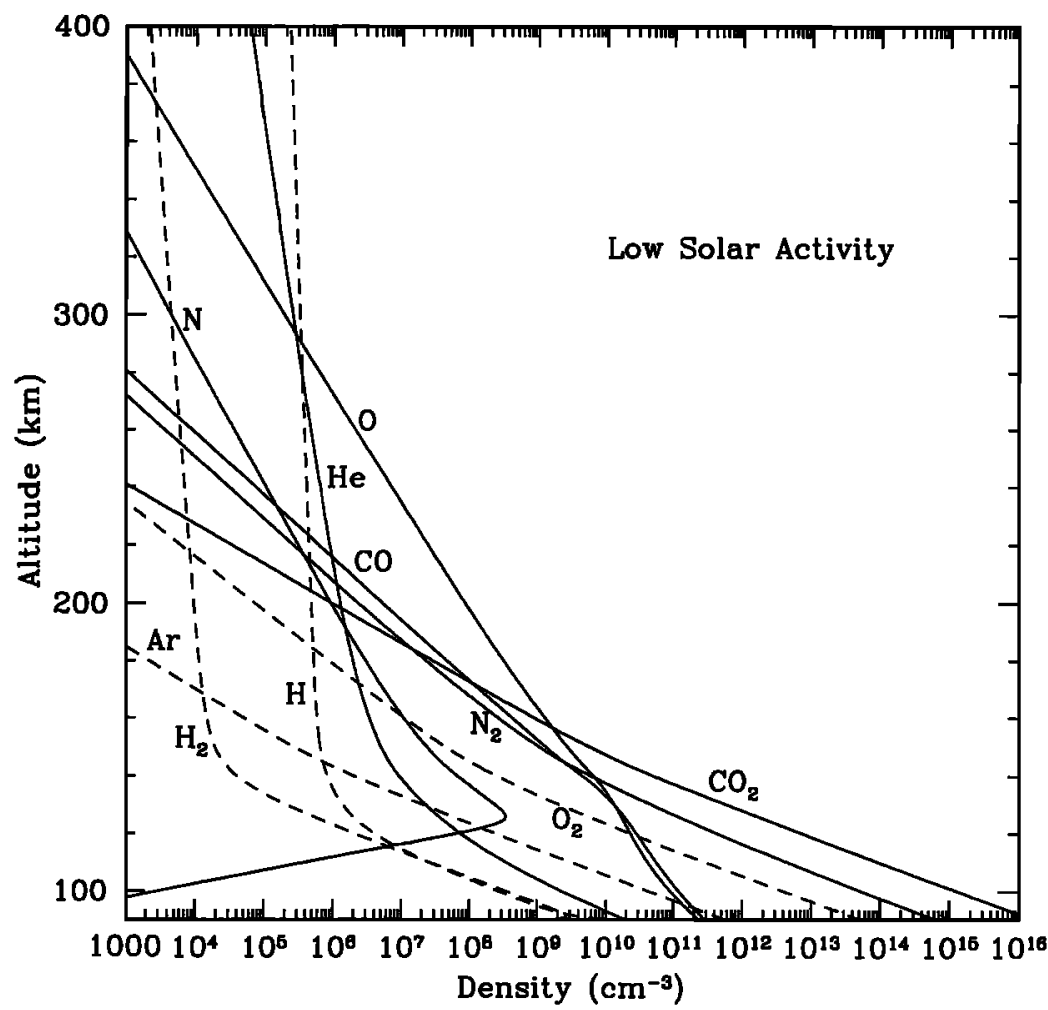

Figure 1a. Altitude profiles of neutral densities in the low solar activity model. The density profiles of species that are taken from the VTS3 model of Hedin et al. [1983] for $F_{10.7}=80$ are plotted with solid curves. The density profiles of the other species are shown as dashed curves and were constructed for the model (see text).

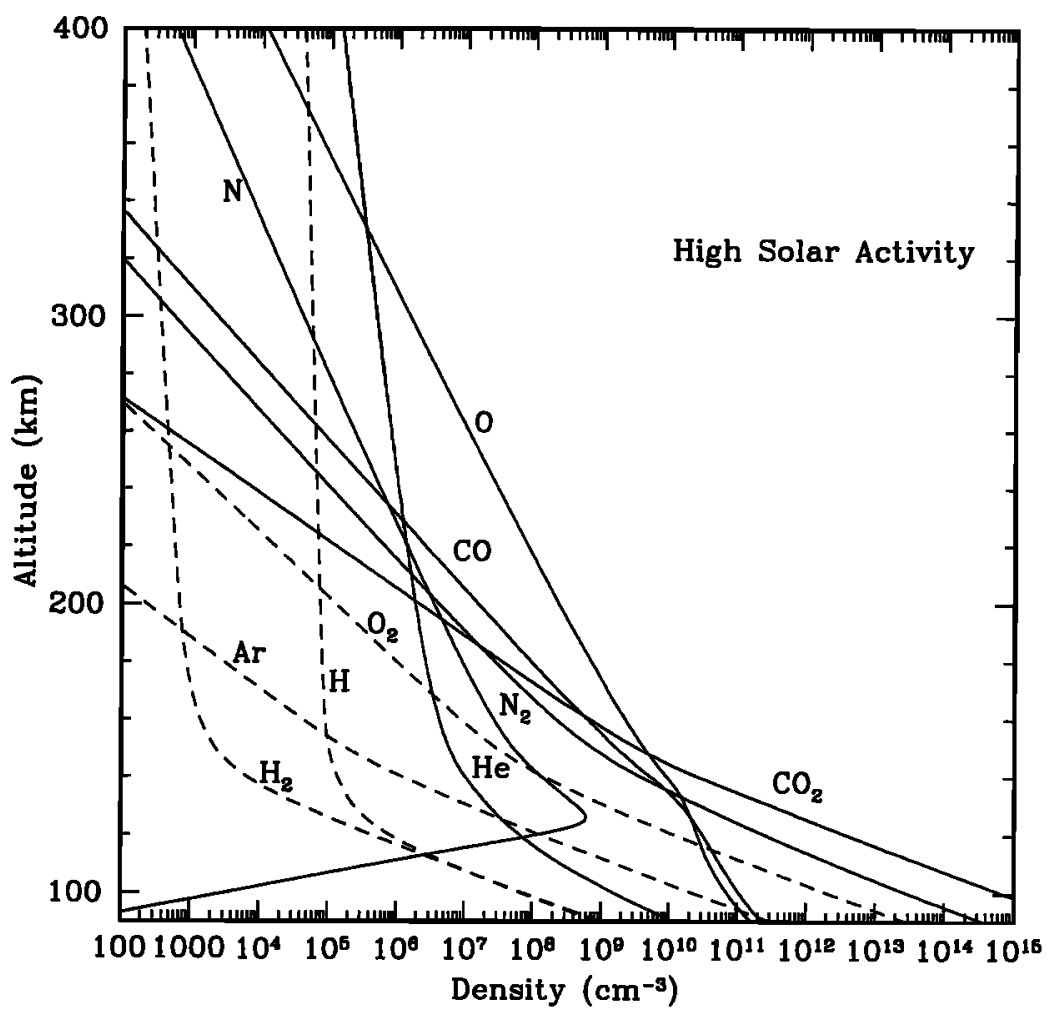

Figure 1b. Altitude profiles of neutral densities in the high solar activity model. The density profiles of species that are taken from the VTS3 model of Hedin et al. [1983] for $F_{10}=200$ are plotted with solid curves. The density profiles of the other species are shown as dashed curves and were constructed for the model (see text). 


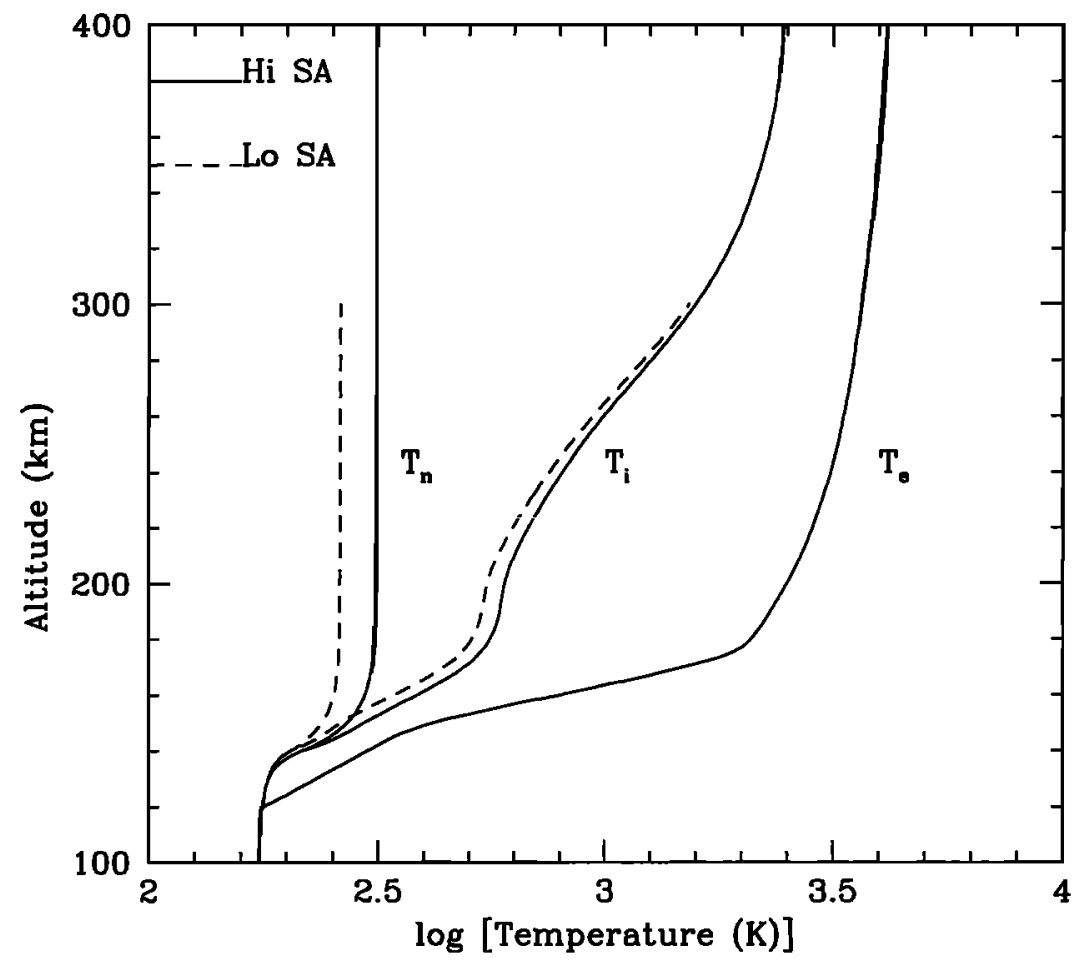

Figure 2. Altitude profiles for the neutral $\left(T_{n}\right)$, ion $\left(T_{i}\right)$, and electron $\left(T_{e}\right)$ temperatures adopted in the model. The solid curves are the high solar activity values, and the dashed curves are the low solar activity values. The electron temperatures were assumed to be the same for both models.

sity peak. We find here no need to vary $T_{e}$ to reproduce acceptably the values of the electron density peaks at high and low solar activities. It should, however, be noted that the altitude and magnitude of the electron density peak are strongly affected if the $T_{e}$ profile is assumed to diverge from the neutral temperature profile at or below the peak in the ion production rates.

Since there are no measurements of $T_{i}$ at solar minimum, the low solar activity ion temperatures were assumed to be only slightly smaller than those at high solar activity, and the difference was arbitrarily assumed to be equal to the difference between the low and high solar activity neutral temperatures. Because the ion temperatures are much larger than the neutral temperatures, the resulting fractional change in the ion temperatures is small at high altitudes. The neutral, ion, and electron temperature profiles adopted in the models for low and high solar activities are shown in Figure 2 .

\section{Cross Sections and Rate Coefficients}

The electron impact cross sections are similar to those used in our previous models of planetary atmospheres [see, e.g., Fox, 1993; Fox et al., 1996; Kim and Fox, 1994; Perry et al., 1999, and references therein]. Our complete compilation of electron impact cross sections has been presented recently by Sung and Fox [2000] and will be submitted for publication soon (K. Y. Sung and J. L. Fox, manuscript in preparation, 2001).
The cross sections for photoabsorption, photoionization, and (for molecules) photodissociation are similar to those we have used in our previous models. Only the major changes are described here. We have extended all the cross sections from the shortest wavelength measured to $0.5 \AA$ using the photoabsorption cross sections for atoms presented by Verner and Yakovlev [1995] and Verner et al. [1996]. The molecular cross sections at these short wavelengths are assumed to be the sum of the cross sections for the constituent atoms. The $\mathrm{CO}_{2}$ cross sections for photoabsorption in the wavelength range 900-1200 $\AA$ were taken from Chan et al. [1993a] (also C. Brion, Web site: ftp://chem.ubc.ca/pub/cooper), and photoabsorption, photoionization, and photodissociation cross sections in the range 490-900 $\AA$ were taken from Shaw et al. [1995]. In the region longward of $1200 \AA$ we adopted the photoabsorption cross sections of Lewis and Carver [1983] (also B. Lewis, private communication, 1994) for a temperature of 200 $\mathrm{K}$. The $\mathrm{N}_{2}$ cross sections are taken from Chan et al., [1993b] (also, C. Brion, Web site given above); we adopted the low-resolution cross sections up to $541 \AA$ and the high-resolution cross sections longward of that wavelength. The $\mathrm{O}_{2}$ photoabsorption cross sections are from Chan et al. [1993c] (also, C. E. Brion, Web site given above) to $413 \AA$. For absorption in the $\mathrm{O}_{2}$ Schumann Runge bands the high-resolution cross sections from the Center for Astrophysics Web site (cfawww.harvard.edu/amdata/ampdata/cfamols.htm) [see also, Yoshino et al., 1992] were averaged over 1- $\AA$ inter- 
vals in the continuum. The atomic $O$ photoabsorption cross sections were adopted from Samson and Pareek [1985] and Angel and Samson [1988]. The He photoabsorption cross sections were taken from Samson et al. [1994]. For $\mathrm{H}_{2}$ the high-resolution cross sections constructed by Kim and Fox [1994] were adopted for the solar lines and were averaged over $1-\AA$ intervals for the solar continuum. For atomic nitrogen from threshold to $31 \AA$ the photoabsorption cross sections of Samson and Angel [1990] were adopted, with the structure in the $610-700 \AA$ region averaged out.

The rate coefficients for ion-molecule, neutral-neutral, and dissociative recombination reactions have been extensively revised over the last decade, and complete lists are given in Tables 1, 2, and 3, respectively. In Tables $1-3$, the original sources are cited, except when an averaged value from an evaluated compilation such as that of Herron [1999] or Anıcıch [1993] is adopted. The latter compilation has also been used as a guide when there are multiple measured values of an ion-molecule rate coefficient. The transition probabilities for radiative loss processes are listed in Table 4.

The nearly thermoneutral reaction

$$
\mathrm{O}^{+}+\mathrm{H} \rightarrow \mathrm{H}^{+}+\mathrm{O}
$$

deserves special mention. While (R41) is exothermic, and the rate coefficient recommended by Anıcıch [1993] has been adopted, the reverse reaction

$$
\mathrm{H}^{+}+\mathrm{O} \rightarrow \mathrm{O}^{+}+\mathrm{H}
$$

is endothermic for $O$ atoms in the lowest $J=2$ fine structure level, nearly thermoneutral for the $J=1$ level, and exothermic for the highest $J=0$ level. The populations of the fine structure levels is assumed to be determined by the neutral temperature, and we have adopted a rate coefficient for the reverse reaction, $k_{67}$, of

$$
\begin{array}{r}
\frac{k_{f}}{Z} \frac{8}{9}\left[\exp \left(-232.1 / T_{i}\right)+0.6 \exp \left(-228 / T_{n}\right)\right. \\
\left.+0.2 \exp \left(-326 / T_{n}\right)\right]
\end{array}
$$

where $k_{f}$ is the rate coefficient for the forward reaction (R41) and $Z$ is the partition function for the $O$ fine structure levels, which is given by

$$
Z=1+0.6 \exp \left(-228 / T_{n}\right)+0.2 \exp \left(-326 / T_{n}\right) \text {. }
$$

The factor of $8 / 9$ in (1) is the ratio of the electronic degeneracies $(2 L+1)(2 S+1)$ of the products divided by those of the reactants [cf. Banks and Kockarts, 1973].

The rate coefficient for the charge transfer reaction,

$$
\mathrm{O}^{+}\left({ }^{2} D\right)+\mathrm{N}_{2} \rightarrow \mathrm{N}_{2}^{+}+\mathrm{O},
$$

has been recently estimated for temperatures above $\sim 600 \mathrm{~K}$ and found to have a positive temperature dependence by $L i$ et al. [1997b]. The value given in Table 1 is our one-parameter fit to the measured rate coefficients and only applies to ion temperatures below $\sim 4000 \mathrm{~K}$. The fit becomes increasingly inaccurate above $4000 \mathrm{~K}$.

The rate coefficient for the reaction of $\mathrm{N}\left({ }^{4} S\right)$ with $\mathrm{CO}_{2}$,

$$
\mathrm{N}+\mathrm{CO}_{2} \rightarrow \mathrm{NO}+\mathrm{CO},
$$

has been measured several times with widely varying results. Brown and Winkler [1970] and Herron and Huie [1968] reported upper limits of $\sim 1.7 \times 10^{-16} \mathrm{~cm}^{3} \mathrm{~s}^{-1}$. Rawlins and Kaufman [1976], however, placed the upper limit at room temperature considerably lower, at $10^{-19} \mathrm{~cm}^{3} \mathrm{~s}^{-1}$. Lindackers et al. [1990] measured a temperature-dependent rate coefficient for the temperature range $2510-3510 \mathrm{~K}$ of $1.4 \times 10^{-12} \exp (-1114 / T)$. If this rate coefficient is extrapolated to room temperature, a relatively large value of $3.4 \times 10^{-14} \mathrm{~cm}^{3} \mathrm{~s}^{-1}$ is obtained. More recently, however, Fernandez et al. [1998] obtained upper limits to the rate coefficient from 285 to $1142 \mathrm{~K}$; they reported a value at the former temperature of $1.1 \times 10^{-17} \mathrm{~cm}^{3} \mathrm{~s}^{-1}$. For our standard model we adopt a rate coefficient of $1.7 \times 10^{-16} \mathrm{~cm}^{3} \mathrm{~s}^{-1}$ and then test the sensitivity of the model to this rate coefficient by reducing it by a factor of $10^{3}$.

The rate coefficient for (R82),

$$
\mathrm{N}+\mathrm{NO} \rightarrow \mathrm{N}_{2}+\mathrm{O}
$$

in various temperature regimes has been the subject of several studies for a number of years. Lee et al. [1978] measured a temperature-independent rate coefficient for the temperature range $200-400 \mathrm{~K}$ of $3.4 \times$ $10^{-11} \mathrm{~cm}^{3} \mathrm{~s}^{-1}$, which has been recommended by the Jet Propulsion Laboratory (JPL) evaluation [DeMore et al., 1992, 1994]. At high temperatures, other measurements have shown positive temperature dependences [Michael and Lim, 1992; Davidson and Hanson, 1990; Clyne and McDermid, 1975]. Models of the terrestrial thermosphere have also shown better agreement of NO densities if the rate coefficient is larger at temperatures that exceed $300 \mathrm{~K}$ [Siskind and Rusch, 1992]. From models of the Martian lower thermosphere, Fox [1994] suggested that the rate coefficient may have a small positive temperature dependence also at temperatures lower than $300 \mathrm{~K}$. Quasi-classical trajectory calculations of the rate coefficient by Duff and Sharma [1996] did not bear out either the terrestrial or Martian model studies but showed excellent agreement with the JPL recommended rate at room temperature. We have therefore adopted the JPL recommended value of Lee et al. [1978] for this study, but we do not consider this issue to be completely resolved yet.

\section{Production Rates}

Figure 3 shows the ion production rates for $\mathrm{CO}_{2}^{+}, \mathrm{N}_{2}^{+}$, $\mathrm{CO}^{+}, \mathrm{O}^{+}\left({ }^{4} S\right), \mathrm{O}^{+}\left({ }^{2} D\right), \mathrm{O}^{+}\left({ }^{2} P\right), \mathrm{NO}^{+}, \mathrm{C}^{+}, \mathrm{N}^{+}, \mathrm{O}_{2}^{+}$, $\mathrm{H}^{+}$, and $\mathrm{He}^{+}$from photoionization and photoelectron 
Table 1. Ion-Neutral Reaction Rate Coefficients ${ }^{\mathrm{a}}$

\begin{tabular}{|c|c|c|c|}
\hline Reaction No. & Reaction & Rate Coefficient, $\mathrm{cm}^{3} \mathrm{~s}^{-1}$ & References \\
\hline $\begin{array}{l}\text { (R1a) } \\
(\mathrm{R} 1 b) \\
(\mathrm{R} 2)\end{array}$ & $\begin{array}{l}\mathrm{CO}_{2}^{+}+\mathrm{O} \rightarrow \mathrm{CO}+\mathrm{O}_{2}^{+} \\
\mathrm{CO}_{2}^{+}+\mathrm{O} \rightarrow \mathrm{CO}_{2}+\mathrm{O}^{+} \\
\mathrm{CO}_{2}^{+}+\mathrm{O}_{2} \rightarrow \mathrm{CO}_{2}+\mathrm{O}_{2}^{+}\end{array}$ & $\begin{array}{l}1.64 \times 10^{-10} \\
9.60 \times 10^{-11} \\
5.50 \times 10^{-11}\left(300 / T_{i}\right)^{0.82} \\
T_{i} \leq 1500 \mathrm{~K} \\
1.50 \times 10^{-11}\left(T_{i} / 1500\right)^{0.75} \\
T_{i} \geq 1500 \mathrm{~K}\end{array}$ & $\begin{array}{l}\text { Fehsenfeld et al. }[1970] \\
\text { Anicich }[1993] \\
\text { Ferguson et al. }[1992]^{\mathrm{b}}\end{array}$ \\
\hline (R3) & $\mathrm{CO}_{2}^{+}+\mathrm{NO} \rightarrow \mathrm{NO}^{+}+\mathrm{CO}_{2}$ & $1.23 \times 10^{-10}$ & Anicich [1993] \\
\hline (R4) & $\mathrm{CO}_{2}^{+}+\mathrm{N} \rightarrow \mathrm{NO}+\mathrm{CO}^{+}$ & $3.40 \times 10^{-10}$ & Scott et al. [1998] \\
\hline (R5) & $\mathrm{CO}_{2}^{+}+\mathrm{N}\left({ }^{2} D\right) \rightarrow \mathrm{N}^{+}+\mathrm{CO}_{2}$ & $2.00 \times 10^{-10}$ & estimated, see Fox [1982a] \\
\hline (R6) & $\mathrm{CO}_{2}^{+}+\mathrm{H}_{2} \rightarrow \mathrm{HCO}_{2}^{+}+\mathrm{H}$ & $8.70 \times 10^{-10}$ & Scott et al. $[1997]$ \\
\hline (R7a) & $\mathrm{CO}_{2}^{+}+\mathrm{H} \rightarrow \mathrm{HCO}^{+}+\mathrm{O}$ & $4.46 \times 10^{-10}$ & Scott et al. [1997] \\
\hline (R7b) & $\mathrm{CO}_{2}^{+}+\mathrm{H} \rightarrow \mathrm{H}^{+}+\mathrm{CO}_{2}$ & $2.35 \times 10^{-11}$ & \\
\hline (R8) & $\mathrm{CO}^{+}+\mathrm{O} \rightarrow \mathrm{CO}+\mathrm{O}^{+}$ & $1.40 \times 10^{-10}$ & Fehsenfeld and Ferguson [1972] \\
\hline (R9) & $\mathrm{CO}^{+}+\mathrm{NO} \rightarrow \mathrm{CO}+\mathrm{NO}^{+}$ & $4.20 \times 10^{-10}$ & Anicich [1993] \\
\hline (R10) & $\mathrm{CO}^{+}+\mathrm{O}_{2} \rightarrow \mathrm{O}_{2}^{+}+\mathrm{CO}$ & $1.50 \times 10^{-10}\left(300 / T_{i}\right)^{1.1}$ & $\begin{array}{l}\text { Anicich [1993], } \\
\text { Miller et al. }[1984 \mathrm{~b}]^{\mathrm{b}}\end{array}$ \\
\hline (R11) & $\mathrm{CO}^{+}+\mathrm{CO}_{2} \rightarrow \mathrm{CO}_{2}^{+}+\mathrm{CO}$ & $1.10 \times 10^{-9}$ & Anicich [1993] \\
\hline (R12a) & $\mathrm{CO}^{+}+\mathrm{H}_{2} \rightarrow \mathrm{HCO}^{+}+\mathrm{H}$ & $7.50 \times 10^{-10}$ & Scott et al. [1997] \\
\hline (R12b) & $\mathrm{CO}^{+}+\mathrm{H}_{2} \rightarrow \mathrm{HOC}^{+}+\mathrm{H}$ & $7.50 \times 10^{-10}$ & \\
\hline (R13) & $\mathrm{CO}^{+}+\mathrm{H} \rightarrow \mathrm{H}^{+}+\mathrm{CO}$ & $4.00 \times 10^{-10}$ & Scott et al. [1997] \\
\hline (R14) & $\mathrm{CO}^{+}+\mathrm{N} \rightarrow \mathrm{NO}^{+}+\mathrm{C}$ & $8.20 \times 10^{-11}$ & Scott et al. [1998] \\
\hline (R15) & $\mathrm{O}_{2}^{+}+\mathrm{N} \rightarrow \mathrm{NO}^{+}+\mathrm{O}$ & $1.00 \times 10^{-10}$ & Scott et al. [1998] \\
\hline (R16a) & $\mathrm{O}_{2}^{+}+\mathrm{N}\left({ }^{2} D\right) \rightarrow \mathrm{NO}^{+}+\mathrm{O}$ & $1.80 \times 10^{-10}$ & Goldan et al. [1966], \\
\hline (R16b) & $\left.\mathrm{O}_{2}^{+}+\mathrm{N}^{2} D\right) \rightarrow \mathrm{N}^{+}+\mathrm{O}_{2}$ & $8.65 \times 10^{-11}$ & $\begin{array}{l}\text { reverse of (R31b) } \\
\text { O'Keefe et al. }[1986]\end{array}$ \\
\hline (R17) & $\mathrm{O}_{2}^{+}+\mathrm{NO} \rightarrow \mathrm{NO}^{+}+\mathrm{O}_{2}$ & $4.50 \times 10^{-10}$ & Midey and Viggiano [1999] \\
\hline (R18a) & $\mathrm{O}_{2}^{+}+\mathrm{C} \rightarrow \mathrm{CO}^{+}+\mathrm{O}^{-}$ & $5.00 \times 10^{-11}$ & Prasad and Huntress [1980], estimate \\
\hline (R18b) & $\rightarrow \mathrm{C}^{+}+\mathrm{O}_{2}$ & $5.00 \times 10^{-11}$ & \\
\hline (R19) & $\mathrm{O}_{2}^{+}+\mathrm{N}_{2} \rightarrow \mathrm{NO}^{+}+\mathrm{NO}$ & $1.00 \times 10^{-15}$ & Ferguson [1973] \\
\hline (R20) & $\mathrm{N}_{2}^{+}+\mathrm{N} \rightarrow \mathrm{N}^{+}+\mathrm{N}_{2}$ & $1.00 \times 10^{-11}$ & Ferguson [1973] \\
\hline (R21) & $\mathrm{N}_{2}^{+}+\mathrm{CO}_{2} \rightarrow \mathrm{N}_{2}+\mathrm{CO}_{2}^{+}$ & $9.00 \times 10^{-10}\left(300 / T_{i}\right)^{0.23}$ & Dotan et al. [2000] \\
\hline (R22) & $\mathrm{N}_{2}^{+}+\mathrm{CO} \rightarrow \mathrm{N}_{2}^{+}+\mathrm{CO}^{+}$ & $7.60 \times 10^{-11}$ & Frost et al. [1998] \\
\hline (R23) & $\mathrm{N}_{2}^{+}+\mathrm{O}_{2} \rightarrow \mathrm{N}_{2}+\mathrm{O}_{2}^{+}$ & $\begin{array}{l}5.10 \times 10^{-11}\left(300 / T_{i}\right)^{1.16} \\
\text { for } T_{i} \leq 1000 \mathrm{~K} \\
1.26 \times 10^{-11}\left(T_{i} / 1000\right)^{0.67} \\
\text { for } 1000 \leq T_{i} \leq 2000 \mathrm{~K} \\
2.39 \times 10^{-11} \\
\text { for } T_{i} \geq 2000 \mathrm{~K}\end{array}$ & $\begin{array}{l}\text { Scott et al. }[1999] \\
\text { Dotan et al. }[1997]^{\mathrm{b}}\end{array}$ \\
\hline (R24a) & $\mathrm{N}_{2}^{+}+\mathrm{O} \rightarrow \mathrm{NO}^{+}+\mathrm{N}\left({ }^{2} D\right)$ & $\begin{array}{l}1.33 \times 10^{-10}\left(300 / T_{i}\right)^{0.44} \\
\text { for } T_{i} \leq 1500 \mathrm{~K} \\
6.55 \times 10^{-11}\left(1500 / T_{i}\right)^{-0.2} \\
\text { for } T_{i} \geq 1500 \mathrm{~K}\end{array}$ & $\begin{array}{l}\text { Scott et al. }[1999]^{\mathrm{c}} \\
\quad \text { McFarland et al. }[1974]^{\mathrm{b}}\end{array}$ \\
\hline (R24b) & $\mathrm{N}_{2}^{+}+\mathrm{O} \rightarrow \mathrm{O}^{+}+\mathrm{N}_{2}$ & $\begin{array}{l}7.00 \times 10^{-12}\left(300 / T_{i}\right)^{0.23} \\
\text { for } T_{i} \leq 1500 \mathrm{~K} \\
4.83 \times 10^{-12}\left(1500 / T_{i}\right)^{-0.41} \\
\text { for } T_{i} \geq 1500 \mathrm{~K}\end{array}$ & \\
\hline (R25) & $\mathrm{N}_{2}^{+}+\mathrm{NO} \rightarrow \mathrm{N}_{2}+\mathrm{NO}^{+}$ & $3.60 \times 10^{-10}$ & Scott et al. [1999] \\
\hline (R26) & $\mathrm{N}_{2}^{+}+\mathrm{Ar} \rightarrow \mathrm{Ar}^{+}+\mathrm{N}_{2}$ & $1.10 \times 10^{-11} e^{-2089 / T_{2}}$ & reverse of (R71) \\
\hline
\end{tabular}


Table 1. (continued)

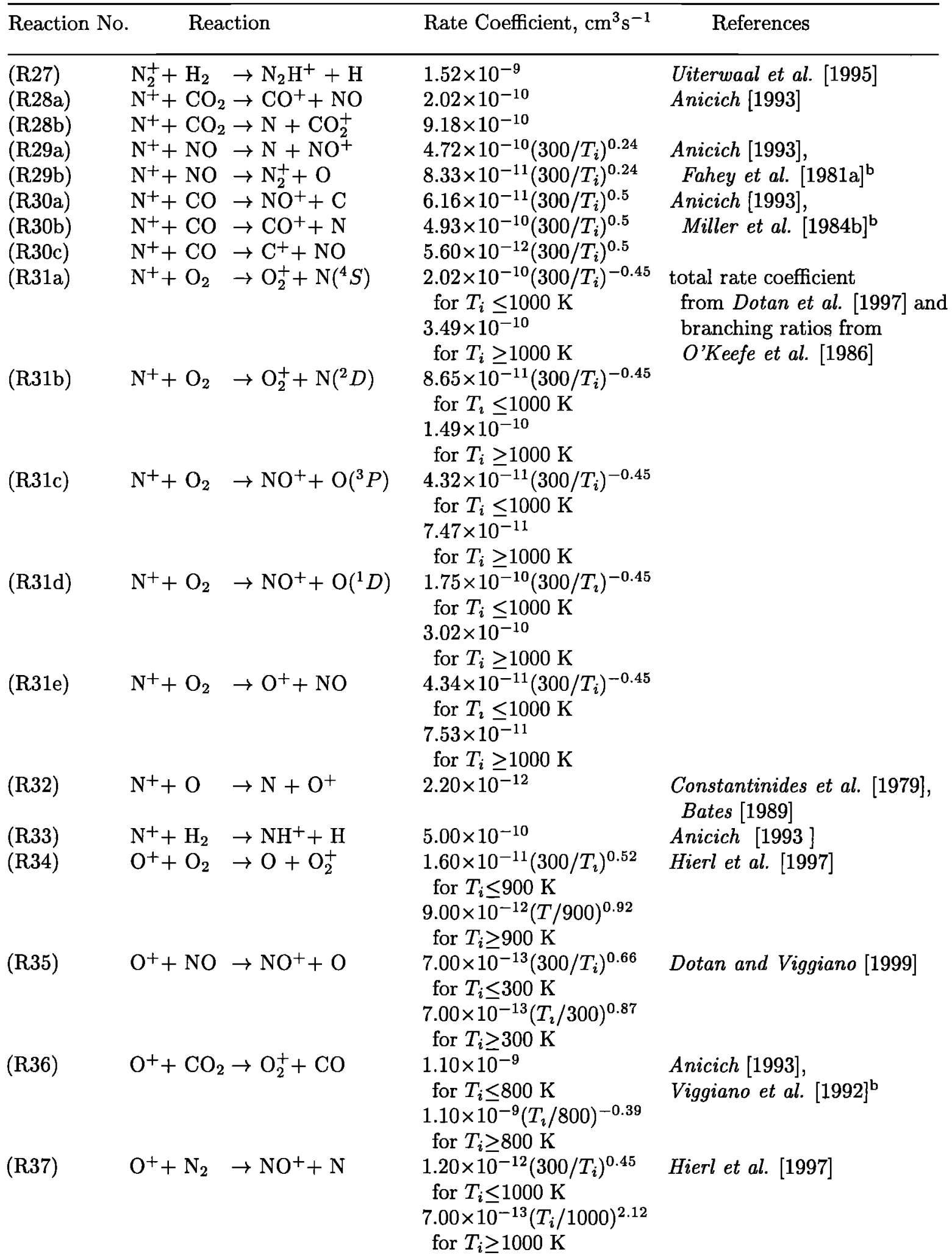


Table 1. (continued)

\begin{tabular}{|c|c|c|c|}
\hline Reaction No & Reaction & Rate Coefficient, $\mathrm{cm}^{3} \mathrm{~s}^{-1}$ & References \\
\hline (R38) & $\left.\mathrm{O}^{+}+\mathrm{N}^{2} D\right) \rightarrow \mathrm{N}^{+}+\mathrm{O}$ & $1.30 \times 10^{-10}$ & $\begin{array}{l}\text { Constantinides et al. }[1979], \\
\text { Bates }[1989]\end{array}$ \\
\hline (R39) & $\mathrm{O}^{+}+\mathrm{C} \rightarrow \mathrm{C}^{+}+\mathrm{O}$ & $1.00 \times 10^{-10}$ & estimate; see text \\
\hline (R40) & $\mathrm{O}^{+}+\mathrm{H}_{2} \rightarrow \mathrm{OH}^{+}+\mathrm{H}$ & $1.35 \times 10^{-9}$ & Li et al. [1997a] \\
\hline (R41) & $\mathrm{O}^{+}+\mathrm{H} \rightarrow \mathrm{H}^{+}+\mathrm{O}$ & $6.40 \times 10^{-10}$ & Anicich [1993] \\
\hline (R42a) & $\mathrm{O}^{+}\left({ }^{2} D\right)+\mathrm{CO}_{2} \rightarrow \mathrm{O}_{2}^{+}+\mathrm{CO}$ & $6.00 \times 10^{-11}$ & Viggiano et al. [1990] \\
\hline (R42b) & $\mathrm{O}^{+}\left({ }^{2} D\right)+\mathrm{CO}_{2} \rightarrow \mathrm{CO}_{2}^{+}+\mathrm{O}$ & $1.00 \times 10^{-9}$ & \\
\hline$(\mathrm{R} 43)^{\prime}$ & $\mathrm{O}^{+}\left({ }^{2} D\right)+\mathrm{CO} \rightarrow \mathrm{CO}^{+}+\mathrm{O}$ & $1.30 \times 10^{-9}$ & Glosik et al. $[1978]$ \\
\hline (R44) & $\mathrm{O}^{+}\left({ }^{2} D\right)+\mathrm{O}_{2} \rightarrow \mathrm{O}_{2}^{+}+\mathrm{O}$ & $7.00 \times 10^{-10}$ & Johnsen and Biondi [1980] \\
\hline (R45) & $\mathrm{O}^{+}\left({ }^{2} D\right)+\mathrm{NO} \rightarrow \mathrm{NO}^{+}+\mathrm{O}$ & $1.20 \times 10^{-9}$ & Glosik et al. [1978] \\
\hline (R46) & $\mathrm{O}^{+}\left({ }^{2} D\right)+\mathrm{O} \rightarrow \mathrm{O}^{+}+\mathrm{O}$ & $1.00 \times 10^{-11}$ & Torr and Torr [1980] \\
\hline (R47) & $\mathrm{O}^{+}\left({ }^{2} D\right)+\mathrm{N}_{2} \rightarrow \mathrm{N}_{2}^{+}+\mathrm{O}$ & $\begin{array}{l}5.70 \times 10^{-10} e^{-400 / T_{i}} \\
\text { for } T_{i} \leq 4000 \mathrm{~K}\end{array}$ & Li et al. $[1997 \mathrm{~b}]^{\mathrm{d}}$ \\
\hline (R48) & $\mathrm{O}^{+}\left({ }^{2} D\right)+\mathrm{N} \rightarrow \mathrm{N}^{+}+\mathrm{O}$ & $1.50 \times 10^{-10}$ & Dalgarno [1979] \\
\hline (R49a) & $\mathrm{O}^{+}\left({ }^{2} D\right)+\mathrm{H}_{2} \rightarrow \mathrm{OH}^{+}+\mathrm{H}$ & $1.50 \times 10^{-9}$ & Li et al. $[1997 \mathrm{a}]$ \\
\hline$(\mathrm{R} 49 \mathrm{~b})$ & $\mathrm{O}^{+}\left({ }^{2} D\right)+\mathrm{H}_{2} \rightarrow \mathrm{H}_{2}^{+}+\mathrm{H}$ & $4.50 \times 10^{-11}$ & \\
\hline (R49c) & $\mathrm{O}^{+}\left({ }^{2} D\right)+\mathrm{H}_{2} \rightarrow \mathrm{H}^{+}+\mathrm{OH}$ & $1.50 \times 10^{-11}$ & \\
\hline (R50) & $\mathrm{O}^{+}\left({ }^{2} D\right)+e \rightarrow \mathrm{O}^{+}\left({ }^{4} S\right)+e$ & $6.03 \times 10^{-8}\left(300 / T_{e}\right)^{0.5}$ & McLaughlin and Bell [1998] \\
\hline (R51a) & $\mathrm{O}^{+}\left({ }^{2} P\right)+\mathrm{CO}_{2} \rightarrow \mathrm{CO}+\mathrm{O}_{2}^{+}$ & $6.00 \times 10^{-11}$ & Viggiano et al. [1990] \\
\hline (R51b) & $\mathrm{O}^{+}\left({ }^{2} P\right)+\mathrm{CO}_{2} \rightarrow \mathrm{CO}_{2}^{+}+\mathrm{O}$ & $1.00 \times 10^{-9}$ & \\
\hline (R52) & $\mathrm{O}^{+}\left({ }^{2} P\right)+\mathrm{CO} \rightarrow \mathrm{CO}^{+}+\mathrm{O}$ & $1.30 \times 10^{-9}$ & Glosik et al. [1978] \\
\hline (R53a) & $\mathrm{O}^{+}\left({ }^{2} P\right)+\mathrm{O}_{2} \rightarrow \mathrm{O}^{+}+\mathrm{O}_{2}$ & $1.30 \times 10^{-10}$ & Glosik et al. [1978] \\
\hline (R53b) & $\mathrm{O}^{+}\left({ }^{2} P\right)+\mathrm{O}_{2} \rightarrow \mathrm{O}_{2}^{+}+\mathrm{O}$ & $1.30 \times 10^{-10}$ & \\
\hline (R54) & $\mathrm{O}^{+}\left({ }^{2} P\right)+\mathrm{O} \rightarrow \mathrm{O}^{+}\left({ }^{2} D\right)+\mathrm{O}$ & $5.20 \times 10^{-10}$ & Rusch et al. $[1977]$ \\
\hline (R55) & $\mathrm{O}^{+}\left({ }^{2} P\right)+\mathrm{N}_{2} \rightarrow \mathrm{O}^{+}+\mathrm{N}_{2}$ & $\begin{array}{l}6.20 \times 10^{-10} e^{-340 / T_{i}} \\
\text { for } T_{i} \leq 4000 \mathrm{~K}\end{array}$ & Li et al. $[1997 \mathrm{~b}]^{\mathrm{d}}$ \\
\hline (R56) & $\mathrm{O}^{+}\left({ }^{2} P\right)+\mathrm{N} \rightarrow \mathrm{O}^{+}+\mathrm{N}\left({ }^{2} D\right)$ & $1.00 \times 10^{-11}$ & $\begin{array}{l}\text { A. Dalgarno (private communication } \\
\text { to Fox }[1982 \mathrm{a}] \text { ) }\end{array}$ \\
\hline (R57) & $\mathrm{O}^{+}\left({ }^{2} P\right)+\mathrm{NO} \rightarrow \mathrm{NO}^{+}+\mathrm{O}$ & $1.20 \times 10^{-9}$ & Glosik et al. $[1978]$ \\
\hline (R58a) & $\mathrm{O}^{+}\left({ }^{2} P\right)+\mathrm{H}_{2} \rightarrow \mathrm{OH}^{+}+\mathrm{H}$ & $8.50 \times 10^{-10}$ & Li et al. $[1997 \mathrm{a}]$ \\
\hline (R58b) & $\mathrm{O}^{+}\left({ }^{2} P\right)+\mathrm{H}_{2} \rightarrow \mathrm{H}_{2}^{+}+\mathrm{H}$ & $3.33 \times 10^{-10}$ & \\
\hline (R58c) & $\mathrm{O}^{+}\left({ }^{2} P\right)+\mathrm{H}_{2} \rightarrow \mathrm{H}^{+}+\mathrm{OH}$ & $6.93 \times 10^{-11}$ & \\
\hline (R58d) & $\mathrm{O}^{+}\left({ }^{2} P\right)+\mathrm{H}_{2} \rightarrow \mathrm{H}^{+}+\mathrm{O}+\mathrm{H}$ & $6.93 \times 10^{-11}$ & \\
\hline (R59a) & $\mathrm{O}^{+}\left({ }^{2} P\right)+e \quad \rightarrow \mathrm{O}^{+}\left({ }^{4} S\right)+e$ & $3.03 \times 10^{-8}\left(300 / T_{e}\right)^{0.5}$ & McLaughlin and Bell [1998] \\
\hline (R59b) & $\mathrm{O}^{+}\left({ }^{2} P\right)+e \rightarrow \mathrm{O}^{+}\left({ }^{2} D\right)+e$ & $1.84 \times 10^{-7}\left(300 / T_{e}\right)^{0.5}$ & \\
\hline$(\mathrm{R} 60)$ & $\mathrm{C}^{+}+\mathrm{CO}_{2} \rightarrow \mathrm{CO}^{+}+\mathrm{CO}^{\prime}$ & $1.10 \times 10^{-9}$ & Fahey et al. $[1981 \mathrm{~b}]$ \\
\hline (R61) & $\mathrm{C}^{+}+\mathrm{NO} \rightarrow \mathrm{NO}^{+}+\mathrm{C}$ & $7.50 \times 10^{-10}\left(300 / T_{i}\right)^{0.2}$ & $\begin{array}{l}\text { Anicich }[1993] \\
\quad \text { Miller et al. }[1984 \mathrm{~b}]^{\mathrm{b}}\end{array}$ \\
\hline (R62a) & $\mathrm{C}^{+}+\mathrm{O}_{2} \rightarrow \mathrm{O}^{+}+\mathrm{CO}$ & $5.22 \times 10^{-10}$ & Anicich [1993], \\
\hline (R62b) & $\mathrm{C}^{+}+\mathrm{O}_{2} \rightarrow \mathrm{CO}^{+}+\mathrm{O}$ & $3.48 \times 10^{-10}$ & Miller et al. $[1984 \mathrm{~b}]^{\mathrm{b}}$ \\
\hline (R63) & $\mathrm{C}^{+}+\mathrm{H}_{2} \rightarrow \mathrm{CH}^{+}+\mathrm{H}$ & $7.40 \times 10^{-10} e^{-4538 / T_{2}}$ & Hierl et al. [1997] \\
\hline (R64) & $\mathrm{H}^{+}+\mathrm{CO}_{2} \rightarrow \mathrm{HCO}^{+}+\mathrm{O}$ & $3.80 \times 10^{-9}$ & Anicich [1993] \\
\hline (R65) & $\mathrm{H}^{+}+\mathrm{O}_{2} \rightarrow \mathrm{O}_{2}^{+}+\mathrm{H}$ & $1.17 \times 10^{-9}$ & Huntress et al. [1974] \\
\hline (R66) & $\mathrm{H}^{+}+\mathrm{NO} \rightarrow \mathrm{H}^{-}+\mathrm{NO}^{+}$ & $1.90 \times 10^{-9}$ & Fehsenfeld and Ferguson [1972] \\
\hline (R67) & $\mathrm{H}^{+}+\mathrm{O} \rightarrow \mathrm{H}+\mathrm{O}^{+}$ & & see text \\
\hline
\end{tabular}


Table 1. (continued)

\begin{tabular}{|c|c|c|c|}
\hline Reaction No. & Reaction & Rate Coefficient, $\mathrm{cm}^{3} \mathrm{~s}^{-1}$ & References \\
\hline$(\mathrm{R} 68)$ & $\mathrm{Ar}^{+}+\mathrm{CO}_{2} \rightarrow \mathrm{Ar}+\mathrm{CO}_{2}^{+}$ & $\begin{array}{l}5.0 \times 10^{-10} \\
\text { for } T_{i} \leq 700 \mathrm{~K} \\
5.0 \times 10^{-10}\left(700 / T_{i}\right) \\
\text { for } T_{i} \geq 700 \mathrm{~K}\end{array}$ & Dotan et al. [1999] \\
\hline$(\mathrm{R} 69)$ & $\mathrm{Ar}^{+}+\mathrm{O}_{2} \rightarrow \mathrm{Ar}+\mathrm{O}_{2}^{+}$ & $\begin{array}{l}4.90 \times 10^{-11}\left(300 / T_{i}\right)^{078} \\
\text { for } T_{i} \leq 900 \mathrm{~K} \\
2.08 \times 10^{-11}\left(T_{i} / 900\right)^{1.65} \\
\quad \text { for } T_{i} \geq 900 \mathrm{~K}\end{array}$ & Midey and Viggiano [1998] \\
\hline$(\mathrm{R} 70)$ & $\mathrm{Ar}^{+}+\mathrm{CO} \rightarrow \mathrm{Ar}+\mathrm{CO}^{+}$ & $\begin{array}{l}3.70 \times 10^{-11}\left(300 / T_{i}\right)^{0.43} \\
\text { for } T_{i} \leq 900 \mathrm{~K} \\
2.30 \times 10^{-11}\left(T_{i} / 900\right) \\
\quad \text { for } T_{i} \geq 900 \mathrm{~K}\end{array}$ & Midey and Viggiano [1998] \\
\hline (R71) & $\mathrm{Ar}^{+}+\mathrm{N}_{2} \rightarrow \mathrm{Ar}+\mathrm{N}_{2}^{+}$ & $1.1 \times 10^{-11}\left(T_{i} / 300\right)^{1.13}$ & $\begin{array}{l}\text { Anicich }[1993] \\
\text { Dotan and Lindinger }[1982]^{\mathrm{b}}\end{array}$ \\
\hline (R72) & $\mathrm{Ar}^{+}+\mathrm{NO} \rightarrow \mathrm{Ar}+\mathrm{NO}^{+}$ & $3.1 \times 10^{-10}$ & Anicich [1993] \\
\hline (R73a) & $\mathrm{Ar}^{+}+\mathrm{H}_{2} \rightarrow \mathrm{H}_{2}^{+}+\mathrm{Ar}$ & $1.78 \times 10^{-11}$ & Anicich [1993] \\
\hline (R73b) & $\mathrm{Ar}^{+}+\mathrm{H}_{2} \rightarrow \mathrm{ArH}^{+}+\mathrm{H}$ & $8.72 \times 10^{-10}$ & \\
\hline (R74) & $\mathrm{He}^{+}+\mathrm{CO} \rightarrow \mathrm{C}^{+}+\mathrm{O}+\mathrm{He}$ & $1.60 \times 10^{-9}$ & Anicich [1993] \\
\hline (R75a) & $\mathrm{He}^{+}+\mathrm{CO}_{2} \rightarrow \mathrm{C}^{+}+\mathrm{O}_{2}+\mathrm{He}$ & $2.00 \times 10^{-11}$ & Anicich [1993] \\
\hline (R75b) & $\mathrm{He}^{+}+\mathrm{CO}_{2} \rightarrow \mathrm{CO}^{+}+\mathrm{O}+\mathrm{He}$ & $7.80 \times 10^{-10}$ & \\
\hline$(\mathrm{R} 75 \mathrm{c})$ & $\mathrm{He}^{+}+\mathrm{CO}_{2} \rightarrow \mathrm{O}^{+}+\mathrm{CO}+\mathrm{He}$ & $1.40 \times 10^{-10}$ & \\
\hline (R75d) & $\mathrm{He}^{+}+\mathrm{CO}_{2} \rightarrow \mathrm{CO}_{2}^{+}+\mathrm{He}$ & $5.00 \times 10^{-11}$ & \\
\hline (R76a) & $\mathrm{He}^{+}+\mathrm{O}_{2} \rightarrow \mathrm{O}^{+}\left({ }^{2} D\right)+\mathrm{O}+\mathrm{He}$ & $2.37 \times 10^{-10}$ & Gerlich [1992], \\
\hline (R76b) & $\mathrm{He}^{+}+\mathrm{O}_{2} \rightarrow \mathrm{O}^{+}+\mathrm{O}+\mathrm{He}$ & $2.39 \times 10^{-11}$ & Bischof and Linder [1986] \\
\hline (R76c) & $\mathrm{He}^{+}+\mathrm{O}_{2} \rightarrow \mathrm{O}_{2}^{+}+\mathrm{O}$ & $9.20 \times 10^{-12}$ & \\
\hline (R76d) & $\mathrm{He}^{+}+\mathrm{O}_{2} \rightarrow \mathrm{O}^{+}\left({ }^{2} P\right)+\mathrm{O}+\mathrm{He}$ & $6.04 \times 10^{-10}$ & \\
\hline (R76e) & $\mathrm{He}^{+}+\mathrm{O}_{2} \rightarrow \mathrm{O}^{+}+\mathrm{O}\left({ }^{1} D\right)+\mathrm{He}$ & $4.60 \times 10^{-11}$ & \\
\hline (R77) & $\mathrm{He}^{+}+\mathrm{O} \rightarrow \mathrm{O}^{+}+\mathrm{He}$ & $1.00 \times 10^{-13}$ & $\begin{array}{l}\text { estimated; } \\
\text { compare Dalgarno and Fox }[199\end{array}$ \\
\hline (R78a) & $\mathrm{He}^{+}+\mathrm{N}_{2} \rightarrow \mathrm{N}^{+}+\mathrm{N}+\mathrm{He}$ & $7.80 \times 10^{-10}$ & Anicich [1993] \\
\hline (R78b) & $\mathrm{He}^{+}+\mathrm{N}_{2} \rightarrow \mathrm{N}_{2}^{+}+\mathrm{He}$ & $5.20 \times 10^{-10}$ & \\
\hline (R79a) & $\mathrm{He}^{+}+\mathrm{NO} \rightarrow \mathrm{N}^{+}+\mathrm{O}+\mathrm{He}$ & $1.35 \times 10^{-9}$ & Anicich [1993] \\
\hline (R79b) & $\mathrm{He}^{+}+\mathrm{NO} \rightarrow \mathrm{O}^{+}+\mathrm{N}+\mathrm{He}$ & $1.00 \times 10^{-10}$ & \\
\hline
\end{tabular}

${ }^{a}$ When the evaluated compilation of Anicich [1993] is cited as a source, the adopted rate coffficient is an average of two or more measurements.

b Room temperature value taken from Anicich [1993]; temperature dependence taken from the second source cited.

c Frederick and Rusch [1977] have suggested that the product $\mathrm{N}$ atoms are in the ${ }^{2} D$ state from models of terrestrial thermosphere. Scott et al. [1999], however, assume that the product $\mathrm{N}$ atoms are in the ground state.

d One-parameter fit to the rate coefficients reported by $L i$ et al. [1997b] for ion temperature less than $4000 \mathrm{~K}$. Above that temperature the fit becomes inaccurate.

impact ionization for the low solar activity model. Figure 4 shows the ion production rates for the high solar activity model.

The major ion production rates peak in the 138-142 km region. $\mathrm{CO}_{2}^{+}$has the largest peak production rates of $1.13 \times 10^{4} \mathrm{~cm}^{-3} \mathrm{~s}^{-1}$ at $141 \mathrm{~km}$ and $2.8 \times 10^{4} \mathrm{~cm}^{-3} \mathrm{~s}^{-1}$ at $138 \mathrm{~km}$, at low and high solar activities, respectively. The solar activity variation is thus a factor of $\sim 2.5$, which closely reflects the variation of the solar ionizing fluxes. The peak production rates of $\mathrm{N}_{2}^{+}$show a similar variation of a factor of slightly more than 2.5 , from $8.3 \times$ $10^{2} \mathrm{~cm}^{-3} \mathrm{~s}^{-1}$ at $142 \mathrm{~km}$ to $2.1 \times 10^{3} \mathrm{~cm}^{-3} \mathrm{~s}^{-1}$ at 139 $\mathrm{km}$. The $\mathrm{O}^{+}\left({ }^{4} S\right)$ peak production rates vary by a factor of $\sim 3$ from $1.97 \times 10^{3}$ to $6.0 \times 10^{3} \mathrm{~cm}^{-3} \mathrm{~s}^{-1}$ from low to high solar activities. Most of the $\mathrm{O}^{+}$produced near the peak is due to dissociative ionization of $\mathrm{CO}_{2}$, and the slightly larger variation than that of the solar EUV fluxes is due to the increase in the mixing ratio 
of $\mathrm{O}$ as solar activity increases. The mixing ratios of $O$ at $140 \mathrm{~km}$ are 0.094 and 0.20 in the low and high solar activity models, respectively. The altitude profiles show that the $\mathrm{O}^{+}\left({ }^{4} S\right)$ production rate near the peak is parallel to that of $\mathrm{CO}_{2}^{+}$, but at higher altitudes the profiles are parallel to the $\mathrm{O}$ density profiles. $\mathrm{O}$ becomes the dominant constituent above $\sim 160 \mathrm{~km}$ at low solar activity and above $\sim 150 \mathrm{~km}$ at high solar activity. A similar effect is seen for $\mathrm{CO}^{+}$, for which the production rates vary by a factor of 3.4 , from $1.79 \times 10^{3}$ to $6.1 \times 10^{3}$

Table 2. Neutral-Neutral Reaction Rate Coefficients

\begin{tabular}{|c|c|c|c|}
\hline Reaction No. & Reaction & Rate Coefficient, $\mathrm{cm}^{3} \mathrm{~s}^{-1}$ & References \\
\hline$(\mathrm{R} 80)$ & $\mathrm{N}+\mathrm{CO}_{2} \rightarrow \mathrm{NO}+\mathrm{CO}$ & & see text \\
\hline (R81) & $\mathrm{N}+\mathrm{O}_{2} \rightarrow \mathrm{NO}+\mathrm{O}$ & $1.50 \times 10^{-14} T_{n} e^{-3270 / T_{n}}$ & Baulch et al. [1994] \\
\hline (R82) & $\mathrm{N}+\mathrm{NO} \rightarrow \mathrm{N}_{2}+\mathrm{O}$ & $3.4 \times 10^{-11}$ & Lee et al. [1978] \\
\hline (R83) & $\mathrm{N}\left({ }^{2} D\right)+\mathrm{CO}_{2} \rightarrow \mathrm{NO}+\mathrm{CO}$ & $3.60 \times 10^{-13}$ & Herron [1999] \\
\hline (R84) & $\mathrm{N}\left({ }^{2} D\right)+\mathrm{CO} \rightarrow \mathrm{N}\left({ }^{4} S\right)+\mathrm{CO}$ & $1.90 \times 10^{-12}$ & Herron [1999] \\
\hline$(\mathrm{R} 85)$ & $\mathrm{N}\left({ }^{2} D\right)+\mathrm{O}_{2} \rightarrow \mathrm{NO}+\mathrm{O}\left({ }^{1} D\right)$ & $9.70 \times 10^{-12} e^{-185 / T_{n}}$ & Herron [1999], Shihira et al. [1994 \\
\hline$(\mathrm{R} 86)$ & $\mathrm{N}\left({ }^{2} D\right)+\mathrm{O} \rightarrow \mathrm{N}\left({ }^{4} S\right)+\mathrm{O}$ & $6.90 \times 10^{-13}$ & Fell et al. [1990] \\
\hline (R87) & $\mathrm{N}\left({ }^{2} D\right)+\mathrm{N}_{2} \rightarrow \mathrm{N}\left({ }^{4} S\right)+\mathrm{N}_{2}$ & $1.70 \times 10^{-14}$ & Herron [1999] \\
\hline (R88) & $\mathrm{N}\left({ }^{2} D\right)+\mathrm{NO} \rightarrow \mathrm{N}_{2}+\mathrm{O}$ & $6.70 \times 10^{-11}$ & Fell et al. [1990] \\
\hline (R89) & $\mathrm{N}\left({ }^{2} D\right)+\mathrm{H}_{2} \rightarrow \mathrm{NH}+\mathrm{H}$ & $4.20 \times 10^{-11} e^{-880 / T_{n}}$ & Herron [1999] \\
\hline (R90) & $\mathrm{N}\left({ }^{2} D\right)+e \rightarrow \mathrm{N}\left({ }^{4} S\right)+e$ & $3.86 \times 10^{-10}\left(T_{e} / 300\right)^{0.81}$ & Berrington and Burke [1981] \\
\hline (R91) & $\mathrm{N}\left({ }^{2} P\right)+\mathrm{CO}_{2} \rightarrow \mathrm{N}\left({ }^{2} D\right)+\mathrm{CO}_{2}$ & $2.00 \times 10^{-15}$ & Herron [1999] \\
\hline (R92) & $\mathrm{N}\left({ }^{2} P\right)+\mathrm{CO} \rightarrow \mathrm{N}\left({ }^{2} D\right)+\mathrm{CO}$ & $6.00 \times 10^{-15}$ & Herron [1999] \\
\hline (R93a) & $\mathrm{N}\left({ }^{2} P\right)+\mathrm{O}_{2} \rightarrow \mathrm{NO}+\mathrm{O}\left({ }^{3} P\right)$ & $1.03 \times 10^{-12} e^{-60 / T_{n}}$ & Shihira et al. [1994], \\
\hline (R93b) & $\mathrm{N}\left({ }^{2} P\right)+\mathrm{O}_{2} \rightarrow \mathrm{NO}+\mathrm{O}\left({ }^{1} D\right)$ & $1.03 \times 10^{-12} e^{-60 / T_{n}}$ & branching ratios from \\
\hline (R93c) & $\mathrm{N}\left({ }^{2} P\right)+\mathrm{O}_{2} \rightarrow \mathrm{NO}+\mathrm{O}\left({ }^{1} S\right)$ & $1.03 \times 10^{-12} e^{-60 / T_{n}}$ & Rawlins et al. [1989] \\
\hline (R94) & $\mathrm{N}\left({ }^{2} P\right)+\mathrm{O} \rightarrow \mathrm{N}\left({ }^{2} D\right)+\mathrm{O}$ & $1.70 \times 10^{-11}$ & Piper $[1993]$ \\
\hline (R95) & $\mathrm{N}\left({ }^{2} P\right)+\mathrm{NO} \rightarrow \mathrm{N}\left({ }^{2} D\right)+\mathrm{NO}$ & $2.90 \times 10^{-11}$ & Herron [1999] \\
\hline (R96) & $\mathrm{N}\left({ }^{2} P\right)+\mathrm{N}_{2} \rightarrow \mathrm{N}\left({ }^{2} D\right)+\mathrm{N}_{2}$ & $5.00 \times 10^{-17}$ & Herron [1999] \\
\hline (R97) & $\mathrm{N}\left({ }^{2} P\right)+\mathrm{N} \rightarrow \mathrm{N}\left({ }^{2} D\right)+\mathrm{N}$ & $6.20 \times 10^{-13}$ & Young and Dunn [1975] \\
\hline (R98) & $\mathrm{N}\left({ }^{2} P\right)+\mathrm{H}_{2} \rightarrow \mathrm{N}\left({ }^{2} D\right)+\mathrm{H}_{2}$ & $2.50 \times 10^{-15}$ & Herron [1999] \\
\hline (R99a) & $\mathrm{N}\left({ }^{2} P\right)+e \rightarrow \mathrm{N}\left({ }^{4} S\right)+e$ & $2.04 \times 10^{-10}\left(T_{e} / 300\right)^{0.85}$ & Berrington and Burke [1981] \\
\hline (R99b) & $\mathrm{N}\left({ }^{2} P\right)+e \rightarrow \mathrm{N}\left({ }^{2} D\right)+e$ & $9.50 \times 10^{-9}$ & \\
\hline (R100) & $\mathrm{C}+\mathrm{CO}_{2} \rightarrow \mathrm{CO}+\mathrm{CO}$ & $7.62 \times 10^{-14}\left(T_{n} / 300\right)^{0.5} e^{-3480 / T_{n}}$ & $\begin{array}{l}\text { McElroy and McConnell [1971], } \\
\text { estimate }\end{array}$ \\
\hline (R101a) & $\mathrm{C}+\mathrm{NO} \rightarrow \mathrm{CN}+\mathrm{O}$ & $7.50 \times 10^{-11}\left(T_{n} / 300\right)^{-0.16}$ & Chastaing et al. [2000] \\
\hline (R101b) & $\mathrm{C}+\mathrm{NO} \rightarrow \mathrm{CO}+\mathrm{N}$ & $7.50 \times 10^{-11}\left(T_{n} / 300\right)^{-0.16}$ & \\
\hline (R102) & $\mathrm{C}+\mathrm{O}_{2} \rightarrow \mathrm{CO}+\mathrm{O}$ & $4.90 \times 10^{-11}\left(T_{n} / 300\right)^{-0.32}$ & Chastaing et al. [2000] \\
\hline (R103) & $\mathrm{O}\left({ }^{1} D\right)+\mathrm{CO}_{2} \rightarrow \mathrm{O}\left({ }^{3} P\right)+\mathrm{CO}_{2}$ & $6.80 \times 10^{-11} e^{117 / T_{n}}$ & Streit et al. [1976] \\
\hline (R104) & $\mathrm{O}\left({ }^{1} D\right)+\mathrm{CO} \rightarrow \mathrm{O}\left({ }^{3} P\right)+\mathrm{CO}$ & $3.60 \times 10^{-11}$ & Schofield [1978] \\
\hline (R105) & $\mathrm{O}\left({ }^{1} D\right)+\mathrm{O}_{2} \rightarrow \mathrm{O}\left({ }^{3} P\right)+\mathrm{O}_{2}$ & $3.20 \times 10^{-11} e^{67 / T_{n}}$ & Atkinson et al. [1997] \\
\hline (R106) & $\mathrm{O}\left({ }^{1} D\right)+\mathrm{O} \rightarrow \mathrm{O}\left({ }^{3} P\right)+\mathrm{O}$ & $6.47 \times 10^{-12}\left(T_{n} / 300\right)^{0.14}$ & Jamieson et al. [1992] \\
\hline (R107) & $\mathrm{O}\left({ }^{1} D\right)+\mathrm{N}_{2} \rightarrow \mathrm{O}\left({ }^{3} P\right)+\mathrm{N}_{2}$ & $1.80 \times 10^{-11} e^{107 / T_{n}}$ & Atkinson et al. [1997] \\
\hline (R108) & $\mathrm{O}\left({ }^{1} D\right)+\mathrm{H}_{2} \rightarrow \mathrm{OH}+\mathrm{H}$ & $1.10 \times 10^{-10}$ & Atkinson et al. [1997] \\
\hline (R109) & $\mathrm{O}\left({ }^{1} D\right)+e \rightarrow \mathrm{O}\left({ }^{3} P\right)+e$ & $2.87 \times 10^{-10}\left(T_{e} / 300\right)^{0.91}$ & Berrington and Burke [1981] \\
\hline (R110a) & $\mathrm{O}\left({ }^{1} S\right)+\mathrm{CO}_{2} \rightarrow \mathrm{O}\left({ }^{1} D\right)+\mathrm{CO}_{2}$ & $2.02 \times 10^{-11} e^{-1327 / T_{n}}$ & Capetanakis et al. [1993] \\
\hline (R110b) & $\mathrm{O}\left({ }^{1} S\right)+\mathrm{CO}_{2} \rightarrow \mathrm{O}\left({ }^{3} P\right)+\mathrm{CO}_{2}$ & $1.19 \times 10^{-11} e^{-1327 / T_{n}}$ & \\
\hline (R111a) & $\mathrm{O}\left({ }^{1} S\right)+\mathrm{O}_{2} \rightarrow \mathrm{O}\left({ }^{1} D\right)+\mathrm{O}_{2}$ & $1.36 \times 10^{-12} e^{-815 / T_{n}}$ & Capetanakis et al. [1993] \\
\hline (R111b) & $\mathrm{O}\left({ }^{1} S\right)+\mathrm{O}_{2} \rightarrow \mathrm{O}\left({ }^{3} P\right)+\mathrm{O}_{2}$ & $3.04 \times 10^{-12} e^{-815 / T_{n}}$ & \\
\hline (R112) & $\mathrm{O}\left({ }^{1} S\right)+\mathrm{O} \rightarrow \mathrm{O}\left({ }^{1} D\right)+\mathrm{O}$ & 0.00 & \\
\hline (R113) & $\mathrm{O}\left({ }^{1} S\right)+\mathrm{N}_{2} \rightarrow \mathrm{O}\left({ }^{1} D\right)+\mathrm{N}_{2}$ & $5.00 \times 10^{-17}$ & Atkinson and Welge [1972] \\
\hline (R114) & $\mathrm{O}\left({ }^{1} S\right)+\mathrm{CO} \rightarrow \mathrm{O}\left({ }^{1} D\right)+\mathrm{CO}$ & $7.40 \times 10^{-14} e^{-961 / T_{n}}$ & Capetanakis et al. [1993] \\
\hline (R115) & $\mathrm{O}\left({ }^{1} S\right)+\mathrm{H}_{2} \rightarrow \mathrm{O}\left({ }^{1} D\right)+\mathrm{H}_{2}$ & $2.86 \times 10^{-16}$ & Capetanakis et al. [1993] \\
\hline (R116a) & $\mathrm{O}\left({ }^{1} S\right)+e \rightarrow \mathrm{O}\left({ }^{1} D\right)+e$ & $8.50 \times 10^{-9}$ & Berrington and Burke [1981] \\
\hline (R116b) & $\mathrm{O}\left({ }^{1} S\right)+e \rightarrow \mathrm{O}\left({ }^{3} P\right)+\mathrm{e}$ & $1.56 \times 10^{-10}\left(T_{e} / 300\right)^{0.94}$ & \\
\hline (R117) & $\mathrm{H}+\mathrm{H}+\mathrm{CO}_{2} \rightarrow \mathrm{H}_{2}+\mathrm{CO}_{2}$ & $1.20 \times 10^{-32}\left(T_{n} / 300\right)^{-1.3}\left(\mathrm{~cm}^{6} \mathrm{~s}\right.$ & Tsang and Hampson [1986] \\
\hline
\end{tabular}


Table 3. Dissociative Recombination Rate Coefficients

\begin{tabular}{|c|c|c|c|}
\hline Reaction No. & Reaction & Rate Coefficient, $\mathrm{cm}^{3} \mathrm{~s}^{-1}$ & References \\
\hline (R118) & $\mathrm{CO}_{2}^{+}+e \rightarrow \mathrm{CO}+\mathrm{O}$ & $3.50 \times 10^{-7}\left(300 / T_{e}\right)^{0.5}$ & Gougousi et al. [1997] \\
\hline (R119a) & $\mathrm{CO}^{+}+e \rightarrow \mathrm{C}+\mathrm{O}$ & $1.80 \times 10^{-7}\left(300 / T_{e}\right)^{0.55}$ & Rosén et al. $[1998]^{\mathrm{a}}$ \\
\hline (R119b) & $\mathrm{CO}^{+}+e \rightarrow \mathrm{C}+\mathrm{O}\left({ }^{1} D\right)$ & $0.25 \times 10^{-7}\left(300 / T_{e}\right)^{0.55}$ & \\
\hline$(\mathrm{R} 119 \mathrm{c})$ & $\mathrm{CO}^{+}+e \rightarrow \mathrm{C}\left({ }^{1} D\right)+\mathrm{O}$ & $0.70 \times 10^{-7}\left(300 / T_{e}\right)^{0.55}$ & \\
\hline \multirow[t]{2}{*}{ (R120a) } & $\mathrm{O}_{2}^{+}+e \rightarrow \mathrm{O}\left({ }^{3} P\right)+\mathrm{O}\left({ }^{3} P\right)$ & $\begin{array}{l}0.39 \times 10^{-7}\left(300 / T_{e}\right)^{0.70} \\
\text { for } T_{e} \leq 1200 \mathrm{~K}\end{array}$ & Alge et al. [1983] \\
\hline & & $\begin{array}{l}1.48 \times 10^{-8}\left(1200 / T_{e}\right)^{0.56} \\
\quad \text { for } T_{e} \geq 1200 \mathrm{~K}\end{array}$ & Mehr and Biondi [1969] \\
\hline (R120b) & $\mathrm{O}_{2}^{+}+e \rightarrow \mathrm{O}\left({ }^{3} P\right)+\mathrm{O}\left({ }^{1} D\right)$ & $\begin{array}{l}0.86 \times 10^{-7}\left(300 / T_{e}\right)^{0.70} \\
\text { for } T_{e} \leq 1200 \mathrm{~K} \\
3.25 \times 10^{-8}\left(1200 / T_{e}\right)^{0.56} \\
\quad \text { for } T_{e} \geq 1200 \mathrm{~K}\end{array}$ & $\begin{array}{l}\text { branching ratios from } \\
\text { Kella et al. }[1997]\end{array}$ \\
\hline (R120c) & $\mathrm{O}_{2}^{+}+e \rightarrow \mathrm{O}\left({ }^{1} D\right)+\mathrm{O}\left({ }^{1} D\right)$ & $\begin{array}{l}6.05 \times 10^{-8}\left(300 / T_{e}\right)^{0.70} \\
\text { for } T_{e} \leq 1200 \mathrm{~K} \\
2.29 \times 10^{-8}\left(1200 / T_{e}\right)^{0.56} \\
\text { for } T_{e} \geq 1200 \mathrm{~K}\end{array}$ & \\
\hline (R120d) & $\mathrm{O}_{2}^{+}+e \rightarrow \mathrm{O}\left({ }^{1} D\right)+\mathrm{O}\left({ }^{1} S\right)$ & $\begin{array}{l}9.75 \times 10^{-9}\left(300 / T_{e}\right)^{0.70} \\
\text { for } T_{e} \leq 1200 \mathrm{~K} \\
3.69 \times 10^{-9}\left(1200 / T_{e}\right)^{0.56} \\
\text { for } T_{e} \geq 1200 \mathrm{~K}\end{array}$ & \\
\hline (R121a) & $\mathrm{N}_{2}^{+}+e \rightarrow \mathrm{N}+\mathrm{N}\left({ }^{2} D\right)$ & $1.01 \times 10^{-7}\left(300 / T_{e}\right)^{0.39}$ & Zipf [1980], \\
\hline (R121b) & $\mathrm{N}_{2}^{+}+e \rightarrow \mathrm{N}\left({ }^{2} D\right)+\mathrm{N}\left({ }^{2} D\right)$ & $1.01 \times 10^{-7}\left(300 / T_{e}\right)^{0.39}$ & branching ratios from \\
\hline (R121c) & $\mathrm{N}_{2}^{+}+e \rightarrow \mathrm{N}+\mathrm{N}\left({ }^{2} P\right)$ & $1.76 \times 10^{-8}\left(300 / T_{e}\right)^{0.39}$ & Kella et al. [1996] \\
\hline (R122a) & $\mathrm{NO}^{+}+e \rightarrow \mathrm{N}\left({ }^{2} D\right)+\mathrm{O}$ & $3.40 \times 10^{-7}\left(300 / T_{e}\right)^{0.5}$ & Vejby-Christensen et al. [1998] \\
\hline (R122b) & $\mathrm{NO}^{+}+e \rightarrow \mathrm{N}+\mathrm{O}$ & $0.60 \times 10^{-7}\left(300 / T_{e}\right)^{0.5}$ & \\
\hline
\end{tabular}

${ }^{a}$ Branching ratios interpolated at $2500 \mathrm{~K}$ from their values measured at the collision energy of $0.0,0.4,1.0$, and $1.5 \mathrm{eV}$.

$\mathrm{cm}^{-3} \mathrm{~s}^{-1}$ from low to high solar activity. The slight enhancement in the production rates over the variation of the solar EUV fluxes is due to the increase in the mixing ratio of $\mathrm{CO}$, which is $6.6 \%$ in the solar minimum model and $11.6 \%$ in the solar maximum model at 140 km.

The $\mathrm{NO}^{+}$production rate profile is a superposition of two peaks. The upper peak in the 138-141 km range, which is evident as a shoulder in the total production rate profile (Figures $3 \mathrm{~b}$ and $4 \mathrm{~b}$ ), is due to ionization by solar EUV photons and photoelectrons. A larger lower peak appears near $117-119 \mathrm{~km}$ and results from photoionization of NO by Lyman alpha. The variation of the production rate near the upper peak is a factor of 2.5 , and near the lower peak it is a factor of 3.8. This low-altitude enhancement is larger than the variation of the solar Lyman alpha line, which is a factor of $\sim 2.9$ in the Hinteregger spectra. This is mostly because the

Table 4. Transition Probabilities A

\begin{tabular}{llll}
\hline Wavelength, $\AA$ & \multicolumn{1}{c}{ Transition } & $\mathrm{A}, \mathrm{s}^{-1}$ & \multicolumn{1}{c}{ Reference } \\
& & & \\
\hline 3728,3726 & $\mathrm{O}^{+}\left({ }^{2} D\right) \rightarrow \mathrm{O}^{+}\left({ }^{4} S\right)+h \nu$ & $4.85 \times 10^{-5}$ & Seaton and Osterbrock $[1957]$ \\
7319,7329 & $\mathrm{O}^{+}\left({ }^{2} P\right) \rightarrow \mathrm{O}^{+}\left({ }^{2} D\right)+h \nu$ & $1.71 \times 10^{-1}$ & Seaton and Osterbrock [1957] \\
2470 & $\mathrm{O}^{+}\left({ }^{2} P\right) \rightarrow \mathrm{O}^{+}\left({ }^{4} S\right)+h \nu$ & $4.8 \times 10^{-2}$ & Seaton and Osterbrock [1957] \\
5200 & $\mathrm{~N}\left({ }^{2} D\right) \rightarrow \mathrm{N}\left({ }^{4} S\right)+h \nu$ & $1.07 \times 10^{-5}$ & Wiese et al. $[1966]$ \\
10400 & $\mathrm{~N}\left({ }^{2} P\right) \rightarrow \mathrm{N}\left({ }^{2} D\right)+h \nu$ & $7.9 \times 10^{-2}$ & Wiese et al. $[1966]$ \\
3466 & $\mathrm{~N}\left({ }^{2} P\right) \rightarrow \mathrm{N}\left({ }^{4} S\right)+h \nu$ & $5.0 \times 10^{-3}$ & Wiese et al. $[1966]$ \\
6300,6364 & $\mathrm{O}\left({ }^{1} D\right) \rightarrow \mathrm{O}\left({ }^{3} P\right)+h \nu$ & $9.3 \times 10^{-3}$ & Froese-Fischer and Saha $[1983]$ \\
5577 & $\mathrm{O}\left({ }^{1} S\right) \rightarrow \mathrm{O}\left({ }^{1} D\right)+h \nu$ & 1.06 & Kernahan and Pang $[1975]$ \\
2972 & $\mathrm{O}\left({ }^{1} S\right) \rightarrow \mathrm{O}\left({ }^{3} P\right)+h \nu$ & $4.5 \times 10^{-2}$ & Kernahan and Pang $[1975]$ \\
\hline
\end{tabular}



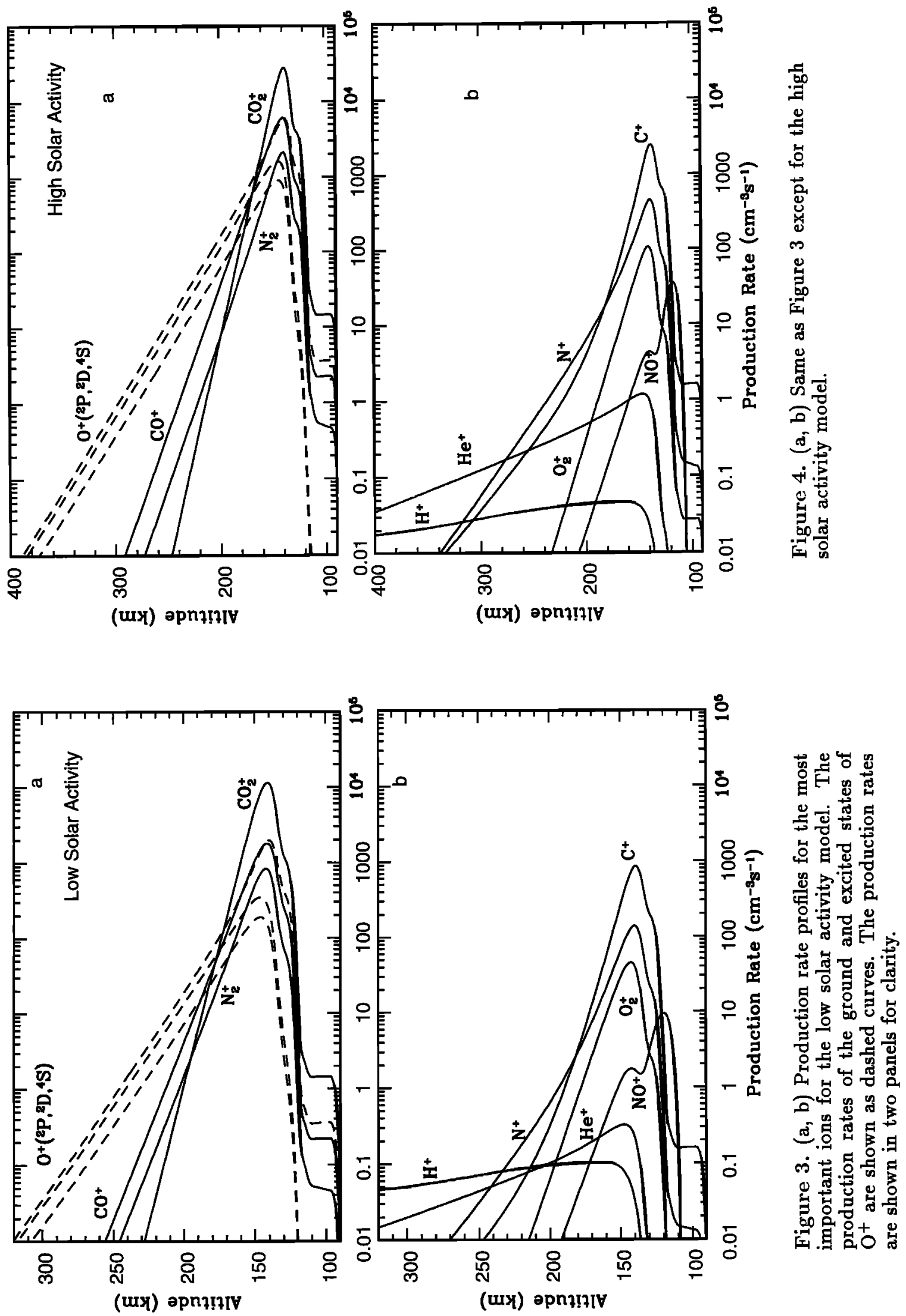
NO density at the high solar activity peak at $117 \mathrm{~km}$ is larger than that at the low solar activity peak at $119 \mathrm{~km}$, even though, as we shall demonstrate in section 5.2, the NO profile itself shows little solar activity variation. It should, however, be noted that chemical reactions rather than direct ionization of $\mathrm{NO}$ are the major sources of $\mathrm{NO}^{+}$over most of the altitude range considered here.

Because the $\mathrm{H}$ densities are anticorrelated with the solar flux and the variation of those densities is larger than that of the solar EUV flux, the $\mathrm{H}^{+}$peak production rate decreases from $1.04 \times 10^{-1} \mathrm{~cm}^{-3} \mathrm{~s}^{-1}$ near 165 $\mathrm{km}$ at low solar activity to $4.6 \times 10^{-2}$ at $169 \mathrm{~km}$ at high solar activity. The altitude of peak production of $\mathrm{H}^{+}$is higher than that of the heavier ions because it is produced mainly by ionization of $\mathrm{H}$, which has a much smaller mass than the average mass of the atmosphere. Using the same approach as Chapman theory, it can be shown easily that for an isothermal atmosphere with one major and one minor species $j$, the optical depth $\tau$ at which the maximum ionization rate of the minor species occurs is given by

$$
\tau=H / H_{j}
$$

where $H$ is the scale height of the major species that controls the photoabsorption and $H_{j}$ is the scale height of the minor species. Thus, for a species that is much lighter than those that control the photoabsorption, which are $\mathrm{CO}_{2}$ and $\mathrm{O}$ in the lower thermosphere of Venus, the maximum ionization rate occurs at a much smaller optical depth and is therefore higher in the atmosphere than that of the major species, for which the maximum ionization rate is near $\tau=1$.

The $\mathrm{He}^{+}$production rate peak varies by a larger factor of 3.7 , from $0.32 \mathrm{~cm}^{-3} \mathrm{~s}^{-1}$ near $147 \mathrm{~km}$ at low solar activity to $1.2 \mathrm{~cm}^{-3} \mathrm{~s}^{-1}$ at $146 \mathrm{~km}$ at high solar activity. The solar activity variation in the peak $\mathrm{He}^{+}$production rate is enhanced because solar flux variations are larger at wavelengths shorterward of the He ionization threshold at $504.8 \AA$ than at longer wavelengths.

$\mathrm{C}^{+}$is produced at low altitudes by photodissociative ionization and electron impact dissociative ionization of $\mathrm{CO}_{2}$ and $\mathrm{CO}$, and at higher altitudes, $\mathrm{C}^{+}$is produced by ionization of $\mathrm{C}$. The production rates at the peak are $860 \mathrm{~cm}^{-3} \mathrm{~s}^{-1}$ at $139 \mathrm{~km}$ and $2480 \mathrm{~cm}^{-3} \mathrm{~s}^{-1}$ at $137 \mathrm{~km}$, for the low and high solar activity models, respectively. This variation of a factor of 2.9 is slightly enhanced over that of the solar EUV fluxes mostly because the mixing ratio of $\mathrm{CO}$ and therefore the production rate by dissociative ionization are enhanced at high solar activity.

The direct production rate profiles of $\mathrm{O}^{+}\left({ }^{2} D\right)$ and $\mathrm{O}^{+}\left({ }^{2} P\right)$ also exhibit larger variations than that of $\mathrm{O}^{+}\left({ }^{4} S\right)$, since in this model they are assumed to be produced only by photoionization and electron impact ionization of $\mathrm{O}$. For $\mathrm{O}^{+}\left({ }^{2} D\right)$ the peak production rate is $350 \mathrm{~cm}^{-3} \mathrm{~s}^{-1}$ at $146 \mathrm{~km}$ and $1620 \mathrm{~cm}^{-3} \mathrm{~s}^{-1}$ at $144 \mathrm{~km}$, for the low and high solar activity models, respectively. The peak in the $\mathrm{O}^{+}\left({ }^{2} P\right)$ production rate increases from $190 \mathrm{~cm}^{-3} \mathrm{~s}^{-1}$ at $146 \mathrm{~km}$ to $900 \mathrm{~cm}^{-3} \mathrm{~s}^{-1}$ at $144 \mathrm{~km}$. These increases by factors of 4.7-4.8 from low to high solar activities are enhanced over that of the EUV solar fluxes mostly because the $\mathrm{O}$ mixing ratio is larger in the high solar activity model. The altitudes of peak production of the metastable $\mathrm{O}^{+}$ions are slightly higher than that of $\mathrm{O}^{+}\left({ }^{4} S\right)$ because $\mathrm{O}$ is lighter than $\mathrm{CO}_{2}$, from which most of the $\mathrm{O}^{-r}\left({ }^{4} S\right)$ is produced near the peak. The effect is not large, because the optical depths due to $\mathrm{O}$ and to $\mathrm{CO}_{2}$ are comparable in the $140-150 \mathrm{~km}$ range. At the altitude of peak $\mathrm{O}^{+}$production near $140 \mathrm{~km}$ the slant column densities of $\mathrm{O}$ and $\mathrm{CO}_{2}$ are approximately equal in the solar maximum model. For the solar minimum model, the slant column density of $\mathrm{O}$ exceeds that of $\mathrm{CO}_{2}$ above $\sim 150 \mathrm{~km}$.

\section{Results and Discussion}

\subsection{Ion Density Profiles}

We have computed the density profiles of 13 ions, including $\mathrm{CO}_{2}^{+}, \mathrm{Ar}^{+}, \mathrm{N}_{2}^{+}, \mathrm{O}^{+}\left({ }^{4} S\right), \mathrm{O}^{+}\left({ }^{2} D\right), \mathrm{O}^{+}\left({ }^{2} P\right)$, $\mathrm{CO}^{+}, \mathrm{C}^{+}, \mathrm{N}^{+}, \mathrm{O}_{2}^{+}, \mathrm{NO}^{+}, \mathrm{H}^{+}$, and $\mathrm{He}^{+}$. The resulting density profiles are shown in Figures $5 \mathrm{a}$ and $5 \mathrm{~b}$ for low and high solar activities, respectively. The peak ion and electron densities and altitudes at high and low solar activities are summarized in Table 5 . The major ion over the range of $\sim 120-190 \mathrm{~km}$ is $\mathrm{O}_{2}^{+}$, and its density at the peak increases by a factor of $\sim 1.6$, from $2.7 \times 10^{5}$ to $4.3 \times 10^{5} \mathrm{~cm}^{-3}$ from low to high solar activities. This is less than that of the solar flux because of the nature of the loss process for $\mathrm{O}_{2}^{+}$. The major $\mathrm{O}_{2}^{+}$sink near and above the peak is dissociative recombination, and the $\mathrm{O}_{2}^{+}$density does not differ substantially from the total electron density. The density near the peak is thus given by

$$
\left[\mathrm{O}_{2}^{+}\right]=\frac{P}{\alpha[e]} \approx\left(\frac{P}{\alpha}\right)^{1 / 2}
$$

where $P$ is the total production rate (which is approximately equal to the direct ionization rate of $\left.\mathrm{CO}_{2}\right),[e]$ is the electron density, and $\alpha$ is the dissociative recombination coefficient. Thus, near the electron density peak the $\mathrm{O}_{2}^{+}$density is approximately proportional to the square root of the production rate [cf. Cravens et al., 1981].

Below $\sim 120 \mathrm{~km}$ the major ion is $\mathrm{NO}^{+}$. Because the ionization potential of $\mathrm{NO}(9.26 \mathrm{eV})$ is the lowest of the ions in the Venus thermosphere considered here, dissociative recombination is the only loss process. $\mathrm{NO}^{+}$ is produced in many ion-molecule reactions, and direct production by photoionization and electron impact ionization is less important than the chemical sources. The $\mathrm{NO}^{+}$density profile is complex and exhibits two main peaks. The reaction

(R24a)

$$
\mathrm{N}_{2}^{+}+\mathrm{O} \rightarrow \mathrm{NO}^{+}+\mathrm{N}
$$



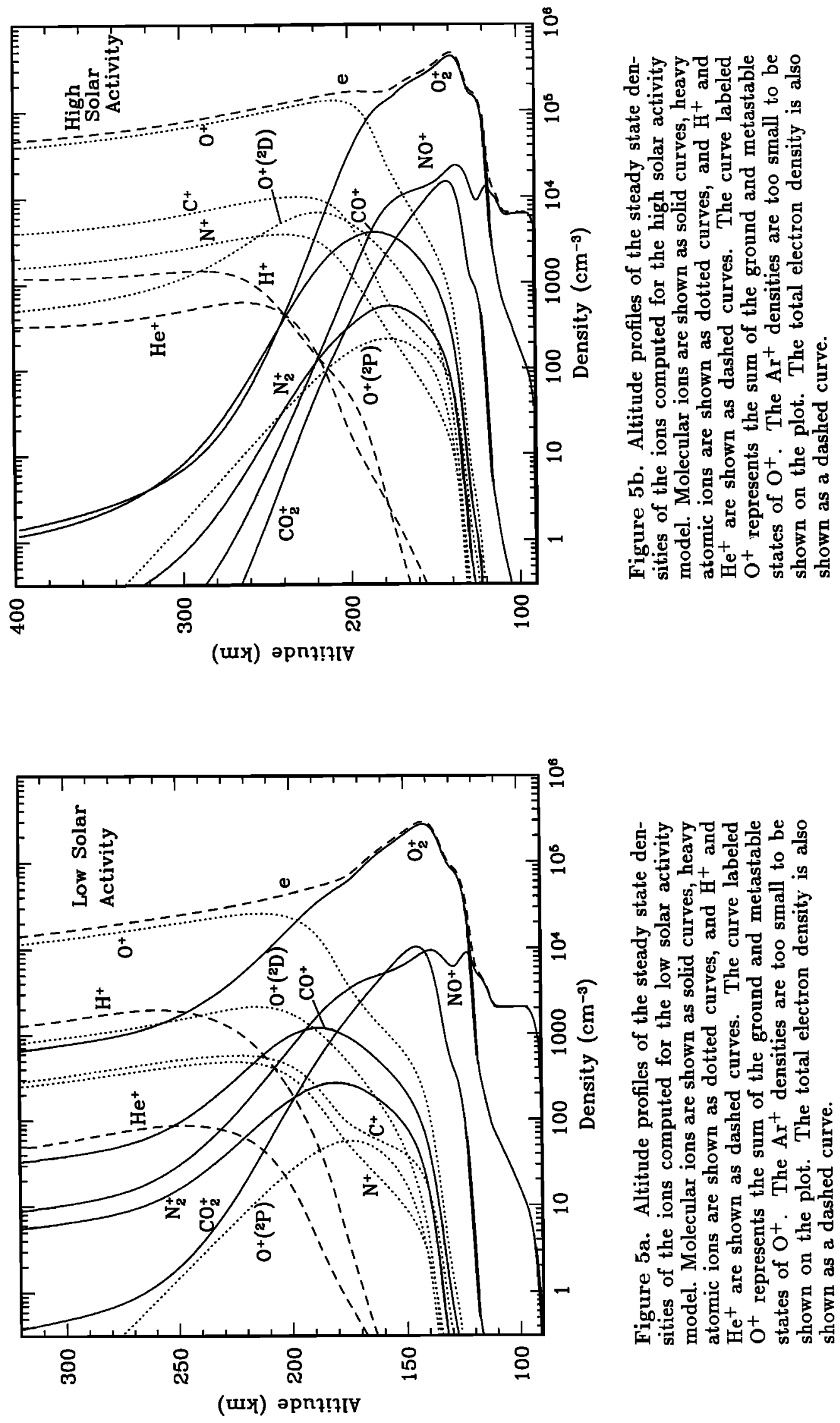
Table 5. Peak Densities at Low and High Solar Activities

\begin{tabular}{|c|c|c|c|c|c|}
\hline \multirow[b]{2}{*}{ Species } & \multicolumn{2}{|c|}{ Peak Altitude, km } & \multicolumn{2}{|c|}{ Peak Density, $\mathrm{cm}^{-3}$} & \multirow[b]{2}{*}{ Factor $^{\mathrm{a}}$} \\
\hline & Low & High & Low & High & \\
\hline $\mathrm{H}^{+}$ & 259 & 289 & $2.0(3)^{b}$ & $1.42(3)$ & 0.71 \\
\hline $\mathrm{CO}_{2}^{+}$ & 145 & 142 & $1.05(4)$ & $1.53(4)$ & 1.5 \\
\hline $\mathrm{NO}^{+} \mathrm{c}$ & 122 & 119 & $8.9(3)$ & $1.32(4)$ & 1.5 \\
\hline Electron & 141 & 139 & $2.9(5)$ & $4.7(5)$ & 1.6 \\
\hline $\mathrm{O}_{2}^{+}$ & 141 & 139 & $2.7(5)$ & $4.3(5)$ & 1.6 \\
\hline $\mathrm{N}_{2}^{+}$ & 179 & 177 & $2.7(2)$ & $5.4(2)$ & 2.0 \\
\hline $\mathrm{NO}^{+} \mathrm{d}$ & 138 & 136 & $9.6(3)$ & $2.4(4)$ & 2.5 \\
\hline $\mathrm{Ar}^{+}$ & 182 & 184 & $1.02(-1)$ & $3.1(-1)$ & 3.0 \\
\hline $\mathrm{O}^{+}\left({ }^{2} D\right)$ & 215 & 219 & $2.1(3)$ & $6.8(3)$ & 3.2 \\
\hline $\mathrm{CO}^{+}$ & 188 & 185 & $1.2(3)$ & $4.0(3)$ & 3.3 \\
\hline $\mathrm{O}^{+}\left({ }^{2} P\right)$ & 174 & 178 & $5.7(1)$ & $2.3(2)$ & 4.0 \\
\hline $\mathrm{O}^{+}$total & 213 & 209 & $2.6(4)$ & $1.33(5)$ & 5.1 \\
\hline $\mathrm{O}^{+}\left({ }^{4} S\right)$ & 213 & 209 & $2.4(4)$ & $1.27(5)$ & 5.4 \\
\hline $\mathrm{He}^{+}$ & 248 & 263 & 8.8(1) & $6.1(2)$ & 6.9 \\
\hline $\mathrm{N}^{+}$ & 226 & 240 & $4.8(2)$ & $3.8(3)$ & 7.9 \\
\hline $\mathrm{C}^{+}$ & 223 & 231 & $5.8(2)$ & $1.03(4)$ & 18. \\
\hline
\end{tabular}

\footnotetext{
${ }^{a}$ Factor by which peak densities increase from low to high solar activities.

${ }^{b}$ Read as $2.0 \times 10^{3}$.

${ }^{c}$ Lower peak.

dUpper peak.
}

dominates the production of $\mathrm{NO}^{+}$at and above the electron density peak near $140 \mathrm{~km}$ over the entire solar cycle. There is also some contribution from

$$
\mathrm{O}_{2}^{+}+\mathrm{N} \rightarrow \mathrm{NO}^{+}+\mathrm{O}
$$

in the same region. Since the major loss process is dissociative recombination, the specific loss rate is proportional to the electron density and thus increases with solar cycle. Because, however, the densities of the reactants in the production reactions also increase significantly from low to high solar activity, the resulting variation of the peak density of $\mathrm{NO}^{+}$near $136 \mathrm{~km}$ is substantial, a factor of $\sim 2.5$.

There is a lower peak in the $\mathrm{NO}^{+}$density profile near $120 \mathrm{~km}$ that varies by a factor of 1.5 over the solar cycle. Although there is also a maximum in the direct production rate near this altitude that is due to photoionization of NO by solar Lyman alpha photons, the density peak is not due to this process. $\mathrm{NO}^{+}$is produced largely by charge transfer from $\mathrm{O}_{2}^{+}$to NO (reaction (R17)) in the lower ionosphere. The lower maximum results from the competition between decreasing $\mathrm{O}_{2}^{+}$densities and increasing $\mathrm{NO}$ densities as altitude decreases. There is, however, some fine-scale structure visible in the lower peaks near $118 \mathrm{~km}$, which is produced by Lyman alpha photoionization of NO.

The variation of the density of $\mathrm{NO}^{+}$is a factor of 3.1 below $\sim 110 \mathrm{~km}$ in the model, where the major source is ionization of the neutral constituents by $X$ rays in the 0.25-18 $\AA$ region and their high-energy photoelectrons, and subsequent charge transfer and other ion-molecule reactions that terminate in $\mathrm{NO}^{+}$. Since $\mathrm{NO}^{+}$in this region also is destroyed by dissociative recombination, and it is the principal ion, the variation is approximately the square root of the assumed factor of 10 variation in the solar X ray fluxes below $18 \AA$.

The peak densities of $\mathrm{N}_{2}^{+}$and $\mathrm{CO}^{+}$occur at higher altitudes than the other molecular ions because the major loss processes for these ions over most of the ionosphere are reactions with neutral species rather than dissociative recombination. $\mathrm{N}_{2}^{+}$and $\mathrm{CO}^{+}$have relatively high ionization potentials of 15.58 and $14.01 \mathrm{eV}$, so that many charge transfer and other ion-molecule reactions with the major neutral species in the Venus thermosphere are exothermic. The variation of the peak $\mathrm{N}_{2}^{+}$density is $270 \mathrm{~cm}^{-3}$ at $179 \mathrm{~km}$ to $540 \mathrm{~cm}^{-3}$ near $177 \mathrm{~km}$ from low to high solar activity. This factor of 2 change is slightly less than the variation of the production rates, because the loss mechanisms also increase with solar activity. The major loss processes are reactions with $\mathrm{CO}_{2}$ at low altitudes and with $\mathrm{O}$ at high altitudes. Since the $O$ mixing ratios are larger at high solar activity, the loss rates are also enhanced at high solar activity. The $\mathrm{CO}^{+}$profiles exhibit peaks of $1.20 \times 10^{3}$ at $188 \mathrm{~km}$ and $4.0 \times 10^{3} \mathrm{~cm}^{-3}$ at $185 \mathrm{~km}$ at low and high solar activities, respectively. This variation of a factor of 3.3 is greater than that of the solar flux, because the CO mixing ratios are larger in the high solar activity model than in the low solar activity model. Reaction with $\mathrm{O}$ is the most important sink for $\mathrm{CO}^{+}$at these altitudes. Dissociative recombination dominates the loss of $\mathrm{N}_{2}^{+}$and $\mathrm{CO}^{+}$only above altitudes of 210 and $220 \mathrm{~km}$, respectively, at high solar activity, and at slightly higher 
altitudes at low solar activity. It is noteworthy that the peak total mass-28 ion density in the model is about half those measured by the PV OIMS measurements at high solar activity, which are of the order of $10^{4} \mathrm{~cm}^{-3}$ or more [e.g., Taylor et al., 1980] and less than in previous models [e.g., Fox, 1982a; Paxton, 1988]. The model of Fox [1982a] exhibited peak total mass-28 ion densities of $\sim 7 \times 10^{3} \mathrm{~cm}^{-3}$. The difference is due to our adoption of recently measured values for the rate coefficients for reactions of $\mathrm{O}^{+}\left({ }^{2} D\right)$ and $\mathrm{O}^{+}\left({ }^{2} P\right)$ with $\mathrm{CO}_{2}$ (reactions (R42) and (R51)) by Viggiano et al. [1990] and for reactions of $\mathrm{O}^{+}\left({ }^{2} D\right)$ and $\mathrm{O}^{+}\left({ }^{2} P\right)$ with $\mathrm{N}_{2}$ (reactions (R47) and (R55)) by Li et al. [1997b]. Viggiano et al. did not consider the channel of (R42) that produces $\mathrm{CO}^{+}$, and their mass resolution was coarse, but they concluded that the major channel by which the reaction proceeds is charge transfer. $\mathrm{Li}$ et al. argued that the nearly gas kinetic rate coefficient for (R47) measured by Johnsen and Brondr [1980] was inaccurate because of the difficulty of estimating the fraction of $\mathrm{O}^{+}\left({ }^{2} D\right)$ in their experiment. Li et al., however, consider their own cross sections and derived rate coefficients to be "estimates" rather than "measurements." Nonetheless, the rate coefficients that we are using here are the best available, and it appears that there is again a significant discrepancy in the mass- 28 ion densities measured by the PV OIMS and the model densities, which deserves a closer look.

The solar activity variations of the peak densities of $\mathrm{N}^{+}$and total $\mathrm{O}^{+}\left(\mathrm{O}^{+}\left({ }^{4} S\right)+\mathrm{O}^{+}\left({ }^{2} D\right)+\mathrm{O}^{+}\left({ }^{2} P\right)\right)$, factors of 7.9 and 5.1, respectively, are enhanced over those of the solar EUV fluxes because the mixing ratios and thus the densities of $\mathrm{N}$ and $\mathrm{O}$ increase also with solar activity. The $\mathrm{N}^{+}$peak densities in the low and high solar activity models of 480 and $3.8 \times 10^{3} \mathrm{~cm}^{-3}$, respectively, occur at 226 and $240 \mathrm{~km}$, respectively. The peak densities of $\mathrm{O}^{+}\left({ }^{4} S\right)$ are $2.4 \times 10^{4} \mathrm{~cm}^{-3}$ at $213 \mathrm{~km}$ and $1.27 \times 10^{5} \mathrm{~cm}^{-3}$ at $209 \mathrm{~km}$, for the low and high solar activity models, respectively. As Figures $5 \mathrm{a}$ and $5 \mathrm{~b}$ show, the high-altitude density variations of the electronically excited ions $\mathrm{O}^{+}\left({ }^{2} D\right)$ and $\mathrm{O}^{+}\left({ }^{2} P\right)$ are smaller than that of $\mathrm{O}^{+}\left({ }^{4} S\right)$. The solar activity variations of the excited states at low altitudes are larger than that of the ground state because they are assumed to be produced by direct ionization only and not by dissociative ionization of $\mathrm{CO}_{2}$ or $\mathrm{CO}$. The excited states of $\mathrm{O}^{+}$, like other ions whose parent neutrals have high ionization potentials, react at low altitudes with most of the neutral species in the Venus thermosphere, including species with high ionization potentials, such as $\mathrm{N}_{2}$ and $\mathrm{H}_{2}$.

The atomic ion peaks form at high altitudes because the only chemical loss processes are reactions with neutrals, whose densities decrease with altitude. As is well known, the atomic ion densities would increase with altitude indefinitely, if diffusion did not eventually become more important as a loss mechanism than chemical reactions. Thus $F_{2}$ peaks in the atomic ion density profiles form where the lifetime for loss by diffusion is equal to that for chemical loss. These peaks in the atomic ions barely appear in the total electron density profile in the low solar activity model, but a prominent shoulder appears at the $\mathrm{O}^{+}$peak in the high solar activity model. This will be discussed further below.

The peak $\mathrm{C}^{+}$density varies from $575 \mathrm{~cm}^{-3} \mathrm{~s}^{-1}$ at $223 \mathrm{~km}$ in the low solar activity model to $1.03 \times 10^{4}$ $\mathrm{cm}^{-3} \mathrm{~s}^{-1}$ at $231 \mathrm{~km}$ in the high solar activity model. This large variation of a factor of 18 is due to the synergistic effect of several factors. The major source of $\mathrm{C}^{+}$near the peak in the low solar activity model is photoionization of $\mathrm{C}$. The $\mathrm{C}$ density varies by factor of $\sim 9$ near the $\mathrm{C}^{+}$peak from low to high solar activity. (The solar activity variation of atomic carbon will be discussed in section 5.2.) At high solar activity a second source becomes important, charge transfer of $\mathrm{O}^{+}$ to $\mathrm{C}$ :

$$
\mathrm{O}^{+}+\mathrm{C} \rightarrow \mathrm{C}^{+}+\mathrm{O}
$$

Although the rate coefficient for this reaction has not been measured, our assumed value of $1 \times 10^{-10} \mathrm{~cm}^{3} \mathrm{~s}^{-1}$ is approximately that required to reproduce the maxima in the $\mathrm{C}^{+}$profiles, which are of the order of $10^{4}$ $\mathrm{cm}^{-3}$, as measured by the PV OIMS at high solar activity [e.g., Taylor et al., 1980; Fox and Fllore, 1997]. As we have shown above, the $\mathrm{O}^{+}$densities also vary substantially with solar activity; near the $\mathrm{C}^{+}$peaks, the $\mathrm{O}^{+}$densities increase by a factor of $\sim 5$ from low to high solar activity. From the lower ionosphere to above the peak, the major chemical loss process for $\mathrm{C}^{+}$is its reaction with $\mathrm{CO}_{2}$ :

$$
\mathrm{C}^{+}+\mathrm{CO}_{2} \rightarrow \mathrm{CO}^{+}+\mathrm{CO} \text {. }
$$

The $\mathrm{CO}_{2}$ densities, however, increase by only a factor of 2-4 from low to high solar activities in the 200-225 $\mathrm{km}$ altitude range in the VTS3 models, and thus the loss rates do not increase enough to compensate for the large increase in the production rates.

At high altitudes the solar cycle variation of the molecular ion densities is opposite to that of the atomic ions; that is, they are anticorrelated with the solar flux. At $300 \mathrm{~km}$ the $\mathrm{O}_{2}^{+}, \mathrm{NO}^{+}, \mathrm{CO}_{2}^{+}, \mathrm{N}_{2}^{+}$, and $\mathrm{CO}^{+}$densities decrease from solar minimum to solar maximum by factors of $114,66,20,9$, and 5 , respectively. The largest decreases are for those ions for which the most important loss process is dissociative recombination over a wide altitude range. Since the electron densities increase with solar activity, the loss rates of molecular ions by dissociative recombination also increase with solar activity. Thus, from low to high solar activities the model ionospheres exhibit composition changes, not just increases in the ion densities. At high solar activity the atomic ions make up a larger fraction of the total ion densities than at low solar activity, and the effect is greater at high altitudes than near the electron density peak. A similar composition variation for the nightside ionosphere was noted by Grebowsky et al. 
[1993] from PV OIMS data during the reentry period of the PV spacecraft. On the nightside the atomic ion densities (and thus the electron densities) increase from low to high solar activity, owing to the increase in the day-to-night ion transport. Thus the molecular ions on the nightside also are depleted at high altitudes owing to greater loss by dissociative recombination. Near the molecular ion peak, where the major loss process for the dominant ions is dissociative recombination, the solar activity variations are reduced to the square root of the production rate (see equation (4)).

Although we do not have in situ data about the ion composition at low solar activity, there are numerous radio occultation profiles from PV and other spacecraft, including Venera 9 and 10 , that cover the entire solar cycle. In our model the overall electron density profile exhibits two peaks. The major electron density peak of $2.9 \times 10^{5} \mathrm{~cm}^{-3}$ occurs at $141 \mathrm{~km}$ in the low solar activity model; the electron density peak is a factor of $\sim 1.6$ larger, $\sim 4.7 \times 10^{5} \mathrm{~cm}^{-3}$ at $139 \mathrm{~km}$ in the high solar activity model. The main electron density peak was observed at solar maximum, solar minimum, and during the declining phase of solar activity by PV ORO measurements over the 14-year lifetime of the spacecraft. Cravens et al. [1981] reported an average peak electron density for $60^{\circ}$ solar zenith angle, as measured by the PV ORO, of $4.7 \times 10^{5} \mathrm{~cm}^{-3}$ at an altitude of $140-141 \mathrm{~km}$. The magnitude of the peak is in excellent agreement with our model, although the altitude of the measured peak is higher than that in our model by 1-2 $\mathrm{km}$. Cravens et al. also fitted the solar zenith angle dependences of the radio occultation measurements at high and low solar activities with Chapman models, and they found that the best fits were for subsolar peak electron densities of $5 \times 10^{5}$ and $7.4 \times 10^{5} \mathrm{~cm}^{-3}$ for low and high solar activities, respectively. The model electron density peaks computed by Cravens et al. at $60^{\circ} \mathrm{SZA}$ were in the range $(5-6.5) \times 10^{5} \mathrm{~cm}^{-3}$, depending on the assumed profiles for $T_{e}$ and the adopted value for $F_{10.7}$. The Venera 9 and 10 measurements, which were at low solar activity, showed peak densities at $60^{\circ} \mathrm{SZA}$ of $\sim 3.6 \times 10^{5} \mathrm{~cm}^{-3}$ [Aleksandrov et al., 1976], which are $\sim 25 \%$ larger than our values.

Kliore and Mullen [1989] analyzed $115 \mathrm{PV}$ radio occultation profiles and derived an equation for the dayside peak electron density as a function of solar activity and solar zenith angle $\chi$ :

$$
\begin{aligned}
& n_{e, \max }\left(F_{\text {euv }}, \chi\right)=(5.92 \pm 0.03) \times 10^{5} \\
& \quad \times\left(F_{\text {euv }} / 150\right)^{0376 \pm 0011}(\cos \chi)^{0511 \pm 0.012},
\end{aligned}
$$

where $F_{\text {euv }}$ is an estimated value of $F_{10.7}$ corrected to the orbital position of Venus. For a solar zenith angle of $60^{\circ}$ the peak electron densities computed from (5) are $3.2 \times 10^{5}$ and $4.6 \times 10^{5} \mathrm{~cm}^{-3}$, for solar minimum $\left(F_{10.7} \approx 75\right)$ and solar maximum $\left(F_{10} \approx 200\right)$, respectively. These values agree to. within $10 \%$ with our model values.
A study of the variation of electron density $n_{e}$ measured by the PV OETP with solar activity over the 27day solar rotation period by Elphic et al. [1984] showed that $n_{e}$ in the lower ionosphere varied approximately as $V_{\text {euv }}^{033}$. $V_{\text {euv }}$ is a solar flux proxy derived from the rate of photoelectron production on the PV Langmuir probe (OETP) when it was outside the atmosphere [e.g., Brace et al., 1988]. The exponent of the solar flux proxy, 0.33 , is quite close to the exponent, $\mathbf{0 . 3 7 6}$, proposed by Kliore and Mullen [1989] for longer-term solar variations.

Average vertical electron density profiles for the $55^{\circ}-$ $75^{\circ}$ solar zenith angle range from radio occultation data taken at high solar activity (1979 and 1980) and low solar activity (1984 and 1986) were presented by Kliore and Luhmann [1991]. The average peak density was less than $3 \times 10^{5} \mathrm{~cm}^{-3}$ at low solar activity and increased by a factor of $\sim 50 \%$ from low to high solar activity, in very close agreement with our model values. The peak, however, appears slightly higher at high solar activity than at low solar activity in the average profiles, but it is near $140 \mathrm{~km}$ for all solar zenith angles. The altitude of the main electron density peak in our model is determined by both the background atmosphere, which was taken from the VTSS3 model, and by the assumed electron temperature profile. The VTS3 model was derived from in situ densities measured by the PV ONMS and the OAD experiment at high solar activity. The neutral densities in the VTS3 low solar activity model are extrapolations from the high solar activity model and are therefore less certain than the high solar activity values. Our calculations indicate that the VTS3 low solar activity $\mathrm{CO}_{2}$ densities, which are larger than those at high solar activity below $\sim 170 \mathrm{~km}$, may require a downward adjustment.

Keatıng and Hsu [1993] analyzed atmospheric drag measurements from the Magellan spacecraft and showed that the mass density at 1600 hours local time is $(1.2-1.3) \times 10^{-14} \mathrm{~g} \mathrm{~cm}^{-3}$ in the $170-190 \mathrm{~km}$ range when $F_{10} 7$ was $110-145$. Our low solar activity model $\left(F_{10}=80\right)$ exhibits a mass density of $1.4 \times 10^{-14} \mathrm{~g} \mathrm{~cm}^{-3}$ at $180 \mathrm{~km}$. This may also indicate that the low solar activity mass densities are somewhat too large, although the dominant constituent is $\mathrm{O}$ at this altitude.

The altitude of the peak ion density, however, is also affected by the electron temperature profile adopted. The electron temperature $T_{e}$ is observed to depart from $T_{n}$ and increase rapidly near $140 \mathrm{~km}$ [e.g., Miller et al., 1980; Cravens et al., 1980]. The major loss process for the molecular ions in this region is dissociative recombination, and the rate coefficients for dissociative recombination are usually proportional to $T_{e}^{-05 \pm 02}$, so in general the ion densities become greater as $T_{e}$ increases. We have found that the altitude at which the ion temperature is assumed to begin to increase rapidly in the model strongly affects the altitude of the peak. The measured $T_{e}$ profiles allow significant uncertainty in this altitude. The model calculations of Cravens et al. [1980] show a slight departure from the neutral tem- 
peratures between 130 and $140 \mathrm{~km}$ and a large difference above $140 \mathrm{~km}$.

Kim et al. [1989] compared high and low solar activity photochemical equilibrium models of the total ion densities to 16 individual PV ORO profiles at solar maximum and 11 at solar minimum. The average solar zenith angle at high solar activity was $62^{\circ}$, and the average latitude was $41^{\circ}$; for the low solar activity measurements the average SZA was $67^{\circ}$, and the average latitude was $62^{\circ}$. The average measured peak electron density was $4.5 \times 10^{5} \mathrm{~cm}^{-3}$ near $140 \mathrm{~km}$ at high solar activity and $2.8 \times 10^{5} \mathrm{~cm}^{-3}$ near $139 \mathrm{~km}$ at low solar activity. Once again, the magnitudes of the measured electron density peaks are in excellent agreement with our values of $4.7 \times 10^{5}$ and $2.9 \times 10^{5} \mathrm{~cm}^{-3}$, but the altitudes of the peaks are slightly different. The standard deviations about the average measured peak altitudes were only 0.22 and $0.15 \mathrm{~km}$ at high and low solar activities, respectively, so different peak shapes or altitudes should not have a large effect on the average peak altitudes or densities in their analysis. Although Kim et al. computed density profiles for six ions, only the total electron densities were reported. The computed maximum electron densities were $4 \times 10^{5}$ and $6 \times 10^{5} \mathrm{~cm}^{-3}$ in their low and high solar activity models, respectively. These values are larger than ours by $\sim 30 \%$; nonetheless, the predicted solar cycle variation of a factor of 1.5 is similar to ours. Their model electron density peak altitude of $137 \mathrm{~km}$ for solar maximum is even lower than ours, but the discrepancy may be due to the use of different photoabsorption coefficients for $\mathrm{CO}_{2}$, or more likely, to the difference in the assumed $T_{e}$ profiles. Above $160 \mathrm{~km}, \mathrm{Kim}$ et al. [1989] achieved agreement with the low solar activity measured electron densities by adopting electron temperatures that were less than those measured at high solar activity. Their low solar activity $T_{e}$ profile was computed from

$$
T_{e}=T_{n}+\left(T_{e}^{*}-T_{n}\right) / 2.5
$$

where $T_{e}{ }^{*}$ is the electron temperature from the empirical model of Theis et al. [1984].

Because the PV orbiter penetrated below $145 \mathrm{~km}$ only rarely, the peak was not sampled often by in situ instruments. Median ORPA ion densities at $155 \mathrm{~km}$ were reported by Miller et al. [1984a]. For a solar zenith angle bin $65^{\circ} \pm 5^{\circ} \mathrm{SZA}$ the median density total ion density was $2.2 \times 10^{5} \mathrm{~cm}^{-3}$. The total average electron density measured by the OETP in the 150-155 km range at $55^{\circ} \mathrm{SZA}$ was $2.48 \times 10^{5} \mathrm{~cm}^{-3}$ [Theis et al., 1984]. These values are smaller than our high solar activity total ion density at $155 \mathrm{~km}$ of $2.9 \times 10^{5} \mathrm{~cm}^{-3}$ but are in acceptable agreement.

A high-altitude peak in the $\mathrm{O}^{+}$density profile is predicted by our model at both solar activities but is apparent as a shoulder in the total electron density profile only at solar maximum, with a value of $\sim 1.7 \times 10^{5}$ $\mathrm{cm}^{-3}$ at $195 \mathrm{~km}$. At solar minimum the $\mathrm{O}^{+}$peak density is $2.6 \times 10^{4} \mathrm{~cm}^{-3}$ at $213 \mathrm{~km}$, and the electron den- sity profile shows only a change in slope, rather than a shoulder. The appearance of an $F_{2}$ peak in the $n_{e}$ profile at solar maximum but not at solar minimum is due to the greater mixing ratio of $O$ in the high solar activity model. The $O$ mixing ratio near $140 \mathrm{~km}$, as we have seen, is a factor of $\sim 2$ larger, and the $O$ density near 200 $\mathrm{km}$ is a factor of 2.8 larger at high solar activity than at low solar activity. The presente of a secondary peak or "ledge" has, however, been interpreted as evidence for magnetization of the ionosphere [e.g., Shinagawa et al., 1991; Woo and Kloore, 1991; Cravens, 1992; Cravens et al., 1997]. Our model suggests that if the actual $O$ density profiles are similar to those of the VTS3 model at equatorial latitudes, an $F_{2}$ peak should be visible at solar maximum and therefore caution should be exercised before the presence of the peak is interpreted as evidence for magnetization of the ionosphere, especially at solar maximum.

A review of the published radio occultation profiles, however, shows that a visible shoulder in this altitude region at high solar activity is seen only occasionally [Kliore and Mullen, 1989; Kloore and Luhmann, 1991]. The PV ORO profiles were, however, mostly at higher latitudes, where the $O$ mixing ratios may be smaller. If not, the VTS3 O mixing ratios may be too large at high solar activity. In the VIRA model, which applies to $F_{10}{ }_{7}=150$, the mixing ratio of $O$ near the ion peak, $\sim \mathbf{1 7 \%}$ [Keating et al, 1985], is similar to our high solar activity of $20 \%$. A study of the variation of the intensity of the O 1304- $\AA$ multiplet over the dayside from PV OUVS images suggests that $O$ brightnesses at latitudes greater than $30^{\circ}$ in the morning sector were much lower than in the evening sector, and that at the high-latitude morning terminator the $\mathrm{O}$ mixing ratios are up to a factor of 2.5 lower than those at the evening terminator [Alexander et al., 1993]. This asymmetry is not reflected in the VTS3 model. Our study is based on the VTS3 model at low latitudes, where little asymmetry in the 1304- $\AA$ emission was observed. Alexander et al. [1993], however, showed also that the column brightnesses inferred from the PV OUVS measurements at equatorial latitudes near 1600 hours local time were up to $20 \%$ lower than those computed from the VTS3 model, indicating that the column densities of $O$ were smaller than the integrated VTS3 $O$ density profiles, even at equatorial latitudes. Because of the uncertainty in the OUVS calibration, however, this could not be stated unequivocally.

In addition, the three-dimensional model of Mengel et al. [1989] showed that horizontal transport greatly affects the neutral density distributions. In particular, variations are produced in the neutral densities that are not reflected in the VTS3 model. Their derived high solar activity $O$ density at $170 \mathrm{~km}$ for their standard equatorial model with a thermospheric superrotation period of 6 days is less than half that of the VTS 3 model at 1600 hours local time. As discussed previously, the Magellan drag data analyzed by Keating and Hsu [1993] 
also showed that the low solar activity $O$ density profiles may be overestimates.

A comparison of our model electron densities at 200 and $300 \mathrm{~km}$ to in situ and remote-sensing measurements also shows a discrepancy. Our model densities at 200 and $300 \mathrm{~km}$ at high solar activities are $\sim 1.7 \times 10^{5}$ and $7.6 \times 10^{4} \mathrm{~cm}^{-3}$, respectively. The average ORO profiles presented by Brace and Kliore [1991] show electron densities at these altitudes of $\sim 1 \times 10^{5}$ and $4 \times 10^{4} \mathrm{~cm}^{-3}$, respectively. The $n_{e}$ model of Theis et al. [1984] shows even smaller densities of $8.95 \times 10^{4} \mathrm{~cm}^{-3}$ at $200 \mathrm{~km}$ and $3.42 \times 10^{4}$ at $300 \mathrm{~km}$. Since the major ion at this altitude is $\mathrm{O}^{+}$and the $F_{2}$ peak is near $200 \mathrm{~km}$, this may be further evidence that the $O$ density profiles in the VTS3 models are overestimates. Our high solar activity topside $\mathrm{O}^{+}$densities at both altitudes are too large by $\sim 60 \%$.

The only important loss process for $\mathrm{O}^{+}$at $200 \mathrm{~km}$ is

$$
\mathrm{O}^{+}+\mathrm{CO}_{2} \rightarrow \mathrm{O}_{2}^{+}+\mathrm{CO} \text {. }
$$

We adopted the rate coefficient for this reaction recommended by Anıcıch [1993], which is essentially equal to theoretical Langevin value of $1.1 \times 10^{-9} \mathrm{~cm}^{3} \mathrm{~s}^{-1}$. Since the Anucich [1993] evaluation, Viggiano et al. [1992] measured the rate coefficient and found it to be nearly collisional at low energies and to decrease slightly at temperatures above $800 \mathrm{~K}$. Use of the largest rate coefficient measured, $1.5 \times 10^{-9} \mathrm{~cm}^{3} \mathrm{~s}^{-1}$ [Smith and Futrell, 1972], reduces the $\mathrm{O}^{+}$peak density near $212 \mathrm{~km}$ by $\sim 10 \%$ and the electron density at $195 \mathrm{~km}$ by only $8 \%$. Thus adopting a larger rate coefficient for this reaction does not reduce the visible shoulder in the electron density profile.

At low solar activity there are no dayside in situ data, so we can only compare our model to the PV ORO profiles, where average values of $\sim 2.4 \times 10^{4}$ and $1.9 \times 10^{3} \mathrm{~cm}^{-3}$ at 200 and $300 \mathrm{~km}$, respectively, are reported [Brace and Kliore, 1991]. These values represent solar activity variations of a factor of 4 at 200 $\mathrm{km}$ and up to a factor of 20 at $300 \mathrm{~km}$. Our low solar activity model electron densities are $4.4 \times 10^{4}$ and $1.63 \times 10^{4} \mathrm{~cm}^{-3}$, at 200 and $300 \mathrm{~km}$, respectively, and the variations with solar activity are factors in the range $3.7-4.6$. The average ORO density profiles near 300 km appear very disturbed, with large oscillations, and the measured densities may be near the sensitivity of the instrument. Nonetheless, it is clear that the solar activity variation at $300 \mathrm{~km}$ is larger than our model indicates and may be an order of magnitude or so. If so, it is possible that the values for $T_{i}$ and/or $T_{e}$ that we have adopted, which are nearly the same as those at high solar activity, are too large at high altitudes. A reduction in the plasma temperatures would decrease the scale height of the ions on the topside, as Kim et al. [1989] showed. Mahajan and Mayr [1989] argue that the Venus low solar activity ionosphere is compressed by the interaction with the solar wind, which induces downward and horizontal transport of ions, resulting in a "photodynamical" ionopause. In this situation the $\mathrm{O}^{+}$ions on the topside take on the scale height of the underlying neutral $\mathrm{O}$, which is half that of the ion. Luhmann et al. [1987] suggested that the compressed nature of the ionosphere at solar minimum is evidence that the ionosphere is permeated by large-scale magnetic fields, which occurs when the ionospheric plasma pressure is not large enough to withstand the solar wind dynamic pressure. In any case, the interaction with the solar wind with the top of the atmosphere may produce a loss process for ions that we are unable to mimic in our one-dimensional models without magnetic fields.

It is noteworthy that the solar activity variations at the ion peak and above the ion peak on the dayside are very different, both in the measurements and in the models. The variation of the peak is $\sim 50-60 \%$ from solar minimum to solar maximum, a factor of $\sim 4$ at 200 $\mathrm{km}$ and a factor of 5-10 or more at $300 \mathrm{~km}$. The variations in the EUV fluxes, which produce the ions over most of the altitude range near and above the peak, are of the order of $2-3$. Thus there is a clear difference in the solar activity response at low altitudes and at high altitudes. This behavior is also seen on the nightside and has been interpreted as evidence that there are two different sources of ionization, ion transport from the dayside and electron precipitation, with very different solar activity dependences [e.g., Kliore et al., 1991; Knudsen et al., 1987]. We see here, however, that one cannot draw such a conclusion a priori. Indeed, the solar activity behavior of the molecular ions reverses sign from the peak to altitudes substantially above the peak. Thus the difference in solar activity response at high altitudes and low altitudes for both day and night may be caused by differences in the chemistry of atomic and molecular ions, in the underlying atmosphere, and/or in the plasma and neutral temperature profiles, rather than being indicative of differences in the sources of ionization.

\subsection{Minor Neutral Density Profiles}

We have also computed the density profiles of $\mathrm{NO}$, $\mathrm{N}\left({ }^{4} S\right), \mathrm{N}\left({ }^{2} D\right), \mathrm{N}\left({ }^{2} P\right), \mathrm{C}, \mathrm{O}\left({ }^{1} D\right)$, and $\mathrm{O}\left({ }^{1} S\right)$ from 90 $\mathrm{km}$ to the upper boundaries of the models, and the results are shown in Figures $6 a$ and $6 b$. 'The NO and N density profiles have been modeled previously by $R u s c h$ and Cravens [1979], by Fox [1982a], and by Gérard et al. [1988]. The NO density profiles show little solar activity variation. The densities at $105 \mathrm{~km}$ are $1.12 \times 10^{8}$ and $1.16 \times 10^{8} \mathrm{~cm}^{-3}$ at low and high solar activities, respectively. Between 120 and $200 \mathrm{~km}$ the $\mathrm{NO}$ densities are slightly anticorrelated with solar activity. This reflects an increase in the major loss processes at high solar activity. Densities at $125 \mathrm{~km}$ of $8.8 \times 10^{6}$ and $6.3 \times 10^{6}$ $\mathrm{cm}^{-3}$ are predicted for solar minimum and solar maximum, respectively. In the lower thermosphere, NO is produced mainly by the reaction 

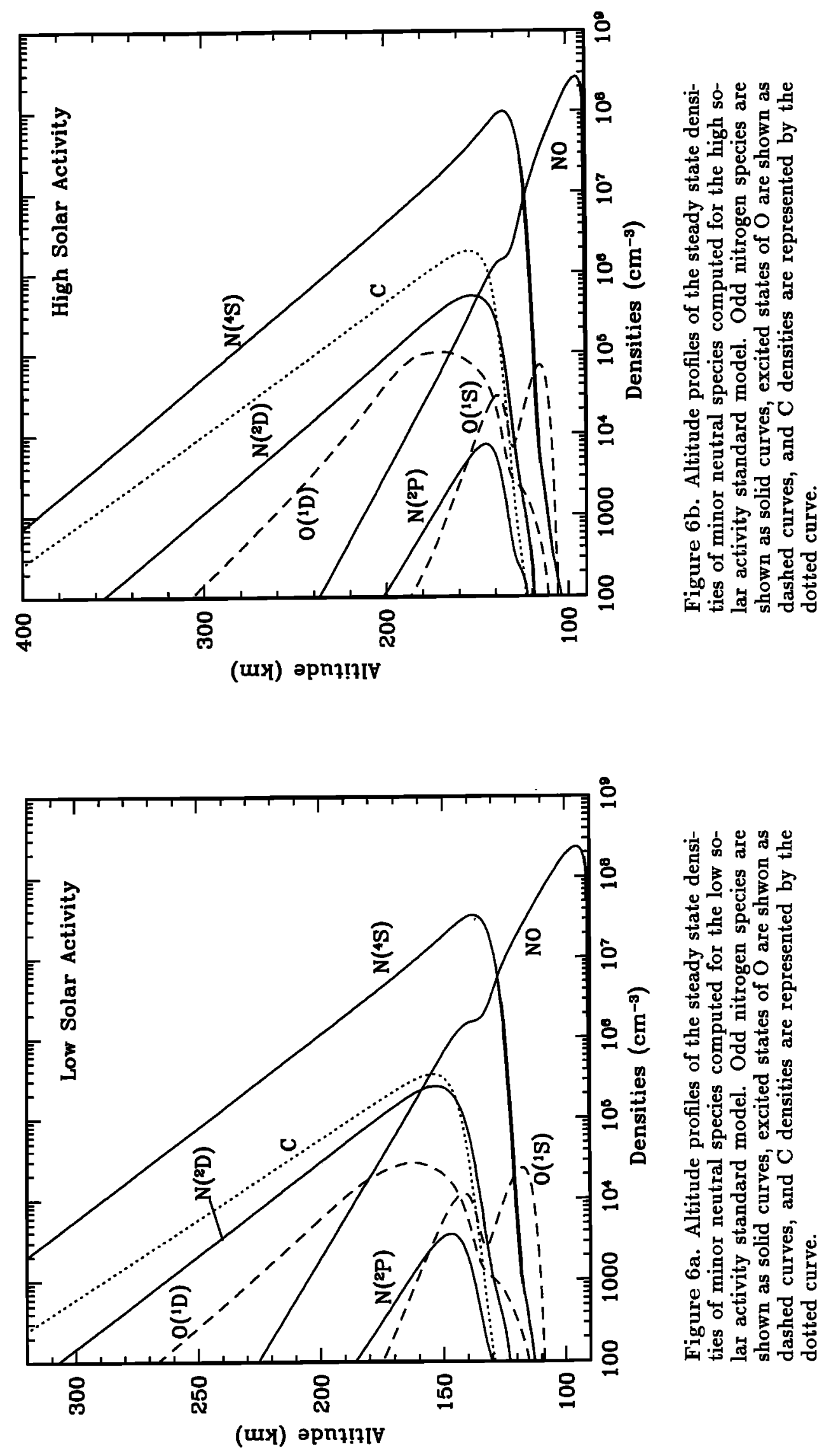


$$
\mathrm{N}\left({ }^{2} D\right)+\mathrm{CO}_{2} \rightarrow \mathrm{NO}+\mathrm{CO} .
$$

The analogous reaction of $\mathrm{N}\left({ }^{4} S\right)$ with $\mathrm{CO}_{2}$,

$$
\mathrm{N}\left({ }^{4} S\right)+\mathrm{CO}_{2} \rightarrow \mathrm{NO}+\mathrm{CO}
$$

becomes more important above $\sim 125 \mathrm{~km}$ if the rate coefficient is close to that assumed in the standard model, $1.7 \times 10^{-16} \mathrm{~cm}^{3} \mathrm{~s}^{-1}$, which is the upper limit reported by Brown and Winkler [1970] and Herron and Huie [1968]. As discussed in section 3 , it is possible that the rate coefficient for this reaction, which is spin forbidden, is much less than this value, and we discuss the effects of that assumption below. NO is destroyed mainly by the reaction with $\mathrm{N}$, reaction (R82) over most of the thermosphere, although charge transfer from $\mathrm{O}_{2}^{+}$to NO (reaction (R17)) becomes more important below $~ 120$ $125 \mathrm{~km}$. Both the $\mathrm{N}$ and $\mathrm{O}_{2}^{+}$densities increase with solar activity. The model NO densities below 95-100 km are highly dependent on the bottom boundary condition and should not be considered reliable. An arbitrary positive slope on the density profile, and thus a downward flux, was imposed at $90 \mathrm{~km}$ and varied in order to achieve a steady state solution to the continuity equation. The peak at $95 \mathrm{~km}$ may thus be an artifact of the boundary condition; it is possible that the NO densities continue to increase even below the bottom boundary at $90 \mathrm{~km}$.

The $\mathrm{N}\left({ }^{4} S\right)$ density profile exhibits a peak of $3.4 \times$ $10^{7} \mathrm{~cm}^{-3}$ at $138 \mathrm{~km}$ for solar minimum conditions and $1.0 \times 10^{8} \mathrm{~cm}^{-3}$ at $135 \mathrm{~km}$ for solar maximum conditions. This variation of about a factor of 3 is smaller than the variation of the major source mechanisms of a factor of $\sim 5$ near the peak. The major sources at the density peak are direct production by photodissociation and photoelectron impact dissociation of $\mathrm{N}_{2}$, and a few chemical reactions, including quenching of $\mathrm{N}\left({ }^{2} D\right)$ by $\mathrm{CO}$ and $O$ (reactions (R84) and (R86)), dissociative recombination of $\mathrm{NO}^{+}$(reaction (R122b)), and charge transfer of $\mathrm{N}^{+}$to $\mathrm{CO}_{2}$ (reaction (R28b)). The major loss processes are the reaction with NO (reaction(R82)) and

$$
\mathrm{O}_{2}^{+}+\mathrm{N} \rightarrow \mathrm{NO}^{+}+\mathrm{O}
$$

with the former dominating at low solar activity and the latter dominating at high solar activity. The increase in the density of $\mathrm{O}_{2}^{+}$with solar activity leads to an increase in the loss rate by (R15). This is largely responsible for the damping of the variation in the peak $\mathrm{N}$ density that is observed compared to that of the source functions.

If the rate coefficient for (R80), $k_{80}$, is much less than $10^{-16} \mathrm{~cm}^{3} \mathrm{~s}^{-1}$, the results differ considerably. We

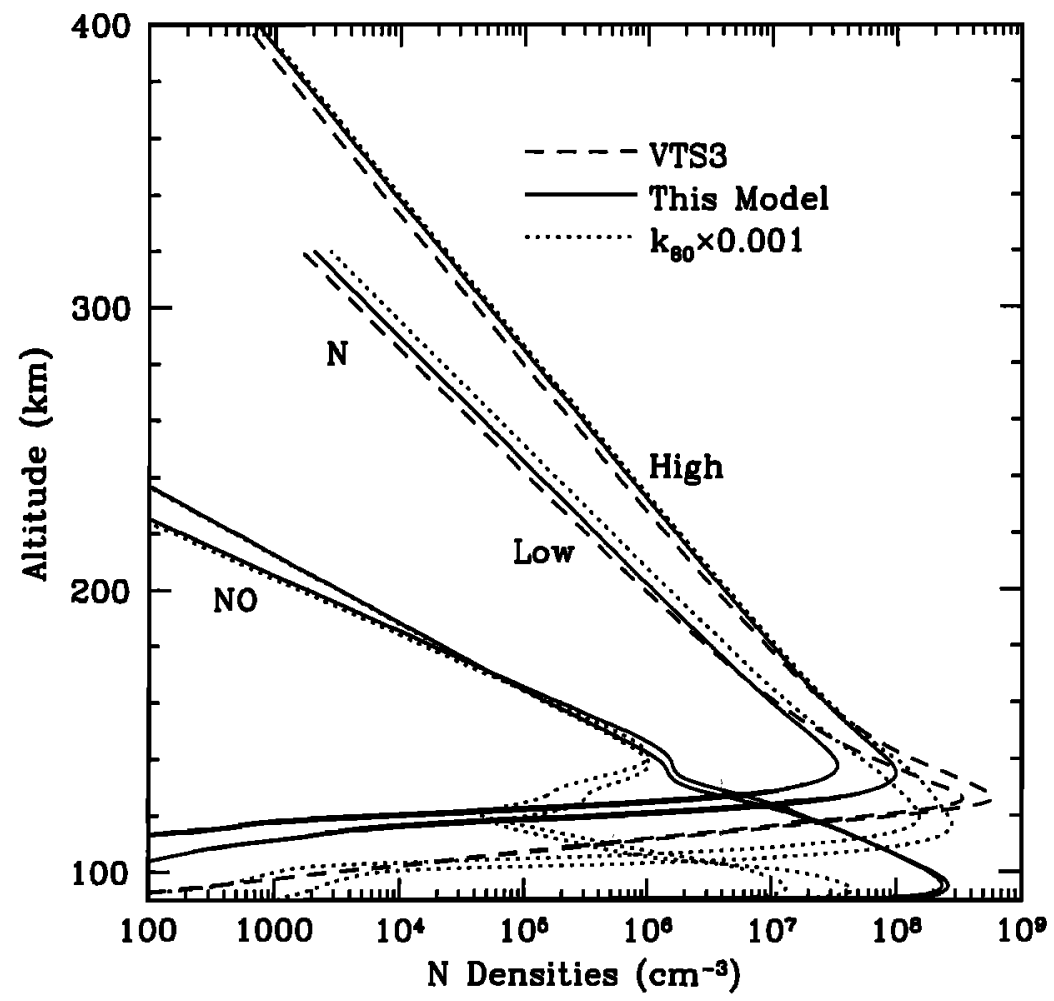

Figure 7. A comparison of atomic nitrogen densities computed from the standard $\left(k_{80}=\right.$ $\left.1.7 \times 10^{-16}\right)$ and alternative $\left(k_{80}=1.7 \times 10^{-19}\right)$ models with the VTS3 $\mathrm{N}$ densities for high and low solar activities. The solid curves are the $\mathrm{N}$ density profiles for the standard model, the dotted curves represent those for the alternative model, and the dashed curves are the VTS3 model densities from Hedin et al. [1983]. The NO density profiles for the standard model (solid curves) and the alternative model (dotted curves) are also shown for comparison. Little solar activity variation of the NO profiles is observed for either model. 
present our computed $\mathrm{N}$ and NO density profiles both for the standard model and for an alternative model in which $k_{80}$ is assumed to be a factor of 1000 smaller, $1.7 \times 10^{-19} \mathrm{~cm}^{3} \mathrm{~s}^{-1}$, and we compare the $\mathrm{N}$ density profiles to those input from the VTS3 model in Figure 7. The computed $\mathrm{N}$ densities for this model are slightly larger above $\sim 140 \mathrm{~km}$, and the density profiles peak at lower altitudes and larger values. For solar minimum the peak in the $\mathrm{N}$ density profile appears at 120 $\mathrm{km}$, with a value of $1.1 \times 10^{8} \mathrm{~cm}^{-3}$; at solar maximum the peak altitude is $117 \mathrm{~km}$, and the peak density is $2.8 \times 10^{8}$, factors of 2.8-3.5 larger than the standard model. The NO profile exhibits a complex structure, with an upper peak density of $\sim 1 \times 10^{6} \mathrm{~cm}^{-3}$ near 137 $\mathrm{km}$ for both models and a local minimum in the range $117-120 \mathrm{~km}$. The NO densities begin to increase again below this altitude. Near the bottom boundary of the model the NO densities are smaller than those in the standard model, with $95 \mathrm{~km}$ values of $1.3 \times 10^{7}$ and $4.4 \times 10^{7} \mathrm{~cm}^{-3}$, for low and high solar activities, respectively. If $k_{80}$ is assumed to be zero, near the bottom of the model, NO is greatly depleted, and the $\mathrm{N}$ densities peak near the boundary, with values at $100 \mathrm{~km}$ exceeding $10^{9} \mathrm{~cm}^{-3}$.

At $300 \mathrm{~km}$, for the standard models, the predicted $\mathrm{N}$ densities are $5.6 \times 10^{3}$ and $5.0 \times 10^{4} \mathrm{~cm}^{-3}$ for low and high solar activities, respectively. These values are $\sim 25 \%$ larger than the VTS3 model values of $4.5 \times 10^{3}$ and $4.0 \times 10^{4} \mathrm{~cm}^{-3}$. At $300 \mathrm{~km}$ in the alternative model the $\mathrm{N}$ densities are larger than both the standard model and the VTS3 model, but the difference is not great, and the high-altitude profiles cannot be used to estimate $k_{80}$. The differences in the various $\mathrm{N}$ profiles near and below the peak are, however, substantial. For the standard model the computed peak $\mathrm{N}\left({ }^{4} S\right)$ densities appear at higher altitudes with much smaller values than the VTS3 model for both high and low solar activities. The VTS3 model exhibits peak N densities of $2.4 \times 10^{8}$ and $5.7 \times 10^{8} \mathrm{~cm}^{-3}$ near $125 \mathrm{~km}$ for low and high solar activities, respectively. For the alternative model the peaks are lower, in the range $117-120 \mathrm{~km}$, with values of $1.54 \times 10^{8}$ and $2.8 \times 10^{8} \mathrm{~cm}^{-3}$, about a factor of 2 smaller than the VTS3 values. The VTS3 model is, however, based on in situ data from the PV orbiter, for which periapsis rarely fell below $140 \mathrm{~km}$, and the VTS3 $\mathrm{N}$ densities below $\sim 140 \mathrm{~km}$ were guided by the photochemical model of Rusch and Cravens [1979]. Our low-altitude model $\mathrm{N}$ density profiles therefore cannot be distinguished by comparing them to the VTS3 model values. A definitive measurement of $k_{80}$ would be helpful. Alternatively, in situ measurements of odd nitrogen densities in the lower thermosphere of Venus would help to constrain the value of $k_{80}$, although, unfortunately, no such measurements are planned for the near future.

The $\mathrm{N}\left({ }^{2} D\right)$ profiles exhibit peak densities of $2.5 \times 10^{5}$ and $5.2 \times 10^{5} \mathrm{~cm}^{-3}$ near $152 \mathrm{~km}$ at low and high solar activities, respectively. At those altitudes, $\mathrm{N}\left({ }^{2} D\right)$ is produced mainly in photodissociation and electron impact dissociation of $\mathrm{N}_{2}$, with substantial contributions from dissociative recombination of $\mathrm{NO}^{+}$, radiation from $\mathrm{N}\left({ }^{2} P\right)$, and quenching of $\mathrm{N}\left({ }^{2} P\right)$ by $\mathrm{O}$. The major loss processes are reaction with $\mathrm{CO}_{2}$ and quenching by $\mathrm{CO}$ and $O$, with a smaller contribution from quenching by electrons. The peak increase by a factor of 2 from solar minimum to solar maximum is slightly smaller than the increase in the magnitude of the production rates, which is a factor of 2.6. The damping is due to a larger increase in the rates for quenching by $\mathrm{CO}$ and $\mathrm{O}$, the mixing ratios of which are enhanced at higher solar activity.

The solar activity variation of the peak density of $\mathrm{N}\left({ }^{2} P\right)$, a factor of 2.0 , is similar to that of $\mathrm{N}\left({ }^{2} D\right)$. $\mathrm{N}\left({ }^{2} P\right)$ exhibits a peak density of $3.7 \times 10^{3} \mathrm{~cm}^{-3}$ near $147 \mathrm{~km}$ at low solar activity and at $7.5 \times 10^{3} \mathrm{~cm}^{-3}$ near $145 \mathrm{~km}$ at high solar activity. The major production mechanisms are photodissociation and electron impact dissociation of $\mathrm{N}_{2}$. The major loss process at the peak is radiation to $\mathrm{N}\left({ }^{2} D\right)$ and $\mathrm{N}\left({ }^{4} S\right)$, with significant destruction due to quenching, mostly by $\mathrm{O}$. The increase in the peak densities is damped slightly compared to that of the production rates, which increase by a factor of 2.3, owing to the increase in the rate of collisional deactivation by $O$, the mixing ratio of which increases with solar activity.

We can compare our computed high solar activity density profiles for odd nitrogen species with those predicted by Gérard et al. [1988]. Gérard et al. included the reaction of $\left.\mathrm{N}^{4} S\right)$ with $\mathrm{CO}_{2}$ in their odd nitrogen scheme but did not specify the rate coefficient that they used. Since their model showed a high-altitude peak for NO, we compare their predictions to our high solar activity alternative model. The $\mathrm{N}$ densities for the two models at $200 \mathrm{~km}$ are similar, but the peak densities are different. While Gérard et al. predict a peak density of $\mathrm{N}$ at $126 \mathrm{~km}$ of $6 \times 10^{7} \mathrm{~cm}^{-3}$, our $\mathrm{N}$ density peak is $9 \mathrm{~km}$ lower and a factor of 4.7 larger. The NO profiles peak at approximately the same altitude, near $137 \mathrm{~km}$, but the NO density at this altitude in the Gérard et al. model is $\sim 3 \times 10^{6} \mathrm{~cm}^{-3}$, a factor of 3 larger than our value. Below the peak their NO densities fall off to zero rapidly. In all of our models the densities of NO increase below $\sim 115 \mathrm{~km}$. The major source of NO in our model at low altitudes is reactions of $\mathrm{N}\left({ }^{2} D\right)$ with $\mathrm{CO}_{2}$ (reaction(R83)), and the major source of $\mathrm{N}\left({ }^{2} D\right)$ is dissociative recombination of $\mathrm{NO}^{+}$. Ions are produced near the bottom of our model by X-ray photoionization and the concomittant photoelectron impact ionization, and $\mathrm{NO}^{+}$is the terminal ion for many charge transfer and other ion-molecule reactions that take place in this region of high neutral densities. No other ion density is significant below $\sim 110 \mathrm{~km}$. The source of the differences with the Gérard et al. model, in addition to possible differences in the rate coefficient for reaction (R80), include many updated rate coefficients for other reactions. Gérard et al. did not consider harder $\mathrm{X}$ rays, but they influence the $\mathrm{N}$ and NO densities only below $\sim 120 \mathrm{~km}$.

The chemistry of atomic carbon in the Venus ther- 
mosphere has been discussed by Krasnopolsky [1982] and by Fox [1982b]. The major source of $\mathrm{C}$ is direct production by photodissociation of $\mathrm{CO}$, with a smaller contribution due to photoelectron impact dissociation. At high altitudes, $\mathrm{CO}^{+}$dissociative recombination (reaction (R119)) becomes dominant. The major loss process for $\mathrm{C}$ is reaction with $\mathrm{O}_{2}$. An $\mathrm{O}_{2}$ mixing ratio of $3 \times 10^{-3}$ was inferred by Paxton [1985] from the $\mathrm{C}$ density profiles determined from the limb profiles of the C I 1561and $1657-\AA$ emissions measured by the PV OUVS. For this $\mathrm{O}_{2}$ mixing ratio our model atomic carbon profile peaks near $154 \mathrm{~km}$ with a density of $3.5 \times 10^{5} \mathrm{~cm}^{-3}$ at low solar activity, and near $155 \mathrm{~km}$ with a density of $1.86 \times 10^{6} \mathrm{~cm}^{-3}$ at high solar activity. This factor of 5 increase in the $\mathrm{C}$ density is due to a combination of larger EUV fluxes and larger CO densities at high solar activity. As stated in section 4 , the $\mathrm{CO}$ mixing ratio is a factor of 1.75 larger at $140 \mathrm{~km}$ in the high solar activity model than in the low solar activity model. At higher altitudes the solar activity variations in the $\mathrm{C}$ densities are larger than those at the peak. The increase in $\mathbf{C}$ density from low to high solar activity is a factor of nearly 8 at $200 \mathrm{~km}$ and a factor of $\sim 16$ at 300 $\mathrm{km}$, mostly owing to the higher neutral temperatures and thus larger scale heights at high solar activity.

The peak atomic carbon density in the present model is a factor of 2.7 less than the value $5 \times 10^{6} \mathrm{~cm}^{-3}$ reported by Fox [1982b], partly because the low-resolution CO photodissociation cross sections of Cook et al. [1965], which were used in that model, have been substituted by the high-resolution cross sections constructed by Fox and Black [1989] to account for the discrete nature of the absorption in the $889-1118 \AA$ region. In this wavelength region, dissociation occurs by line absorption into predissociating states, and Fox and Black found that the total photodissociation rate was reduced when these cross sections were used. In addition, the rate coefficient for destruction of $\mathrm{C}$ by reaction with $\mathrm{O}_{2}$ (reaction (R102)) at $300 \mathrm{~K}$ has been revised upward from $3.3 \times 10^{-11}$ to $4.9 \times 10^{-11} \mathrm{~cm}^{3} \mathrm{~s}^{-1}$ [Chastanng et al, 2000]. Thus the model $\mathrm{C}$ densities are probably no longer compatible with the measurements of the ultraviolet lines of $\mathrm{C}$ by the PV OUVS, and the thermospheric $\mathrm{O}_{2}$ mixing ratio may be less than the adopted value of $3 \times 10^{-3}$ by a factor of $\sim 3$. If so, the rate coefficient for charge transfer of $\mathrm{O}^{+}$to $\mathrm{C}$ inferred here, $1 \times 10^{-10} \mathrm{~cm}^{3} \mathrm{~s}^{-1}$, may be too large by a factor of $\sim 3$. A simultaneous reanalysis of the chemistry of $\mathrm{C}$, the $\mathrm{PV}$ OUVS atomic carbon emissions, and PV OIMS $\mathrm{C}^{+}$densities is probably warranted before definite conclusions can be drawn.

The density profiles of $O\left({ }^{1} D\right)$ and $O\left({ }^{1} S\right)$ are also shown in Figures $6 \mathrm{a}$ and $6 \mathrm{~b}$. The $O\left({ }^{1} D\right)$ density peak in the low solar activity model is $\sim 2.8 \times 10^{4} \mathrm{~cm}^{-3}$ at $162 \mathrm{~km}$, and that in the high solar activity model is $\sim 1.03 \times 10^{5} \mathrm{~cm}^{-3}$ at $169 \mathrm{~km}$. This factor of 3.7 enhancement from low to high solar activity is larger than that of the solar EUV fluxes. As Fox and Dalgarno [1981] showed previously, the major column-integrated source of $\mathrm{O}\left({ }^{1} D\right)$ is photodissociation of $\mathrm{CO}_{2}$, but since $\mathrm{O}\left({ }^{1} D\right)$ is efficiently quenched, near the density peak $(160-170 \mathrm{~km})$ the production is about half by dissociative recombination of $\mathrm{O}_{2}^{+}$, and the remainder is due about equally to photodissociation of $\mathrm{CO}_{2}$ and to radiation from $\mathrm{O}\left({ }^{1} S\right)$. The loss processes near the peak are $\sim 75 \%$ quenching, and the rest are by radiation to the ground state at $6300 / 6364 \AA$. Near $200 \mathrm{~km}$, dissociative recombination becomes the most important source, and the loss is mainly by radiation. The predicted integrated intensity of the $\mathrm{O} 6300-\AA$ red line is $1.12 \mathrm{kR}$ at low solar activity and $5.0 \mathrm{kR}$ at high solar activity. Fox and Dalgarno [1981] predicted an intensity of 0.73 $k R$ for a (pre-Pioneer Venus) low solar activity model of the Venus thermosphere. Fox [1992] predicted a red line emission rate of $5.6 \mathrm{kR}$ for a high solar activity model of Venus based on PV data. Since the most important source of $\mathrm{O}\left({ }^{1} D\right)$ is dissociative recombination of $\mathrm{O}_{2}^{+}$in the regions where quenching is not effective, the predicted emission rate depends on the assumed yields of the ground and excited metastable states of $O$ in the dissociative recombination of $\mathrm{O}_{2}^{+}$. We have used here the recently measured branching ratios for the energetically allowed channels (reactions (R120a)-(R120d)) obtained from an ion storage ring by Kella et al. [1997].

For $\mathrm{O}\left({ }^{1} S\right)$ the most important source is photodissociation of $\mathrm{CO}_{2}$; dissociative recombination of $\mathrm{O}_{2}^{+}$is unimportant, contributing only $\sim 6 \%$ of the total production at $140 \mathrm{~km}$ in both models. The $\mathrm{O}\left({ }^{1} S\right)$ density profile exhibits two peaks as shown previously by Fox and Dalgarno [1981] and LeCompte et al. [1989]. The upper peak is due to photodissociative excitation of $\mathrm{CO}_{2}$ by solar photons with wavelengths mostly less than $\sim 1170 \AA$, and the lower peak is due to dissociative excitation of $\mathrm{CO}_{2}$ by Lyman alpha photons, which penetrate deeper in the thermosphere. The peaks at high solar activity are predicted to be $7.0 \times 10^{4} \mathrm{~cm}^{-3}$ at 115 $\mathrm{km}$ and $2.9 \times 10^{4} \mathrm{~cm}^{-3}$ at $139 \mathrm{~km}$. At low solar activity the peak densities are $2.4 \times 10^{4} \mathrm{~cm}^{-3}$ at $118 \mathrm{~km}$ and $1.2 \times 10^{4} \mathrm{~cm}^{-3}$ at $142 \mathrm{~km}$. The variation of the upper peak is thus a factor of 2.4 , and that of the lower peak is a factor of 2.9 ; these values directly reflect the variation in Hinteregger solar fluxes in the EUV and at Lyman alpha, respectively. The integrated overhead intensities of the atomic oxygen 5577- $\AA$ green line, which arises from the transition $\mathrm{O}\left({ }^{1} S\right) \rightarrow \mathrm{O}\left({ }^{1} D\right)$, are 48 and $129 \mathrm{kR}$ for the low and high solar activity models, respectively. The transition from $O\left({ }^{1} S\right)$ to the ground $O\left({ }^{3} P\right)$ state gives rise to the emission at $2972 \AA$. The predicted overhead integrated intensities are $2.0 \mathrm{kR}$ at solar minimum and $5.5 \mathrm{kR}$ at solar maximum. Fox and Dalgarno [1981] predicted overhead intensities of 48 and $2.4 \mathrm{kR}$ for the green line and the $2972-\AA$ emission bands, respectively; these intensities are slightly larger than the low solar activity values reported here. The oxygen airglow intensities computed here are summarized in Table 6.

LeCompte et al. [1989] analyzed limb profiles of the 2972- $\AA$ emission measured by the PV OUVS during the early high solar activity portion of the mission. The 
Table 6. Integrated Overhead Intensities of O Airglow Emissions at Low and High Solar Activities

\begin{tabular}{cccc}
\hline & Wavelength, & \multicolumn{2}{c}{ Intensities, kR } \\
\cline { 3 - 4 } Transition & $\AA$ & Low & High \\
\hline $\mathrm{O}\left({ }^{1} D\right) \rightarrow \mathrm{O}\left({ }^{3} P\right)$ & 6300 & 1.12 & 5.0 \\
$\mathrm{O}\left({ }^{1} S\right) \rightarrow \mathrm{O}\left({ }^{1} D\right)$ & 5577 & 48. & 129. \\
$\mathrm{O}\left({ }^{1} S\right) \rightarrow \mathrm{O}\left({ }^{3} P\right)$ & 2972 & 2.0 & 5.5 \\
& & & \\
\hline
\end{tabular}

measured zenith intensity for orbit 187 , which was at a local time of 1230 for near equatorial latitudes, was $7 \mathrm{kR}$ compared to $9 \mathrm{kR}$ for their model intensity. Our predicted intensity of $4.7 \mathrm{kR}$ is for a solar zenith angle of $60^{\circ}$. The prediction is thus in reasonable agreement with the measurements. LeCompte et al. modeled the limb profiles and obtained fairly good agreement at high altitudes, but between 105 and $120 \mathrm{~km}$ their model emission rates were much larger than the measured values. They attributed this difference to the departure of the actual temperatures from the model values due to gravity waves and to the resulting enhanced quenching of $\mathrm{O}\left({ }^{1} S\right)$.

\section{Summary and Conclusions}

We have constructed models of the low and high solar activity thermospheres and ionospheres of Venus for equatorial latitudes and 1600 hours local time. Our computations have shown that the production rates of some of the ions $\left(\mathrm{CO}_{2}^{+}, \mathrm{N}_{2}^{+}\right.$, and $\left.\mathrm{NO}^{+}\right)$at their peaks vary with solar activity by a factor of $\sim 2.5$, approximately as the solar EUV fluxes. The solar activity variation of $\mathrm{O}^{+}$production, both in the ground and excited states, and that of $\mathrm{CO}^{+}$were found to be enhanced over that of the EUV solar fluxes, owing to the greater mixing ratios of the neutral species from which they are formed. The variation of the $\mathrm{He}^{+}$peak production is larger than that of other ions because the variation of the solar fluxes at wavelengths shortward of the ionization threshold, $504.8 \AA$, is larger than that of longer-wavelength solar photons. Since the $\mathrm{H}$ densities decrease with solar activity, the production rate peak of $\mathrm{H}^{+}$is also anticorrelated with solar activity. The production peak is also found higher in the atmosphere, as is typical for an ion formed from a light species in an atmosphere in which the primary absorber is much heavier.

The variations of the peak ion densities with solar activity are summarized in Table $5 . \mathrm{O}_{2}^{+}$is the major ion over the range $120-190 \mathrm{~km}$, and its peak density varies by a factor of $\sim 1.6$, approximately as the square root of the solar flux. At altitudes less than $120 \mathrm{~km}, \mathrm{NO}^{+}$ dominates. In regions of high neutral densities such as the lower thermosphere of Venus, ionization flows from species whose parent neutrals have high ionization potentials to those that have low ionization potentials. Since the ionization potential of NO is the lowest of any of the atmospheric species considered here, its only loss process is dissociative recombination, and it is the terminal ion in the lower ionosphere. Direct production by ionization of $\mathrm{NO}$ is not important, because $\mathrm{NO}^{+}$ is formed in many ion-molecule reactions in the thermospheres of the terrestrial planets. The $\mathrm{NO}^{+}$density profile is complex and exhibits two peaks, which reflect the altitude variations of the production processes.

Since they are destroyed mainly by reactions with neutrals rather than by dissociative recombination, both $\mathrm{CO}^{+}$and $\mathrm{N}_{2}^{+}$peak high in the atmosphere. Their densities increase with altitude as the neutral densities decrease. The solar activity variation in the $\mathrm{N}_{2}^{+}$peak is about a factor of 2 , which is slightly less than that of the solar fluxes, because the increase in the production mechanisms is countered by the increase in the neutral densities with which it reacts from low to high solar activity. The peak in the $\mathrm{CO}^{+}$profile, however, increases by a larger factor of 3.3 from low to high solar activity because the mixing ratio of $\mathrm{CO}$ is enhanced at solar maximum, and the total production rate increases faster than the total loss rate as the solar activity increases.

The peak densities of the atomic ions, $\mathrm{O}^{+}, \mathrm{N}^{+}$, and $\mathrm{C}^{+}$, increase by relatively large factors of $5.2,7.8$, and 18 from low to high solar activity because their parent neutrals, $\mathrm{O}, \mathrm{N}$, and $\mathrm{C}$, are photochemically produced and also exhibit large solar cycle variations. The $\mathrm{C}^{+}$ density solar activity variation is greatly amplified compared to that of the solar fluxes because $\mathrm{C}^{+}$is produced from $C$, which is itself enhanced because it is formed by photodissociation of $\mathrm{CO}$, the densities of which also exhibit large solar activity variations. The larger variations of the atomic ion densities compared to those of the molecular ions produce a composition variation, in which the ratio of atomic to molecular ions increases with solar activity. In fact, the increase in the densities of atomic ions and thus electron densities at higher altitudes leads to an inverse response of the molecular ions on the topside, because they are destroyed at high altitudes by dissociative recombination.

Since there are no daytime in situ data at low solar activity, only radio occultation electron density profiles are available to compare to our low solar activity model. Our predicted electron-density peak varies from $2.9 \times 10^{5}$ to $4.7 \times 10^{5} \mathrm{~cm}^{-3}$, from low to high solar activity, in good agreement with the PV ORO profiles. The high solar activity electron density peak also agrees well with the OETP measurements. The computed electron 
density peak is, however, at a higher altitude in the solar minimum model than in the solar maximum model, in contrast to the measured profiles. This may indicate that the low solar activity VTS3 $\mathrm{CO}_{2}$ densities are inaccurate below $\sim 160-170 \mathrm{~km}$. Alternatively, it could indicate an error in the adopted $T_{e}$ profiles, which begin to diverge from $T_{n}$ near the ion peak.

In our models the high solar activity $\mathrm{O}^{+}$profile exhibits an $F_{2}$ peak that appears as a visible shoulder in the electron density profile. Such a peak is only occasionally seen in the high solar activity radio occultation profiles. The PV ORO measurements were mostly, however, at higher latitudes. This may indicate either that the VTS3 O mixing ratio is too large at high solar activity or that it is lower at high latitudes than at low latitudes.

The model electron densities in the 200-300 km range are too high by $\sim 60 \%$. Our model also underestimates the solar activity variation of the electron density at 300 $\mathbf{k m}$, where the low solar activity electron densities are much larger in the model than the average ORO profile shows. Both of these phenomena may also indicate that the model $O$ densities or our assumed low solar activity ion and/or electron temperatures are too high. In addition, at high altitudes the ion densities will be reduced by day-to-night transport, which is known to take place and to produce the nightside ionosphere. This effect can be represented in a one-dimensional model by putting an upward flux boundary condition on the ions. Preliminary calculations, however, indicate that the magnitude of this effect is not sufficient to produce the reduction needed in the low solar activity model at $300 \mathrm{~km}$. It is also possible that the direct interaction of the solar wind with the ionosphere cannot be mimicked near the ionopause in a one-dimensional model that does not incorporate magnetic fields [e.g., Luhmann et al., 1987].

In any case, both the measured and model solar activity variations of the dayside electron density profile exhibit a strong altitude dependence, even though the source of ionization is photoionization and photoelectron impact ionization at all altitudes. The observed and measured variations range from a factor of 1.6 at the peak to about a factor of 4 at $200 \mathrm{~km}$, to factors in the range $5-20$ at $300 \mathrm{~km}$. This same behavior is observed on the nightside but has been interpreted as evidence that different sources of ionization operate at different altitudes. This conclusion is not justified unless the solar activity response of the underlying neutral atmosphere, of the neutral, ion and electron temperatures, and of the differences in the identity and chemistry of the constituent ions at each altitude are taken into account. Indeed, the altitude variation of the solar activity response of the nightside ionosphere mirrors that of the dayside ionosphere.

Among the minor neutrals, we have computed the density profiles of the odd nitrogen species $\mathrm{NO}, \mathrm{N}\left({ }^{4} S\right)$, $\mathrm{N}\left({ }^{2} D\right)$, and $\mathrm{N}\left({ }^{2} P\right)$. The predicted density profiles of $\mathrm{N}$ and NO depend on the assumed rate coefficient for the reaction of $\mathrm{N}\left({ }^{4} S\right)$ with $\mathrm{CO}_{2}$ (reaction(R80)), for which various upper limits have been measured. The computed topside $\mathrm{N}$ density profiles fit the VTS3 values only slightly better for values of $k_{80}$ near $10^{-16} \mathrm{~cm}^{3} \mathrm{~s}^{-1}$, and the $\mathrm{N}$ density profile exhibits a factor of 3 solar activity variation at its peak. The $\mathrm{N}$ density peak is, however, a factor of 3 larger and $20 \mathrm{~km}$ lower for an alternative model in which $k_{80}$ is assumed to be $10^{-19} \mathrm{~cm}^{3} \mathrm{~s}^{-1}$. For either case the NO density profile shows little solar activity variation, although the profile exhibits a local peak near $137 \mathrm{~km}$ in the alternative model. This contrasts with the profile for the standard model, in which the density profile shows only a small shoulder near 137 $\mathrm{km}$ and then continues to increase as the altitudes decreases.

Atomic carbon is predicted to peak near $155 \mathrm{~km}$, and a large variation of a factor of 5 is predicted from low to high solar activity. Because of revisions in cross sections and rate coefficients in the past few years, our computed $\mathrm{C}$ densities are smaller than previous models have predicted. A comparison of our model densities to those required to reproduce the C 1561 - and 1651- $\AA$ atomic carbon limb profiles measured by the PV OUVS suggests that the adopted $\mathrm{O}_{2}$ mixing ratio of $1 \times 10^{-3}$ is too large by a factor of $\sim 3$, and the assumed rate coefficient for the charge transfer reaction of $\mathrm{O}^{+}$to $\mathrm{C}$ (reaction(R39)), $1 \times 10^{-10} \mathrm{~cm}^{3} \mathrm{~s}^{-1}$, is also overestimated by a similar factor. A detailed study of the PV OUVS C emission intensities and the OIMS $\mathrm{C}^{+}$densities is probably warranted before more precise values are deduced for the $\mathrm{O}_{2}$ mixing ratio and for $k_{39}$.

From the solar minimum to the solar maximum model, the peak $O\left({ }^{1} D\right)$ densities increase by a factor of 3.7, and the integrated overhead intensity of the $O\left({ }^{1} D\right) \rightarrow$ $\mathrm{O}\left({ }^{3} P\right)$ red line at $6300 / 6364 \AA$ is predicted to be 1.1 $\mathrm{kR}$ at low solar activity and $5.0 \mathrm{kR}$ at high solar activity. The integrated overhead intensity of the $\mathrm{O}\left({ }^{1} S\right) \rightarrow$ $\mathrm{O}\left({ }^{1} D\right) 5577-\AA$ green line is $48 \mathrm{kR}$ in the solar minimum model and $129 \mathrm{kR}$ in the solar maximum model; the corresponding intensities of the $\mathrm{O}\left({ }^{1} S\right) \rightarrow \mathrm{O}\left({ }^{3} P\right)$ 2972$\AA$ line are 2.0 and $5.5 \mathrm{kR}$.

Acknowledgments. This work has been supported in part by NASA grant NAG5-6879 to the Research Foundation of the State University of New York at Stony Brook. The compilation of revised rate coefficients and cross sections has been also partly supported by NASA grant NAG54188 to Wright State University and by NSF grant AST9802007 to the Research Foundation of the State University of New York at Stony Brook.

Janet G. Luhmann thanks Richard E. Hartle and Stephen W. Bougher for their assistance in evaluating this paper.

\section{References}

Aleksandrov, Y. N., et al., Preliminary results of two-frequenc radioscopy of the daytime ionosphere of Venus from Venera 9 and Venera 10, Kossmich. Issled., 14, 819-823, 1976.

Alexander, M. J., A. I. F. Stewart, S. C. Solomon, and S. W. Bougher, Local time asymmetries in the Venus thermosphere, J. Geophys. Res., 98, 10,849-10,871, 1993. 
Alge, E., N. G. Adams, and D. Smith, Measurements of the dissociative recombination coefficients of $\mathrm{O}_{2}^{+}, \mathrm{NO}^{+}$and $\mathrm{NH}_{4}^{+}$in the temperature range 200-600K, J. Phys. B, At. Mol. Phys., 16, 1433-1444, 1983.

Angel, G., and J. Samson, Total photoionization cross sections of atomic oxygen from threshold to $44.3 \AA$, Phys. Rev. A, 38, 5578-5585, 1988.

Anicich, V. G., Evaluated bimolecular ion-molecule gas phase kinetics of positive ions for use in modelling planetary atmospheres, cometary comae, and interstellar clouds, $J$. Phys. Chem. Ref. Data, 22, 1469-1569, 1993.

Atkinson, R., and K. H. Welge, Temperature dependence of $\mathrm{O}\left({ }^{1} S\right)$ deactivation by $\mathrm{CO}_{2}, \mathrm{O}_{2}, \mathrm{~N}_{2}$, and Ar, J. Chem. Phys., 57, 3689-3693, 1972.

Atkinson, R., D. L. Baulch, R. A. Cox, R. F. Hampson Jr., J. A. Kerr, M. J. Rossi, and J. Troe, Evaluated kinetic and photochemical data for atmospheric chemistry, suppl. VI, J. Phys. Chem. Ref. Data, 26, 1329-1499, 1997.

Ayres, T., Evolution of the solar ionizing flux, J. Geophys. Res, 102, 1641-1651, 1997.

Banks, P. M., and G. Kockarts, Aeronomy, Academic Press, San Diego, Calif., 1973.

Bates, D. R., Theoretical considerations regarding some inelastic atomic collision processes of interest in aeronomy: Deactivation and charge transfer, Planet. Space Sci., 17, 363-368, 1989.

Baulch, R. A., et al., Evaluated kinetic data for combustion modeling, Suppl. 1, J. Phys. Chem. Ref. Data, 23, 8471033, 1994.

Berrington, K. A., and P. G. Burke, Effective collision strengths for forbidden transitions in $e^{-}-\mathrm{N}$ and $e^{-}$ O scattering, Planet. Space Sci., 29, 377-381, 1981.

Bischof, G., and F. Linder, Crossed beam study of $\mathrm{He}^{+}$ $\mathrm{O}_{2}$ charge transfer reactions in the collision energy range 0.5-200 eV, Z. Phys. D, 1, 303-320, 1986.

Brace, L. H., and A. J. Kliore, The structure of the Venus ionosphere, Space Sci. Rev., 55, 81-163, 1991.

Brace, L. H., and R. F. Theis, An extension of the VIRA electron-temperature and density models to include solarcycle variations, Adv. Space Res., 17(11), 181-190, 1995.

Brace, L. H., et al., The dynamic behavior of the Venus ionosphere in response to solar wind interactions, J. Geophys. Res., 85, 7663-7678, 1980.

Brace, L. H., R. F. Theis, and W. R. Hoegy, Ionospheric electron temperature at solar maximum, Adv. Space Res., 7(6), 99-106, 1987.

Brace, L. H., W. R. Hoegy, and R. F. Theis, Solar EUV measurements at Venus based on photoelectron emission from the Pioneer Venus Langmuir probe, J. Geophys. Res., 93, 7282-7296, 1988

Brinton, H. C., H. A. Taylor Jr., H. B. Niemann, H. G. Mayr, A. F. Nagy, T. E. Cravens, and D. F. Strobel, Venus nighttime hydrogen bulge, Geophys. Res. Lett., 7, 865$868,1980$.

Brown, R., and C. A. Winkler, The chemical behaviour of active nitrogen, Angew. Chem., 9, 181-254, 1970.

Capetananakis, F. P, F. Sonderman, S. Höser, and F. Stuhl, Temperature dependence of the quenching of $\mathrm{O}\left({ }^{1} S\right)$ by simple inorganic molecules, J. Geophys. Res., 98, 7883$3887,1993$.

Chan, W. F., G. Cooper, and C. E. Brion, Absolute optical oscillator strengths for discrete and continuum photoabsorption of molecular nitrogen (11-200 eV), Chem. Phys. 170, 81-89, 1993b.

Chan, W. F., G. Cooper, and C. E. Brion, Absolute optical oscillator strengths for the photoabsorption of molecular oxygen (5-30 eV) at high resolution, Chem. Phys., 170, $99-109,1993 \mathrm{c}$.

Chapman, S., The absorption and dissociative or ionizing effects of monochromatic radiation in and atmosphere of a rotating earth, Proc. Phys. Soc. London, 43, 26-45, 1931.

Chastaing, D., S. D. Le Picard, and I. R. Sims, Direct kinetic measurements on reactions of atomic carbon, $\mathrm{C}\left({ }^{3} P\right)$, with $\mathrm{O}_{2}$ and $\mathrm{NO}$ at temperatures down to $15 \mathrm{~K}, J$. Chem. Phys., 112, 8466-8469, 2000.

Chen, R. H., and A. F. Nagy, A comprehensive model of the Venus ionosphere, J. Geophys. Res., 83, 3871-3876, 1978. Clyne, M. A. A., and I. S. McDermid, Mass spectrometric determinations of the rates of elementary reactions of $\mathrm{NO}$ and of $\mathrm{NO}_{2}$ with ground state $\mathrm{N}$ atoms, J. Chem. Soc. Faraday Trans. 1, 71, 2189-2202, 1975.

Constantinides, E. R., J. H. Black, and A. Dalgarno, The photochemistry of $\mathrm{N}^{+}$ions, Geophys. Res. Lett., 61, 569$572,1979$.

Cook, G. R., P. H. Metzger, and M. Ogawa, Photoionization and absorption coefficients of $\mathrm{CO}$ in the 600 to $1000 \AA$ region, Can. J. Phys., 43, 1706-1721, 1965.

Cravens, T. E., Ionospheric models for Venus and Mars, in Venus and Mars: Atmospheres, Ioncspheres and Solar Wind Interaction, Geophys. Monogr. Ser., vol. 66), edited by J. G. Luhmann, M. Tatrallyay, and R. Pepin, pp. 277-288, AGU, Washington, D.C., 1992.

Cravens, T. E., T. I. Gombosi, J. Kozyra, A. F. Nagy, L. H. Brace, and W. C. Knudsen, Model calculations of the dayside ionosphere of Venus: Energetics, J. Geophys. Res., $85,7778-7786,1980$.

Cravens, T. E., A. J. Kliore, J. U. Kozyra, and A. F. Nagy, The ionospheric peak on the Venus dayside, J. Geophys. Res., 86, 11,323-11,329, 1981.

Cravens, T. E., H. Shinagawa, and J. G. Luhmann, Magnetohydrodynamic processes: Magnetic fields in the ionosphere of Venus, in Venus II, edited by S. W. Bougher, D. M. Hunten, and R. J. Phillips, pp. 61-93, Univ. of Ariz. Press, Tucson, 1997.

Dalgarno, A., A. E. reaction rate data, Rep. AFGL-TR 790067, Air Force Geophys. Lab., Bedford, Mass., 1979.

Dalgarno, A., and J. L. Fox, Ion chemistry in atmospheric and astrophysical plasmas, in Unimolecular and Bimolecular Reaction Dynamics, edited by C. Y. Ng, T. Baer, and I. Powis, pp. 1-85, John Wiley, New York, 1994.

Davidson, D. F., and R. K. Hanson, High temperature reaction rate coefficients derived from $\mathrm{N}$-atom ARAS measurements and excimer photolysis of NO, Int. J. Chem. Kinet., 22, 843-861, 1990.

DeMore, W. B., S. P. Sander, D. M. Golden, R. F. Hampson, M. J. Kurylo, C. J. Howard, A. R. Ravishankara, C. E. Kolb, and M. J. Molina, Chemical kinetics and photochemical data for use in stratospheric modeling, evaluation number 10, JPL Publ., 92-20, 240 pp., 1992.

DeMore, W. B., S. P. Sander, D. M. Golden, R. F. Hampson, M. J. Kurylo, C. J. Howard, A. R. Ravishankara, C. E. Kolb, and M. J. Molina, Chemical kinetics and photochemical data for use in stratospheric modeling, evaluation number 10, JPL Publ., 94-26, 284 pp., 1994.

Dobe, Z., A. F. Nagy, L. H. Brace, R. F. Theis, and C. T. Russell, Energetics of the dayside ionosphere of Venus, Geophys. Res. Lett., 20, 1523-1526, 1993.

Donahue, T. M., D. H. Grinspoon, R. E. Hartle, and R. R. Hodges, Ion/neutral escape of hydrogen and deuterium: Evolution of water, in Venus $I I$, edited by S. W. Bougher, D. M. Hunten, and R. J. Phillips, pp. 385-414, Univ. of Ariz. Press, Tucson, 1997.

Dotan, I., and W. Lindinger, Energy dependences of the reactions of $\mathrm{Ar}^{+}$with $\mathrm{H}_{2}, \mathrm{~N}_{2}, \mathrm{CO}, \mathrm{O}_{2}, \mathrm{CO}_{2}, \mathrm{~N}_{2} \mathrm{O}$, and COS, J. Chem. Phys., 76, 4972-4977, 1982.

Dotan, I., and A. A. Viggiano, Rate constants for the reactions of $\mathrm{O}^{+}$with $\mathrm{NO}$ as a function of temperature (300$1400 \mathrm{~K})$, J. Chem. Phys., 110, 4730-4733, 1999. 
Dotan, I., P. M. Hierl, R. A. Morris, and A. A. Viggiano, Rate constants for the reactions of $\mathrm{N}^{+}$and $\mathrm{N}_{2}^{+}$with $\mathrm{O}_{2}$ as a function of temperature $(300-1800 \mathrm{~K})$, Int. J. Mass Spectrom. Ion Proc., 167/168, 223-230, 1997.

Dotan I., A. J. Midey, and A. A. Viggiano, Rate constants for the reactions of $\mathrm{Ar}^{+}$with $\mathrm{CO}_{2}$ and $\mathrm{SO}_{2}$ as a function of temperature (300-1500K), J. Am. Soc. Mass Spectrom., 10, 815-820, 1999.

Dotan, I., A. J. Midey, and A. A. Viggiano, Kinetics of the reactions of $\mathrm{N}_{2}^{+}$with $\mathrm{CO}_{2}$ and $\mathrm{SO}_{2}$ from $300-1400 \mathrm{~K}, J$. Chem. Phys., 113, 1732-1737, 2000.

Duff, J. W., and R. D. Sharma, Quasiclassical trajectory studies of the $\mathrm{N}\left({ }^{4} S\right)+\mathrm{NO} \rightarrow \mathrm{N}_{2}\left(X^{1} \Sigma_{g}^{+}\right)+\mathrm{O}\left({ }^{3} P\right)$, Geophys. Res. Lett., 23, 2777-2780, 1996.

Elphic, R. C., L. H. Brace, R. F. Theis, and C. T. Russell, Venus dayside ionospheric conditions: Effects of ionospheri, magnetic fields and solar EUV flux, Geophys. Res. Lett., $11,124-127,1984$.

Fahey, D. W., I. Dotan, F. C. Fehesenfeld, D. L. Albritton, and L. A. Viehland, Energy dependence of the rate constant of the reaction $\mathrm{N}^{+}+\mathrm{NO}$ at collision energies 0.04 to $2.5 \mathrm{eV}$, J. Chem. Phys., 74, 3320-3323, 1981a.

Fahey, D. W., F. C. Fehsenfeld, and E. E. Ferguson, Rate constant for the reaction $\mathrm{C}^{+}+\mathrm{CO}_{2}$ at collision energies 0.04 to $2.5 \mathrm{eV}$, Geophys. Res. Lett., 8, 1115-1117, $1981 \mathrm{~b}$.

Fehsenfeld, F. C., and E. E. Ferguson, Thermal energy reaction rates constants for $\mathrm{H}^{+}$and $\mathrm{CO}^{+}$with $\mathrm{O}$ and $\mathrm{NO}$, J. Chem. Phys., 56, 3066-3070, 1972.

Fehsenfeld, F. C., D. B. Dunkin, and E. E. Ferguson, Rate constants for the reaction of $\mathrm{CO}_{2}^{+}$with $\mathrm{O}, \mathrm{O}_{2}$, and $\mathrm{NO}$; $\mathrm{N}_{2}^{+}$with $\mathrm{O}$ and $\mathrm{NO}$; and $\mathrm{O}_{2}^{+}$with $\mathrm{NO}$, Planet. Space Sci., $18,1267-1269,1970$.

Fell, C., J. Stienfeld, and S. Miller, Quenching of $\mathrm{N}\left({ }^{2} D\right)$ by $\mathrm{O}\left({ }^{3} P\right)$, J. Chem. Phys., 92, 4768-4777, 1990.

Ferguson, E. E., Rate constants of thermal energy binary ion-molecule reactions of aeronomic interest, At. Data Nucl. Data Tables, 12, 159-178, 1973.

Ferguson, E. E., J. M. Van Doren, A. A. Viggiano, and R. A. Morris, Internal and translational energy effects on the charge-transfer reaction of $\mathrm{CO}_{2}^{+}$with $\mathrm{O}_{2}$, Int. J. Mass. Spectrom. Ion Proc., 117, 261-282, 1992.

Fernandez, A., A. Goumri, and A. Fontijn, Kinetics of the reactions of $\mathrm{N}\left({ }^{4} S\right)$ atoms with $\mathrm{O}_{2}$ and $\mathrm{CO}_{2}$ over wide temperature ranges, J. Phys. Chem., 102, 168-172, 1998.

Fox, J. L., The chemistry of metastable species in the Venusian ionosphere, Icarus, 51, 248-260, 1982a.

Fox, J. L., Atomic carbon in the atmosphere of Venus, $J$. Geophys. Res., 87, 9211-9216, 1982b. (Correction, J. Geophys. Res., 90,11,106, 1985.)

Fox, J. L., Airglow and aurora in the atmospheres of Venus and Mars, in Venus and Mars: Atmospheres, Ionospheres and Solar Wind Interaction, Geophys. Monogr. Ser., vol. 66), edited by J. G. Luhmann, M. Tatrallyay, and R. Pepin, pp. 191-222, AGU, Washington, D.C., 1992.

Fox, J. L., The production and escape of nitrogen atoms on Mars, J. Geophys. Res., 98, 3297-3310, 1993.

Fox, J. L., Rate coefficient for the reaction N + NO, J. Geophys. Res., 99, 6273-6276, 1994. (Correction, J. Geophys. Res., 101, 7987, 1996.)

Fox, J. L., and J. H. Black, Photodissociation of CO in the thermosphere of Venus, Geophys. Res. Lett., 16, 291-294, 1989.

Fox, J. L., and A. Dalgarno, Ionization, luminosity, and heating of the upper atmosphere of Venus, J. Geophys. Res., 86, 629-639, 1981.

Fox, J. L., and A. J. Kliore, Ionosphere: Solar activity variations, in Venus $I$, edited by S. Bougher, D. Hunten, and R. Phillips, pp. 161-188, Univ. of Ariz. Press, Tucson, 1997.
Fox, J. L., and G. A. Victor, $\mathrm{O}^{++}$in the Venusian ionosphere, J. Geophys. Res., 86, 2438-2441, 1981.

Fox, J. L,, P. Zhou, and S. W. Bougher, The thermosphere/ ionosphere of Mars at high and low solar activities, $A d v$. Space Res., 17(11), 203-218, 1996.

Frederick, J. E., and D. W. Rusch, On the chemistry of metastable atomic nitrogen in the $F$ region deduced from simultaneous satellite measurements of the $5200 \AA$ airglow and atmospheric composition, J. Geophys. Res., 82, 3509-3517, 1977.

Froese-Fischer, C., and H. P. Saha, Multiconfiguration Hartree-Fock results with Breit-Pauli corrections for forbidden transitions in the $2 \mathrm{p}^{4}$ configuration, Phys. Rev. A, 28, 3169-3178, 1983.

Frost, M. J., S. Kato, V. M. Bierbaum, and S. R. Leone, Reactions of $\mathrm{N}_{2}^{+}(v)$ with $\mathrm{CO}$ and $\mathrm{NO}$ at thermal energy, Chem. Phys., 231, 145-153, 1998.

Gérard J.-C., E. J. Deneye, and M. Lerho, Sources and distribution of odd nitrogen in the Venus daytime thermosphere, Icarus, 75, 171-184, 1988.

Gerlich, D., Inhomogeneous RF fields: A versatile tool for the study of processes with slow ions, in State-Selected and State-to-State Ion-Molecule Reaction Dynamics, Part 1, Experiment, edited by C.-Y. $\mathrm{Ng}$ and M. Baer, Adv. Chem. Phys. Ser., vol. LXXXII, John Wiley, New York, 1992.

Glosik, J., A. B. Rakshit, N. D. Twiddy, N. G. Adams, and D. Smith, Measurement of the rates of reaction of the ground and metastable excited states of $\mathrm{O}_{2}^{+}, \mathrm{NO}^{+}$and $\mathrm{O}^{+}$with atmospheric gases at thermal energy, J. Phys. B, At. Mol. Phys., 11, 3365-3379, 1978.

Goldan, P. D., A. L. Schmeltekopf, F. C. Fehsenfeld, H. I. Schiff, and E. E. Ferguson, Thermal energy ion-molecule reaction rates, II, Some reactions of ionospheric interest, J. Chem. Phys., 44, 4095-4013, 1966.

Gougousi, T., M. F. Golde, and R. Johnsen, Electron-ion recombination rate coefficient measurements in a flowing afterglow plasma, Chem. Phys. Lett., 265, 399-403, 1997.

Grebowsky, J. M., R. E. Hartle, J. Kar, P. A. Cloutier, H. A. Taylor, and L. H. Brace, Ion measurements during Pioneer Venus re-entry: Implications for solar cycle variation of ion composition and dynamics, Geophys. Res. Lett., 20, 2735-2738, 1993.

Grebowsky, J. M., W. T. Kasprzak, R. E. Hartle, and T. M. Donahue, A new look at Venus thermosphere $\mathrm{H}$ distribution, Adv. Space Res., 17(11), 191-195, 1995.

Hartle, R. E., T. M. Donahue, J. M. Grebowsky, and H. G. Mayr, Hydrogen and deuterium in the thermosphere of Venus: Solar cycle variations and escape, J. Geophys. Res., 101, 4525-2538, 1996.

Hedin, A. E., H. B. Niemann, W. T. Kasprzak, and A. Seiff, Global empirical model of the Venus thermosphere, $J$. Geophys. Res., 88, 73-83, 1983.

Herron, J. T., Evaluated chemical kinetic data for reactions of $\mathrm{N}\left({ }^{2} D\right), \mathrm{N}\left({ }^{2} P\right)$, and $\mathrm{N}_{2}\left(\mathrm{~A}^{3} \Sigma_{u}^{+}\right)$in the gas phase, $J$. Phys. Chem. Ref. Data, 28, 1453-1483, 1999.

Herron, J. T., and R. E. Huie, On the reaction of atomic nitrogen with carbon disxide, J. Phys. Chem., 72, 22352236, 1968

Hierl, P. M., I. Dotar, J. V. Seeley, J. M. Van Doren, R. A. Morris, and A. A. Viggiano, Rate constants for the reactioris of $\mathrm{O}^{+}$with $\mathrm{N}_{2}$ and $\mathrm{O}_{2}$ as a function of temperature (300 - 1800 K), J. Chem. Phys., 106, 3540-3544, 1997.

Huntress, W. T., J. K. Kim, and L. P. Thread, On the reaction of protons with methane, ammonia, water and oxygen, Chem. Phys. Lett., 29, 189-190, 1974.

Jamieson, M. J., J. M. Finch, R. S. Friedman, and A. Dalgarno, Collisional excitation of metastable oxygen $\mathrm{O}\left({ }^{1} D\right)$ 
atoms through the ${ }^{3} \Sigma_{u}^{+}$channel of $\mathrm{O}_{2}$, Planet. Space Sci., 40,1719-1721, 1992.

Johnsen, R., and M. A. Biondi, Laboratory measurements of the $\mathrm{O}^{+}\left({ }^{2} D\right)+\mathrm{N}_{2}$ and $\mathrm{O}^{+}\left({ }^{2} D\right)+\mathrm{O}_{2}$ reaction rate coefficients and their ionospheric implications, Geophys. Res. Lett., 7, 401-403, 1980.

Johnson, R. E., Comment on ion and electron temperatures in the Martian upper atmosphere, Geophys. Res. Lett., $5,989-992,1978$.

Keating, G. M., and N. C. Hsu, The Venus atmospheric response to solar cycle variations, Geophys. Res. Lett., 20, 2751-2754, 1993.

Keating, G. M., J. Y. Nicholson, and L. R. Lake, Venus upper atmosphere structure, J. Geophys. Res., 85, 7941$7956,1980$.

Keating, G. M., et al., Models of the Venus neutral upper atmosphere: Structure and composition, Adv. Space Res., 5(11), 117-171, 1985.

Kella, D. P., P. J. Johnson, H. B. Pedersen, L. VejbyChristensen, and L. H. Andersen, Branching ratios for dissociative recombination of ${ }^{15} \mathrm{~N}^{14} \mathrm{~N}$, Phys. Rev. Lett., 77, 2432-2435, 1996.

Kella, D., L. Vejby-Christenson, P. J. Johnson, H. B. Pedersen, and L. H. Andersen, The source of green light emission determined from a heavy-ion storage ring experiment, Science, 276, 1530-1533, 1997.

Kernahan, J. A., and P. H. L. Pang, Experimental determination of absolute A coefficients for 'forbidden' atomic oxygen lines, Can. J. Phys., 53, 455-458, 1975.

Kim, J., A. F. Nagy, T. E. Cravens, and A. J. Kliore, Solar cycle variations of the electron densities near the ionospheric peak of Venus, J. Geophys. Res., 94, 11,997$12,002,1989$.

Kim, Y. H., and J. L. Fox, The chemistry of hydrocarbon ions in the Jovian ionosphere, Icarus, 112, 310-325, 1994.

Kliore, A. J., and J. G. Luhmann, Solar cycle effects on the structure of the electron density profiles in the dayside ionosphere of Venus, J. Geophys. Res., 96, 21,281-21,289, 1991.

Kliore, A. J., and L. F. Mullen, Long term behavior of the main peak of the dayside ionosphere of Venus during solar cycle 21 and its implications on the effect of the solar cycle upon the electron temperature in the main peak region, J. Geophys. Res., 94, 13,339-13,351, 1989.

Kliore, A. J., G. S. Levy, D. L. Cain, G. Fjeldbo, and S. I. Rasool, Atmosphere and ionosphere of Venus from the Mariner 5 S-band radio occultation measurements, Science, 205, 99-102, 1967.

Kliore, A. J., I. R. Patel, A. F. Nagy, T. E. Cravens, and T. I. Gombosi, Initial nightside observations of the nightside ionosphere of Venus from Pioneer Venus orbiter radio occultation, Science, 203, 765-768, 1979.

Kliore, A. J., J. G. Luhmann, and M. H. G. Zhang, The effect of solar cycle on the maintenance of the nightside ionosphere of Venus, J. Geophys. Res., 96, 11,065-11,072, 1991.

Knudsen, W.C., The Verus ionosphere from in situ measurements, in Venus and Mars: Atmospheres, Ionospheres, and Solar Wind Interactions, Geophys. Monogr. Ser., vol. 66, edited by J. G. Luhmann, M. Tatrallyay, and R. Pepin, pp. 237-263, AGU, Washington, D.C., 1992.

Knudsen, W. C., K. L. Miller, K. Spenner, V. Novak, R. C. Whitten, and J. R. Spreiter, Thermal structure and energy influx to the day and nightside Venus ionosphere, Science, 205, 105-107, 1979.

Knudsen, W. C., A. J. Kliore, and R. C. Whitten, Solar cycle changes in the ionization sources of the nightside Venus ionosphere, J. Geophys. Res., 92, 13,391-13,398, 1987.
Krasnopolsky, V. A., Atomic carbon in the atmospheres of Mars and Venus, Kossmich. Issled., 20, 595-603, 1982.

LeCompte, M. A., L. J. Paxton, and A. I. F. Stewart, Analysis and interpretation of observations of airglow at $297 \mathrm{~nm}$ in the Venus thermosphere, J. Geophys. Res., 94, 208$216,1989$.

Lee, J. H., J. V. Michael, W. A. Payne, and L. Stief, Absolute rate of the reaction of $\mathrm{N}\left({ }^{4} S\right)$ with NO from 196-400 $\mathrm{K}$ with DF-RF and FP-RF techniques, J. Chem. Phys., 69, 3069-3076, 1978.

Lewis, B. R., and J. H. Carver, Temperature dependence of the carbon dioxide photoabsorption cross section between 1200 and $1970 \AA$, J. Quant. Spectrosc. Radiat. Transfer, 30, 297-309, 1983.

Li, X., Y.-L. Huang, G. D. Flesch, and C. Y. Ng, Absolute state-selected total cross sections for the ion-molecule reactions $\mathrm{O}^{+}\left({ }^{4} S,{ }^{2} D,{ }^{2} P\right)+\mathrm{H}_{2}\left(\mathrm{D}_{2}\right)$, J. Chem. Phys., 106, $564-571,1997 \mathrm{a}$.

Li, X., Y.-L. Huang, G. D. Flesch, and C. Y. Ng, A stateselected study of the ion-molecule reactions $\mathrm{O}^{+}\left({ }^{4} S,{ }^{2} D,{ }^{2} P\right)$ $+\mathrm{N}_{2}$, J. Chem. Phys., 106, 1373-1381, 1997b.

Lindackers, D., M. Burmeister, and P. Roth, High-temperature kinetics of the reaction $\mathrm{CN}+\mathrm{CO}_{2}$, Combust. Flame, $81,251-259,1990$.

Luhmann, J. G., C. T. Russell, F. L. Scarf, L. H. Brace, and W. C. Knudsen, Characteristics of Marslike limit of the Venus-solar wind interaction, J. Geophys. Res, 92, 8545-8557, 1987.

Mahajan, K. K., and H. G. Mayr, Venus ionopause at solar minimurn, Geophys. Res. Lett., 16, 1477-1480, 1989.

Mahajan, K. K., S. Gosh, R. Paul, and W. R. Hoegy, Variability of the dayside electron temperature at Venus, Geophys. Res. Lett., 21, 77-80, 1994.

Mauclaire, G., R. Derai, S. Fenistein, R. Marx, and R. Johnsen, Thermal energy charge transfer from $\mathrm{He}^{+}$to $\mathrm{O}_{2}$ : Kinetic energy, nature, and reactivity of the $\mathrm{O}^{+}$product ions, J. Chem. Phys., 70, 4023-4026, 1979.

McElroy, M. B., and M. C. McConnell, Atomic carbon in the atmospheres of Mars and Venus, J. Geophys. Res., 76, 6674-6690, 1971.

McFarland, M., D. L. Albritton, F. C. Fehesenfeld, E. E. Ferguson, and A. L. Schmeltekopf, Energy dependence and branching ratios of the $\mathrm{N}_{2}^{+}+\mathrm{O}$ reaction, J. Geophys. Res., 79, 2925-2926, 1974.

McLaughlin, B. M., and K. L. Bell, Electron-impact excitation of the fine-structure levels $\left(1 s^{2} 2 s^{2} 2 p^{3}{ }^{4} P_{3 / 2}^{o},{ }^{2} D_{5 / 2,3 / 2}^{o}\right.$, ${ }^{2} P_{3 / 2,1 / 2}^{o}$ ) of singly ionized atomic oxygen, J. Phys. B, At. Mol. Phys.,, 31, 4317-4329, 1998.

Mehr, F.J., and M. A. Biondi, Electron temperature dependence of recombination of $\mathrm{O}_{2}^{+}$and $\mathrm{N}_{2}^{+}$ions with electrons, Phys. Rev., 181, 264-270, 1969.

Mengel, J. G., D. R. Stevens-Rayburn, H. G. Mayr, and I. Harris, Non-linear three dimensional spectral model of the Venusian thermosphere with super-rotation, II, Temperature, composition, and winds, Planet. Space Sci., 37, $707-722,1989$.

Michael, J. V., and K. P. Lim, Rate constants for the $\mathrm{N}_{2} \mathrm{O}$ reaction system: Thermal decomposition of $\mathrm{N}_{2} \mathrm{O} ; \mathrm{N}+\mathrm{NO}$ $\rightarrow \mathrm{N}_{2}+\mathrm{O}$; and implications for $\mathrm{O}+\mathrm{N}_{2} \rightarrow \mathrm{NO}+\mathrm{N}, J$. Chem. Phys., 97, 3228-3234, 1992.

Midey, A. J., and A. A. Viggiano, Rate constants for the reaction of $\mathrm{Ar}^{+}$with $\mathrm{O}_{2}$ and $\mathrm{CO}$ as function of temperature from $300 \mathrm{~K}$ to $1400 \mathrm{~K}$ : Derivation of rotational and vibrational energy effects, J. Chem. Phys., 109, 5257-5263, 1998.

Midey, A. J., and A. A. Viggiano, Rate constants for the reaction of $\mathrm{O}_{2}^{+}$with $\mathrm{NO}$ from 300 to $1400 \mathrm{~K}, \mathrm{~J}$. Chem. Phys., 110, 10,746-10,748, 1999.

Miller, K. L., W. C. Knudsen, K. Spenner, R. C. Whit- 
ten, and V. Novak, Solar zenith angle dependence of ionospheric ion and electron temperatures and densities on Venus, J. Geophys. Res., 85, 7759-7764, 1980.

Miller, K. L., W.C. Knudsen, and K. Spenner, The dayside Venus ionosphere, Icarus, 57, 386-409, $1984 \mathrm{a}$.

Miller, T. M., R. E. Wetterskog, and J. F. Paulson, Temperature dependence of the ion-molecule reactions $\mathrm{N}^{+}+$ $\mathrm{CO}, \mathrm{C}^{+}+\mathrm{NO}$, and $\mathrm{C}^{+}, \mathrm{CO}^{+}, \mathrm{CO}_{2}^{+}+\mathrm{O}_{2}$ from $90-450 \mathrm{~K}$, J. Chem. Phys., 80, 4922-4925, $1984 \mathrm{~b}$.

Nagy, A. F., and T. E. Cravens, Ionosphere: Energetics, in Venus II, edited by S. W. Bougher, D. M. Hunten, and R. J. Phillips, pp. 189-224, Univ. of Ariz. Press, Tucson, 1997.

Nagy, A. F., T. E. Cravens, S. G. Smith, H. A. Taylor, and H. C. Brinton, Model calculations of the dayside ionosphere of Venus: Ionic composition, J. Geophys. Res., 85, 7795-7801, 1980.

Nagy, A. F., T. E. Cravens, and T. I. Gombosi, Basic theory and model calculations of the Venus ionosphere, in Venus, edited by D. M. Hunten et al., pp. 841-872, Univ. of Ariz. Press, Tucson, 1983.

Niemann, H. B., W. T. Kasprzak, A. E. Hedin, D. M. Hunten, and N. W. Spencer, Mass spectrometric measurements of the neutral gas composition of the thermosphere and exosphere of Venus, J. Geophys. Res., 85, 7817-7828, 1980.

O'Keefe, A., G. Mauclaire, D. Parent, and M. T. Bowers, Product energy disposal in the reaction of $\mathrm{N}^{+}\left({ }^{3} P\right)$ with $\mathrm{O}_{2}\left(X^{3} \Sigma\right)$, J. Chem. Phys., 84, 215-219, 1986.

Paxton, L. J., Pioneer Venus orbiter ultraviolet spectrometer limb observations: Analysis and interpretation of the 166- and 156-nm data, J. Geophys. Res., 90, 5089-5096, 1985.

Paxton, L. J., $\mathrm{CO}^{+}$and $\mathrm{N}_{2}^{+}$in the Venus ionosphere, $J$. Geophys. Res., 93, 8473-8482, 1988.

Perry, J. J., Y. H. Kim, J. L. Fox, and H. S. Porter, Chemistry of the Jovian auroral ionosphere, J. Geophys. Res., 104, 16,541-16,565, 1999.

Piper, L. G., The reactions of $\mathrm{N}\left({ }^{2} P\right)$ with $\mathrm{O}_{2}$ and $\mathrm{O}, J$. Chem. Phys., 98, 8560-8564, 1993.

Prasad, S. S., and W. T. Huntress Jr., A model for gas phase chemistry in interstellar clouds, I, The basic model, ibrary of chemical reactions, and chemistry among C, N, and O compounds, Astrophys. J. Suppl. Ser., 43, 1-35, 1980.

Rawlins, W. T., and F. Kaufman, The reaction of $\mathrm{CO}_{2}$ with active nitrogen, J. Chem. Phys., 64, 1128-1133, 1976.

Rawlins, W. T., M. E. Fraser, and S. M. Miller, Robvibrational excitation of nitric oxide in the reaction of $\mathrm{O}_{2}$ with atomic nitrogen, J. Phys. Chern., 93, 1097-1107, 1989.

Rosén, S., R. Peverall, M. Larsson, A. LePadellec, J. Semaniak, A. Larson, C. Stromholm, W. J. van der Zande, H. Danared, and G. H. Dunn, Absolute cross sections and final-state distributions for dissociative recombination and excitation of $\mathrm{CO}^{+}(v=0)$ using an ion storage ring, Phys. Rev. A, 57, 4462-4471, 1998.

Rusch, D. W., and T. E. Cravens, A model of the neutral and ion nitrogen chemistry in the dayside thermosphere, Geophys. Res. Lett., 6, 791-793, 1979.

Rusch, D. W., D. G. Torr, P. B. Hays, and J. C. G. Walker, The OII (7319-7330 $\AA$ ) dayglow, J. Geophys. Res., 82, 719-722, 1977.

Samson, J. A. R., and G. C. Angel, Single and doublephotoionization cross sections of atomic nitrogen from threshold to $31 \AA$, Phys. Rev. A, 42, 1307-1312, 1990 .

Samson, J. A. R., and P. N. Pareek, Absolute photoionization cross-sections of atomic oxygen, Phys. Rev. A, 31, 1470-1476, 1985.

Samson, J. A. R., Z. X. He, L. Yin, and G. N. Haddad,
Precision measurements of the absolute photoionization cross sections of He, J. Phys. B, 27, 887-898, 1994.

Schofield, K., Rate constants for the gaseous interactions of $\mathrm{O}\left(2^{1} D_{2}\right)$ and $\mathrm{O}\left(2^{1} S_{0}\right)$ : A critical evaluation, J. Photochem., 9, 55-68, 1978.

Scott, G. B., D. A. Fairley, C. G. Freeman, M. J. McEwan, P. Spanel, and D. Smith, Gas phase reactions of some positive ions with atomic and molecular hydrogen at 300 K, J. Chem. Phys., 106, 3982-3987, 1997.

Scott, G. B. I., D. A. Fairley, C. G. Freeman, M. J. McEwan, and V. J. Anicich, Gas-phase reactions of some positive ions with atomic and molecular nitrogen, J. Chem. Phys., 109, 9010-9014, 1998.

Scott, G. B. I., D. A. Fairley, D. B. Milligan, C. G. Freeman, and M. J. McEwan, Gas phase reactions of some positive ions with atomic and molecular oxygen and nitric oxide at $300 \mathrm{~K}, J$. Phys. Chem. A, 103, 7470-7473, 1999.

Seaton, M. J., and D. E. Osterbrock, Relative intensities in gaseous nebulae, Astrophys. J., 125, 66-83, 1957.

Shaw, D. A., D. M. P. Holland, M. A. Hayes, M. A. McDonald, A. Hopkirk, and S. M. McSweeney, A study of the absolute photoabsorption, photoionization and photodissociaion cross sections and the photoionization quantum efficiency of carbon dioxide from the ionization threshold to $345 \AA$, Chem. Phys., 198, 381-396, 1995.

Shihira, Y., T. Suzuki, S. Unayama, H. Umemoto, and S. Tsunashima, Reactions of $\mathrm{N}\left(2^{2} D\right)$ and $\mathrm{N}\left(2^{2} P\right)$ with $\mathrm{O}_{2}$, J. Chem. Soc. Faradxy Trans., 90, 549-552, 1994.

Shinagawa, H., J. Kim, A. F. Nagy, and T. E. Cravens, A comprehensive magnetohydrodynamic model of the Venus ionosphere, J. Geophys. Res., 96, 11,083-11,095, 1991.

Siskind, D. E., and D. R. Rusch, Nitric oxide in the middle to upper thermosphere, J. Geophys. Res., 97, 3209-3217, 1992.

Smith, D. L., and J. H. Futrell, Ion-molecule reactions in the $\mathrm{CO}_{2} / \mathrm{H}_{2}$ system by ion cyctotron resonance, Int. J. Mass Spectrom. Ion Phys., 10, 405-418, 1972.

Stewart, A. I., D. E. Anderson, L. W. Esposito, and C. A. Barth, Ultraviolet spectroscopy: Initial results from the Pioneer Venus Orbiter, Science, 203, 777-779, 1979.

Streit, G. E., C. J. Howard, and A. L. Schmeltekopf, Temperature dependence of $\mathrm{O}\left({ }^{1} D\right)$ rate constants for reactions with $\mathrm{O}_{2}, \mathrm{~N}_{2}, \mathrm{CO}, \mathrm{O}_{3}$ and $\mathrm{H}_{2} \mathrm{O}, J$. Chem. Phys., 65, 47614764,1976 .

Stuhl, F., and K. H. Welge, Deactivation of $O\left({ }^{1} D\right)$ and $\mathrm{O}_{2}\left(b^{1} \Sigma_{g}^{+}\right)$, Can. J. Chem., 47, 1870-1871, 1969.

Sung, K. Y., and J. L. Fox, Electron impact cross sections for use in modeling the ionosphere/thermospheres of the Earth and planets, Eos Trans. $A G U, 81(48)$, Fall Meet. Suppl., F931, SA52A-11, 2000.

Taylor, H. A., H. C. Brinton, S. J. Bauer, R. E. Hartle, P. A. Cloutier, and R. E. Daniell, Global observations of the composition and dynamics of the ionosphere of Venus: Implications for the solar wind interaction, $J$. Geophys. Res., 85, 7765-7777, 1980.

Taylor, H. A., H. C. Brinton, H. B. Niemann, H. G. Mayr R. E. Hartle, A. Barnes, and J. Larson, Interannual and short term variations of the Venus nighttime hydrogen bulge, J. Geophys. Res., 89, 669-675, 1984.

Theis, R. G., and L. H. Brace, Solar cycle variations of electron density and temperature in the Venusian nightside ionosphere, Geophys. Res. Lett., 20, 2719-2722, 1993.

Theis, R. F., L. H. Brace, R. C. Elphic, and H. G. Mayr, New empirical models of the electron temperature and density in the Venus ionosphere with application to transterminator flow, J. Geophys. Res., 89, 1477-1488, 1984.

Torr, D. G., and M. R. Torr, Determination of the thermal rate coefficient, products and branching ratios for the re- 
action of $\mathrm{O}^{+}\left({ }^{2} D\right)$ with $\mathrm{N}_{2}, J$. Geophys. Res., 85, 783-787, 1980.

Torr, D. G., M. R. Torr, R. A. Ong, and H. A. Hinteregger, Ionization frequencies for major thermospheric constituents as a function of solar cycle 21, Geophys. Res. Lett., 6, 771-774, 1979.

Tsang, W., and R. F. Hampson, Chemical kinetic data base for combustion chemistry, part I, Methane and related compounds, J. Phys. Chem. Ref. Data, 15, 1087-1279, 1986 .

Uiterwaal, C. J. G. J., J. van Eck, and A. Niehaus, Stateselected ion-molecule reactions: Charge transfer and atomic rearrangement processes in thermal energy collisions of $\mathrm{H}_{2}^{+}(X ; v)+\mathrm{N}_{2}$ and of $\mathrm{N}_{2}^{+}(X, A ; v)+\mathrm{H}_{2}, J$. Chem. Phys., 102, 744-753, 1995.

Vejby-Christensen, L., D. Kella, H.B. Pedersen, and L.H. Andersen, Dissociative recombination of $\mathrm{NO}^{+}$, Phys. Rev. $A, 57,3627-3634,1998$.

Verner, D. A., and D. G. Yakovlev, Analytic fits for partial photoionization cross sections, Astron. Astrophys. Suppl. Ser., 109, 125-133, 1995.

Verner, D. A., G. J. Ferland, K. T. Korista, and D. G. Yakovlev, Atomic data for astrophysics, 2, New analytic fits for photoionization cross sections of atoms and ions, Astrophys. J., 465, 487-498, 1996.

Viggiano, A. A., R. A. Morris, and J. F. Paulson, Rate constant and branching fractions for the reaction of $\mathrm{O}^{+}\left({ }^{2} D\right.$, ${ }^{2} P$ ) with $\mathrm{CO}_{2}$, J. Chem. Phys., 93, 1483-1484, 1990.

Viggiano, A. A., R. A. Morris, J. M. Van Doren, and J. F. Paulson, Temperature, kinetic energy, and internal energy dependences of the rate constant and branching fraction for the reaction of $\mathrm{O}^{+}\left({ }^{4} S\right)$ with $\mathrm{CO}_{2}, J$. Chem. Phys., 270, 270-274, 1992.

von Zahn, U., and V. I. Moroz, Composition of the Venus atmosphere below $100 \mathrm{~km}$ altitude, Adv. Space Res., 5(11), 173-195, 1985. von Zahn, U., K. H. Fricke, D. M. Hunten, D. Krankowsky, K. Mauersberger, and A. O. Nier, The upper atmosphere of Venus during morning conditions, J. Geophys. Res., $85,768-770,1980$.

von Zahn, U., S. Kumar, H. Niemann, and R. Prinn, Composition of the Venus Atmosphere, in Venus, edited by D. M. Hunten et al., pp. 299-430, Univ. of Ariz. Press, Tucson, 1983.

Wiese, W. L., M. W. Smith, and B. M. Glennon, Atomic Transition Probabilities, Ref. Data Ser. Natl. Bur. Stand., U.S., 4, 1966.

Woo, R., and A. J. Kliore, Magnetization of the ionosphere of Venus and Mars: Results from radio occultation measurements, J. Geophys. Res., 96, 11,073-11,081, 1991.

Yoshino, K., J. R. Esmond, A. S.-C. Cheung, D. E. Freeman, and W. H. Parkinson, High resolution absorption cross sections in the transmission window region of the Schumann-Runge bands and Herzberg continuum of $\mathrm{O}_{2}$, Planet. Space Sci., 40, 185-192, 1992.

Young, R. A., and O. J. Dunn, Excitation and quenching of $\mathrm{N}\left({ }^{2} P\right)$, J. Chem. Phys., 63, 1150-1153, 1975.

Yung, Y. L., and W. B. DeMore, Photochemistry of the stratosphere of Venus: Implications for atmospheric evolution, Icarus, 51, 199-247, 1982.

Zipf, E. C., The dissociative recombination of vibrationally excited $\mathrm{N}_{2}^{+}$ions, Geophys. Res. Lett., 7, 645-648, 1980.

J. L. Fox, Department of Physics, Wright State University, Dayton, OH 45435, USA. (fox@platmo.phy.wright.edu)

K. Y. Sung, Marine Sciences Research Center, State University of New York at Stony Brook, Stony Brook, NY 11794-5000, USA. (ksung@atmsci.msrc.sunysb.edu)

(Received March 5, 2001; revised May 7, 2001; accepted May 7, 2001.) 